\title{
Improving conservative management of intermittent claudication
}

Citation for published version (APA):

Lauret, G. J. (2015). Improving conservative management of intermittent claudication. [Doctoral Thesis, Maastricht University]. Maastricht University. https://doi.org/10.26481/dis.20150708gl

Document status and date:

Published: 01/01/2015

DOI:

10.26481/dis.20150708gl

Document Version:

Publisher's PDF, also known as Version of record

\section{Please check the document version of this publication:}

- A submitted manuscript is the version of the article upon submission and before peer-review. There can be important differences between the submitted version and the official published version of record.

People interested in the research are advised to contact the author for the final version of the publication, or visit the DOI to the publisher's website.

- The final author version and the galley proof are versions of the publication after peer review.

- The final published version features the final layout of the paper including the volume, issue and page numbers.

Link to publication

\footnotetext{
General rights rights.

- You may freely distribute the URL identifying the publication in the public portal. please follow below link for the End User Agreement:

www.umlib.nl/taverne-license

Take down policy

If you believe that this document breaches copyright please contact us at:

repository@maastrichtuniversity.nl

providing details and we will investigate your claim.
}

Copyright and moral rights for the publications made accessible in the public portal are retained by the authors and/or other copyright owners and it is a condition of accessing publications that users recognise and abide by the legal requirements associated with these

- Users may download and print one copy of any publication from the public portal for the purpose of private study or research.

- You may not further distribute the material or use it for any profit-making activity or commercial gain

If the publication is distributed under the terms of Article $25 \mathrm{fa}$ of the Dutch Copyright Act, indicated by the "Taverne" license above, 


\section{IMPROVING CONSERVATIVE MANAGEMENT OF INTERMITTENT CLAUDICATION}

GERT-JAN LAURET 
Improving conservative management of intermittent claudication Thesis, University Maastricht, The Netherlands

Author: G.J. Lauret

ISBN:

Cover design: Indrek Viiderfeld, Tabasco Rock'n'Roll Ad Agency, Estonia (reprinted with permission)

Layout \& print: Ridderprint, Ridderkerk, the Netherlands

(c) 2015 G.J. Lauret, Eindhoven, The Netherlands

All rights reserved. No part of this publication may be printed or utilised in any form without permission of the copyright holder. 


\section{IMPROVING CONSERVATIVE MANAGEMENT OF INTERMITTENT CLAUDICATION}

\section{PROEFSCHRIFT}

ter verkrijging van de graad van doctor

aan de Universiteit Maastricht, op gezag van Rector Magnificus, prof. dr. L.L.G. Soete volgens het besluit van het College van Decanen, in het openbaar te verdedigen op woensdag 8 juli 2015 om 16.00 uur

door

Gert-Jan Lauret geboren te Brugge op 15 juli 1984 


\section{Promotoren}

Prof. dr. J.A.W. Teijink

Prof. dr. R.A. de Bie

\section{Copromotor}

Mw. dr. B.L.W. Bendermacher (Canisius-Wilhelmina Ziekenhuis, Nijmegen)

\section{Beoordelingscommissie}

Prof. dr. M.H. Prins (voorzitter)

Prof. dr. P.R.G. Brink

Dr. M.R.M. Scheltinga (Maxima Medisch Centrum, Veldhoven)

Mw. dr. E.M. Willigendael (Medisch Spectrum Twente, Enschede)

Financial support by the Dutch Heart Foundation for the publication of this thesis is gratefully acknowledged.

Additional financial support was generously provided by; Catharina Ziekenhuis Eindhoven, Stichting ClaudicatioNet, Fonds voor het Hart, Koninklijk Nederlandse Genootschap voor Fysiotherapie, W.L. Gore \& Associates, Rabobank EindhovenVeldhoven, McRoberts B.V., Krijnen Medical Innovations and Covidien.
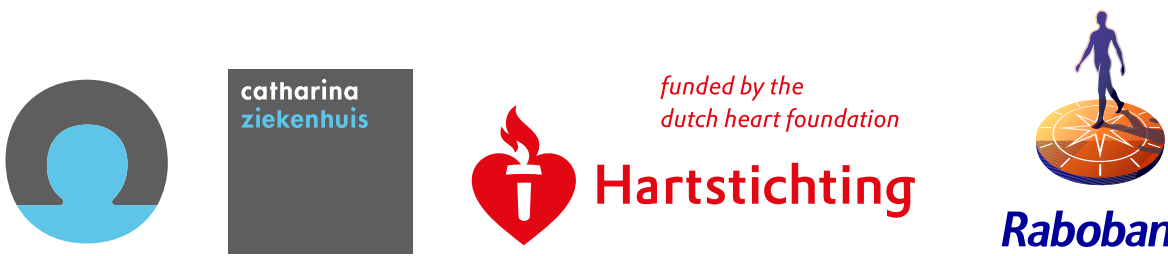

\section{Rabobank}

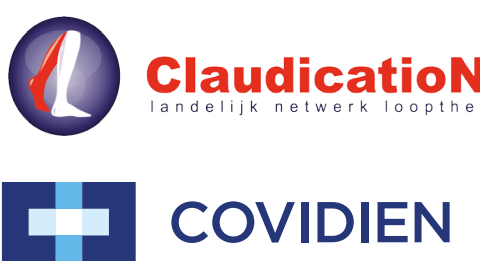

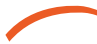

Fonds voor

het Hart

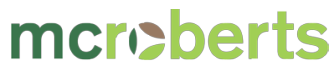


Voor mijn ouders 


\section{CONTENT OF THIS THESIS}

\section{CHAPTER 1}

INTRODUCTION AND OUTLINE OF THE THESIS

CHAPTER 2

SUPERVISED EXERCISE THERAPY:

CURRENT STATUS AND FUTURE PERSPECTIVES

CHAPTER 3

WHEN IS SUPERVISED EXERCISE THERAPY INDICATED FOR INTERMITTENT CLAUDICATION?

CHAPTER 4

MODES OF EXERCISE TRAINING

FOR INTERMITTENT CLAUDICATION

\section{CHAPTER 5}

PHYSICAL ACTIVITY MONITORING IN PATIENTS

WITH INTERMITTENT CLAUDICATION

\section{CHAPTER 6}

THE EFFECT OF SUPERVISED EXERCISE THERAPY ON PHYSICAL ACTIVITY AND AMBULATORY ACTIVITIES IN PATIENTS WITH INTERMITTENT CLAUDICATION 


\section{CHAPTER 7}

THE CLAUDICATIONET CONCEPT:

DESIGN OF A NATIONAL INTEGRATED CARE NETWORK FOR INTERMITTENT CLAUDICATION

CHAPTER 8

DEVELOPMENT OF QUALITY INDICATORS FOR

PHYSICAL THERAPY IN PERIPHERAL ARTERIAL

OCCLUSIVE DISEASE

CHAPTER 9

GENERAL DISCUSSION

CHAPTER 10

SUMMARY

SAMENVATTING

VALORISATIE

DANKWOORD

175

CURRICULUM VITAE

LIST OF PUBLICATIONS 



\section{CHAPTER 1}

\section{INTRODUCTION AND \\ OUTLINE OF THE THESIS}





\section{PERIPHERAL ARTERIAL OCCLUSIVE DISEASE}

Peripheral arterial occlusive disease (PAD) is a common condition that occurs due to a decrease in blood flow and oxygenation of tissue.' PAD mainly affects the lower extremities, with clinical manifestations ranging from no symptoms to intermittent claudication (IC) and possibly even tissue loss, which may eventually require amputation of the affected limb. ${ }^{2}$

$I C$ is the mildest clinical manifestation of PAD caused by progressive narrowing of the arteries of the peripheral circulation. IC manifests as a cramping pain in muscles of the calf, thigh, and/or buttock due to ischemia caused by insufficient blood flow and tissue oxygenation. Classically, IC manifests after a period of exercise and then subsides rapidly while resting. If PAD progresses to critical limb ischemia, tissue ischemia is even present during rest and a final stage involving tissue loss can occur.

\section{Etiology}

PAD is caused by the continuous process of atherosclerosis. Atherosclerosis can be defined as the progressive narrowing and hardening of arteries due to the accumulation of lipids, inflammatory cells, and smooth muscle cells within the intima of the vascular wall, forming an atherosclerotic plaque. ${ }^{3}$ The most predominant risk factors for atherosclerosis are smoking, hypertension, diabetes mellitus, hypercholesterolemia, and an increasing age. Although the process of atherosclerosis can occur in every artery, it can become symptomatic if it affects coronary, cerebral, abdominal and peripheral arteries of upper or lower extremities. Acute manifestations, like a heart attack or acute critical limb ischemia, are thought to occur when an atherosclerotic plaque ruptures, and the thrombogenic content of the plaque comes into contact with coagulation factors, causing the formation of a thrombus, which can occlude the artery. If the atherosclerotic plaque does not rupture, but is slowly progressive in thickness, chronic manifestations such as angina pectoris or PAD can occur due to a decrease in blood flow and oxygenation of tissue.

\section{Epidemiology}

Total disease prevalence of PAD, based on objective testing, has been evaluated in several epidemiologic studies and ranges from $3 \%$ to $10 \%$ in adults, increasing to $15 \%$ to $20 \%$ in persons over 70 years of age. ${ }^{4}$ Prevalence of $\mathrm{IC}$ is more difficult to determine, since the symptoms do not always predict the presence or absence of PAD. A patient with severe PAD may not show any symptoms of $I C$, because the patient is not physically active or the range of exercise is limited by some other condition. In contrast, some patients with symptoms suggesting IC may not have PAD; for instance spinal stenosis 
can produce symptoms similar to IC in the absence of vascular disease. Likewise, patients with very mild PAD may develop symptoms of IC only when they become very physically active. However, disease prevalence of $I C$ is estimated $3 \%$ in patients aged 40 and $6 \%$ in patients aged 60 years, contributing $15-40 \%$ to the PAD population. ${ }^{2}$ Although for IC patients the prognosis is favorable, with regard to PAD progression (and eventually amputation), they score considerably worse for many measures of quality of life and functional capacity measures than individuals without PAD. ${ }^{2,5,6}$ Furthermore, for IC, a 5-year mortality rate of $19 \%$ is described, $70 \%$ of which is due to cardiovascular causes. Non-fatal cardiovascular events (e.g. myocardial infarction or stroke) of patients with IC are found in $29 \%$ at 5 -year follow-up. ${ }^{7}$

\section{MANAGEMENT OF INTERMITTENT CLAUDICATION}

Treatment of IC (and PAD in general) should address two important targets: reduction of cardio- and cerebrovascular events (and associated morbidity and mortality) and improvement of health-related quality of life and functional capacity. To manage these targets, all patients with IC should receive cardiovascular risk management.

Cardiovascular risk management consists of antiplatelet therapy, statin therapy, treatment of hypertension, tight glycemic control, and lifestyle interventions aimed at smoking cessation, weight reduction and increase of physical activity. Limited data exists on lifestyle interventions in PAD. However, in coronary heart disease, lifestyle modification programmes were associated with reductions in all-cause mortality, cardiac mortality, cardiac readmissions and non-fatal re-infarctions. Furthermore, lifestyle interventions positively affected risk factors and related lifestyle behaviors at post-treatment and persisted at long-term follow up. ${ }^{8}$ Besides cardiovascular risk management, the primary and most effective treatment for IC is exercise therapy. ${ }^{9}$ The combination of both is considered the most important factor in improving associated health traits. ${ }^{10,11}$

\section{Exercise therapy}

In 1898, the successful results of exercise therapy for IC were first described by Wilhelm Erb, a German neurologist. ${ }^{12} \mathrm{He}$ described the amount of exercise necessary to provoke symptoms, localised the anatomical defect and described the societal implications of PAD. The first randomised controlled trial was performed by Larsen and Lassen in 1966. ${ }^{13}$ In this study, seven patients treated with exercise therapy were compared with a control group of seven patients who were given 'medical treatment' in the form of lactose tablets. The exercise group showed a significant increase in maximum walking time, whereas the patients in the control group did not improve. 
Nowadays, exercise therapy for patients with IC is extensively studied. ${ }^{14}$ In a Cochrane systematic review of randomized clinical trials, exercise therapy is associated with an overall improvement in walking ability of approximately $50 \%$ to $200 \%$ compared with no exercise therapy. Given its clear benefits, the importance of exercise therapy is highlighted in contemporary international guidelines. ${ }^{2,15}$

Exercise training provides significant symptomatic benefit for patients with IC; however, the exact mechanisms of this improvement remain unclear. ${ }^{16}$ The mechanisms of response to exercise training have been reviewed previously and include improvement in walking efficiency, induction of vascular angiogenesis, inflammatory activation, increased exercise pain tolerance, reversed endothelial and mitochondrial dysfunction, and metabolic adaptations within skeletal muscle. 2,17-20

\section{Supervised exercise therapy}

In contrast to exercise therapy, supervised exercise therapy (SET) entails adequate coaching by a physical therapist or another exercise specialist (e.g. an exercise physiologist, an exercise therapist, a human movement scientist or a specialized cardiovascular nurse). It aims to increase maximum walking distance, physical activity and health-related quality of life.

In a Cochrane systematic review, SET showed a significant improvement in maximal treadmill walking distance compared with non-supervised exercise therapy regimens, with an increase in walking distance of approximately 180 meters more in the supervised group. ${ }^{21}$ Although no clear evidence exists, most frequently described programs employ treadmill walking of sufficient intensity to cause claudication symptoms. Exercise is continued till near maximum pain, followed by rest, and then a next cycle of exercise is started over the course of a 30-60 minute session. During the exercise session, treadmill exercise is performed at a speed and grade that will induce claudication symptoms. The patient should stop walking when claudication pain is considered moderate. Exercise sessions are typically conducted three times a week for at least 3 months. 2,22

\section{Alternative exercise therapy}

Besides walking, there are alternative modes of SET, such as cycling, upper-extremity cycle ergometer exercise, and strength training, and these are also associated with a significantly improved walking capacity. ${ }^{23-25} \mathrm{~A}$ recent systematic review comparing any mode of exercise, whether supervised or unsupervised, was published. ${ }^{26}$ It suggested that alternative modes of aerobic exercise, other than walking, appear equally beneficial as walking exercise, while the effects of progressive resistance training and upper body exercise seem only promising. The effect size of each exercise mode was calculated 
and compared to the effect size of walking exercise. However, due to heterogeneity, no meta-analysis was performed on specific randomised controlled trials (RCTs) comparing the standard of supervised walking exercise to alternative exercise regimes.

\section{Home-based exercise therapy}

Although SET proved effective in the treatment of PAD by increasing maximal and pain-free walking time or distance, the existing exercise programs are not designed to change day-to-day walking behavior. ${ }^{27}$ Moreover, limitations continue to hinder implementation of supervised exercise programs in daily practice. Some describe a lack of a supervised setting to which patients can be referred or a lack of knowledge among referring healthcare professionals. In other studies participants were not willing to persist with an exercise program to maintain the benefit. $2,20,28$ Home-based walking programs that use a group-mediated cognitive behavioral intervention, incorporating both group support and self-regulatory skills, were chosen to be a solution for these patients. A home-based walking exercise program significantly improved walking endurance, physical activity, and patient-perceived walking endurance in patients with IC. ${ }^{28}$ These findings have implications for the large number of patients with PAD who are unable or unwilling to participate in supervised exercise programs.

\section{Physical activity monitoring}

Daily physical activity (PA) levels of IC patients are lower than those of healthy controls. ${ }^{29,30}$ However, PA is scantly used as an outcome parameter in trials concerning IC treatment. ${ }^{27,31}$ Although SET has proven effective in improving walking distances, it is questionable whether an increased walking capacity is of benefit during the patient's daily life activities. In other words, if an IC patient is able to walk further, this finding does not necessarily imply that PA levels and/or exercise behavior are positively influenced. Perhaps a potential goal of future PAD treatment should be to at increase the daily PA level.

In patients with IC, PA is rarely measured objectively in terms of energy expenditure. ${ }^{30,32,33}$ Regularly described methods for PA measurements are PA questionnaires, pedometers, or activity monitors. However, only the latter method is found capable of adequately assessing energy expenditure. ${ }^{34,35}$ The newest generation of activity monitors is based on tri-axial accelerometer techniques, measuring accelerations in three dimensions that can be converted to intensities and METs (metabolic equivalents). In this way, PA is determined more precisely, as was demonstrated by high correlations between indirect calorimetry and generated MET output at different walking speeds. ${ }^{34,36,37}$ 


\section{Invasive vascular interventions}

There are many surgical and endovascular interventions that can be used for the symptomatic treatment of IC, such as endarterectomy bypass surgery or percutaneous transluminal angioplasty (PTA), with or without stent placement. The general consensus is that patients with critical limb ischemia should undergo invasive vascular interventions to restore blood flow and to prevent amputation. ${ }^{2}$ However, the role of vascular interventions in IC is still debated. The preferred choice for primary treatment is often conservative management of SET and vascular risk management, since this is a safe and effective method and limb prognosis is favorable. This in contrast to the associated comorbidity and mortality of vascular interventions, estimated to be $<0.5 \%$ for PTA and up to $5-10 \%$ for bypass surgery. ${ }^{?}$

Nowadays, the effectiveness of management of IC is measured in most cases in terms of walking performances (using a treadmill protocol) or disease-specific quality of life questionnaires. In the last decade, several studies have compared SET, PTA and a combination of treatments for IC. In general, most studies included both patients with femoropopliteal and aortoiliac artery disease and found no difference between SET and PTA regarding walking distances or disease specific quality of life, even after 7 years of follow-up. ${ }^{38-43}$ Invasive interventions and reinterventions occurred more often in the PTA group. ${ }^{41}$ In a systematic review, SET was compared to PTA for all specific disease locations. ${ }^{43}$ Unfortunately, the investigators could not perform a meta-analysis due to shortcomings in the design of individual studies, the heterogeneity of interventions and the heterogeneity of assessment and reporting of outcome measures.

Results of individual RCTs lead to debate on how to manage patients with IC related to specific disease locations. For IC due to femoropopliteal disease, PTA and SET are found to be equally effective in improving walking distance and quality of life at a follow up of 12 months. ${ }^{42}$ For aortoiliac disease, SET resulted in a superior treadmill walking distance after 6 months compared to PTA, but PTA resulted in a non-significant greater improvement of disease-specific quality of life..$^{40}$ However, when combining both treatments, significantly better results in walking distance and disease specific quality of life are found for mixed aortoiliac and femoropopliteal disease, indicating a possible adjuvant benefit of combining both treatments. ${ }^{44-46}$

Choosing the optimal intervention for patients with IC can also be influenced decisively by the costs of interventions. In several cost-effectiveness studies, SET was considered the most cost-effective treatment for IC. ${ }^{47,48}$ However, in an economic analysis following a randomized controlled trial, a combination of SET and PTA appeared to be more 
cost-effective than PTA alone, since costs per quality adjusted life year (QALY) were significantly higher in the PTA group ( 11.777 for PTA vs. 10.650 for PTA + SET vs. 6.147 for SET). ${ }^{48}$

However, though proven to be effective, SET program are in many cases not fully reimbursed by healthcare insurance companies. As a consequence, SET is largely underutilized. Besides the reimbursement issues of SET, other arguments such as patient resistance and professional self-interest could play an additional role. ${ }^{49}$ Patients may receive an evidently insufficient and less cost-effective "go home and walk" advice, or a vascular intervention as an alternative first-line treatment strategy. A stepped care model strives to initially refer all IC patients to a SET program and reserve revascularization for those who do not respond to SET. Implementation of a stepped care model may lead to a significant annual saving of up to 33 million euros of healthcare resources. ${ }^{50}$

\section{AIM AND OUTLINE OF THE THESIS}

The aim of this thesis was to describe methods for improving management of IC in the Netherlands. This thesis elucidates possible indications for SET and presents a promising future outcome measure to evaluate SET. Furthermore, this thesis describes a novel care intervention to implement SET in the Netherlands. This thesis is important for both caregivers and healthcare policy makers, because it describes from a practical point of view when and how SET should be physically performed, implemented and evaluated using new outcome measures and quality indicators. From an organisational point of view, this thesis describes how a stepped-care conservative management of IC can be implemented in a chronic-care model for the Dutch population.

For patients with IC, SET is considered the cornerstone of conservative management, although cardiovascular management and lifestyle coaching are both considered equally important. In Chapter 2 the current knowledge and future perspectives on SET are described. Furthermore, we present the current evidence on cardiovascular risk management for IC patients. However, in clinical practice SET appears to be underutilized for different reasons. We therefore investigated the current opinion of Dutch vascular surgeons on SET as treatment option for PAD. We specifically investigated to what extent and on which criteria Dutch vascular surgeons decide to offer or deny SET to patients with IC. Results of this nationwide inquiry are described in Chapter 3. 
After elucidating the indications for SET in current clinical practice, this thesis focuses on the performance and clinical evaluation of SET programs. In Chapter 4, a systematic review describes the effects of different supervised exercise modes on maximal and pain-free walking distance. In Chapter 5, physical activity monitoring is used to describe a new outcome measure for evaluating SET, the real-life physical activity level. The daily physical activity level of patients with IC is determined and results are compared both with healthy adults and with current PA standards. The effect of SET on the daily physical activity level and ambulatory activities are described in Chapter 6. In particular, we wanted to find out the effect of a SET program on the number of patients achieving the ACSM/AHA public health minimum recommendations for physical activity.

Still, several shortcomings prevent SET from being available for the Dutch population of IC patients. Chapter 7 describes the organisational aspects of the shortcomings in the Dutch healthcare system. To improve the availability of SET, we provide the design of a national integrated care network (ClaudicatioNet). Based on the chronic care model as a conceptual framework, ${ }^{51}$ regional care networks of general practitioners, vascular surgeons, central caregivers and physical therapists are initiated to enhance the quality, access, continuity, efficiency and costs of the Dutch healthcare system.

An important factor in evaluating conservative management of IC is how to measure its quality. At present, there is no evidence of how the quality of physical therapy management should be monitored. Therefore, in Chapter 8 quality indicators for SET are developed using an expert Delphi procedure.

In Chapter 9, the main findings of this thesis are discussed. Methodological aspects of the different studies, implications for clinical practice, and implications for future research are described. Finally in Chapter 10, this thesis is summarised both in English and in Dutch. 


\section{REFERENCES}

1. Golledge J. Lower-limb arterial disease. Lancet. 1997;350(9089):1459-1465.

2. Norgren L, Hiatt WR, Dormandy JA, Nehler MR, Harris KA, Fowkes FG. Inter-Society Consensus for the Management of Peripheral Arterial Disease (TASC II). J Vasc Surg. 2007;45(Suppl S):S5-67.

3. Lusis AJ. Atherosclerosis. Nature. 2000;407(6801):233-41.

4. Selvin E, Erlinger TP. Prevalence of and risk factors for peripheral arterial disease in the United States: results from the National Health and Nutrition Examination Survey, 1999-2000. Circulation. 2004;110(6):738-43.

5. Regensteiner JG, Hiatt WR, Coll JR, Criqui MH, Treat-Jacobson D, McDermottMM, Hirsch AT. The impact of peripheral arterial disease on healthrelated quality of life in the Peripheral Arterial Disease Awareness, Risk, and Treatment: New Resources for Survival (PARTNERS) Program. Vasc Med. 2008;13(1):15-24.

6. Dumville JC, Lee AJ, Smith FB, Fowkes FG The health-related quality of life of people with peripheral arterial disease in the community: the Edinburgh Artery Study. Br J Gen Pract. 2004;54(508):826-831.

7. Leng GC, Lee $A J$, Fowkes FG, Whiteman $M$, Dunbar J, Housley $E$, et al. Incidence, natural history and cardiovascular events in symptomatic and asymptomatic peripheral arterial disease in the general population. Int J Epidemiol. 1996;25(6):1172-81

8. Janssen V, De Gucht V, Dusseldorp E, Maes S. Lifestyle modification programmes for patients with coronary heart disease: a systematic review and meta-analysis of randomized controlled trials. Eur J Prev Cardiol. 2013;20(4):620-40

9. Stewart KJ, Hiatt WR, Regensteiner JG, Hirsch AT. Exercise training for claudication. New Eng J Med. 2002;347(24):1941-51.

10. Leeper NJ, Myers J, Zhou M, Nead KT, Syed A, Kojima $Y$, et al. Exercise capacity is the strongest predictor of mortality in patients with peripheral arterial disease. J Vasc Surg. 2013;57(3):72833.

11. Gardner AW, Montgomery PS, Parker $D E$. Physical activity is a predictor of allcause mortality in patients with intermittent claudication. J Vasc Surg. 2008;47(1):1 17-22.
12. Erb W. About intermittent walking and nerve disturbances due to vascular disease. Deutsch Z Nervenheilk.1898;13:1-76.

13. Larsen OA, Lassen NA. Effect of daily muscular exercise in patients with intermittent claudication. Lancet. 1966;2:1093-6.

14. Watson L, Ellis B, Leng GC. Exercise for intermittent claudication. Cochrane Database Syst Rev. 2008;4:CD000990

15. Hirsch AT, Haskal Z, Hertzer NR, Bakal CW, Creager MA, Halperin JL, et al. ACC/AHA 2005 Practice Guidelines for the management of patients with peripheral arterial disease. Circulation. 2006;1 13(11):e463-654.

16. Beckitt TA, Day J, Morgan M, Lamont PM Calf muscle oxygen saturation and the effects of supervised exercise training for intermittent claudication. J Vasc Surg. 2012;56(2):470-5.

17. Gustafsson T, Kraus WE. Exercise-induced angiogenesis related growth and transcription factors in skeletal muscle, and their modification in muscle pathology. Front Biosci. 2001;1(6):D75-89.

18. Hamburg NM, Balady GJ. Exercise rehabilitation in peripheral artery disease: functional impact and mechanisms of benefits. Circulation. 2011;123(1):87-97.

19. Zwierska I, Walker RD, Choksy SA, Male JS, Pockley AG, Saxton JM. Upper- vs lowerlimb aerobic exercise rehabilitation in patients with symptomatic peripheral arterial disease: a randomized controlled trial. J Vasc Surg. 2005;42(6): 1 122-30.

20. Stewart AH, Smith FC, Baird RN, Lamont PM. Local versus systemic mechanisms underlying supervised exercise training for intermittent claudication. Vasc Endovasc Surg. 2008;42(4):314-20.

21. Fokkenrood $\mathrm{HJ}$, Bendermacher $\mathrm{BL}$, Lauret GJ, Willigendael EM, Prins $M H$, Teijink JA. Supervised exercise therapy versus nonsupervised exercise therapy for intermittent claudication. Cochrane Database Syst Rev. 2013;8;CD005263.

22. Merry AH, Teijink JA, Jongert MW, Poelgeest A, van der Voort SS, Bartelink ME. et al. KNGF Richtliin Symptomatisch Perifeer Arterieel Vaatlijden. The Royal Dutch Society for Physical Therapy. www.fysionet.nl 
23. Hiatt WR, Wolfel EE, Meier RH, Regensteiner JG. Superiority of treadmill walking exercise versus strength training for patients with peripheral arterial disease. Implications for the mechanism of the training response. Circulation. 1994;90(4): 1866-74.

24. Sanderson B, Askew C, Stewart I, Walker P, Gibbs H, Green S. Short-term effects of cycle and treadmill training on exercise tolerance in peripheral arterial disease. J Vasc Surg. 2006;44(1): 119-27.

25. Treat-Jacobson D, Bronas UG, Leon AS. Efficacy of armergometry versus treadmill exercise training to improve walking distance in patients with claudication. Vasc Med. 2009;14(3):20313.

26. Parmenter BJ, Raymond J, Dinnen P, Singh MA. A systematic review of randomized controlled trials: walking versus alternative exercise prescription as treatment for intermittent claudication. Atherosclerosis. 2011;218(1):112.

27. Crowther RG, Spinks WL, Leicht AS, Sangla K, Quigley F, Golledge J. Effects of a longterm exercise program on lower limb mobility, physiological responses, walking performance, and physical activity levels in patients with peripheral arterial disease. J Vasc Surg. 2008;47(2):303-9.

28. Makris GC, Lattimer CR, Lavida A, Geroulakos $G$. Availability of supervised exercise programs and the role of structured home-based exercise in peripheral arterial disease. Eur J Vasc Endovasc Surg. 2012;44(6):569-75.

29. Gardner AW, Womack CJ, Sieminski DJ, Montgomery PS, Killewich LA, Fonong T. Relationship between free-living daily physical activity and ambulatory measures in older claudicants. Angiology. 1998;49:327-37.

30. Sieminski DJ, Gardner AW. The relationship between free-living daily physical activity and the severity of peripheral arterial occlusive disease. Vasc Med. 1997;2:286-91.

31. Gardner AW, Montgomery PS, Parker DE. Optimal exercise program length for patients with claudication. J Vasc Surg. 2012;55:134654.
32. McDermott MM, Liu K, O'Brien E, Guralnik $J M$, Criqui MH, Martin GJ, et al. Measuring physical activity in peripheral arterial disease: a comparison of two physical activity questionnaires with an accelerometer. Angiology. 2000;51(2):91-100.

33. Sieminski DJ, Cowell LL, Montgomery PS, Pillai SB, Gardner AW. Physical activity monitoring in patients with peripheral arterial occlusive disease. J Cardiopulm Rehabil. 1997;17(1):437.

34. van Hees VT, van Lummel RC, Westerterp KR. Estimating activity-related energy expenditure under sedentary conditions using a tri-axial seismic accelerometer. Obesity. 2009; 17(6):1287-92.

35. Gardner AW, Poehlman ET. Assessment of free-living daily physical activity in older claudicants: validation against the doubly labeled water technique. J Gerontol A Biol Sci Med Sci. 1998;53(4):275-80.

36. Van Remoortel H, Giavedoni S, Raste Y, Burtin C, Louvaris Z, Gimeno-Santos E, et al. Validity of activity monitors in health and chronic disease: a systematic review. Int J Behav Nutr Phys Act. 2012;9:84.

37. Van Hees VT, Slootmaker SM, De Groot G, Van Mechelen W, Van Lummel RC. Reproducibility of a triaxial seismic accelerometer (DynaPort). Med Sci Sports Exerc. 2009;41(4):810-7.

38. Spronk S, Bosch JL, den Hoed PT, Veen HF, Pattynama PM, Hunink MG. Intermittent claudication: clinical effectiveness of endovascular revascularization versus supervised hospital-based exercise training - randomized controlled trial. Radiology. 209;250(2):586-95.

39. Nordanstig J, Gelin J, Hensater M, Taft C, Osterberg K, Jivegard L. Walking performance and health-related quality of life after surgical or endovascular invasive versus non-invasive treatment for intermittent claudication - a prospective randomized trial. Eur J Vasc Endovasc Surg. $2011 ; 42(2): 220-7$.

40. Murphy TP, Cutlip DE, Regensteiner JG, Mohler ER, Cohen DJ, Reynolds MR et al. Supervised exercise versus primary stenting for claudication resulting from aortoiliac peripheral arterial disease: six-month outcomes from the claudication: exercise versus endoluminal (CLEVER) study. Circulation. 2012;125(1):1309. 
41. Fahkry F, Rouwet EV, den Hoed PT, Hunink MG Spronk $S$. Long-term clinical effectiveness of supervised exercise therapy versus endovascular revascularisation for intermittent claudication from a randomized clinical trial. $\mathrm{Br} J$ Surg. 2013;100(9):1164-71.

42. Mazari FA, Khan JA, Carradice D, Samuel $N$, Abdul Rahman MN, Gulati $S$, et al. Randomized clinical trial of percutaneous transluminal angioplasty, supervised exercise and combined treatment for intermittent claudication due to femoropopliteal disease. $\mathrm{Br}$ J Surg. 2012;99(1):39-48

43. Frans FA, Bipat $S$, Reekers JA, Legemate DA, Koelemay MJ. Systematic review of exercise training or percutaneous transluminal angioplasty for intermittent claudication. $\mathrm{Br} \mathrm{J}$ Surg. 2012;99(1):16-28

44. Kruidenier LM, Nicolai SP, Rouwet EV, Peters RJ, Prins MH, Teiijnk JA. Additional supervised exercise therapy after a percutaneous vascular intervention for peripheral arterial disease: a randomized clinical trial. J Vasc Interv Radiol. $2011 ; 22(7): 961-8$.

45. Greenhalgh RM, Belch JJ, Brown LC, Gaines PA, Gao L, Reise JA, et al. The adjuvant benefit of angioplasty in patients with mild to moderate intermittent claudication (MIMIC) managed by supervised exercise, smoking cessation advice and best medical therapy: results from two randomised trials for stenotic femoropopliteal and aortoiliac arterial disease. Eur J Vasc Endovasc Surg. 2008;36(6):680-8.
46. Bo E, Hisdal J, Cvancarova M, Stranden E, Jorgensen JJ, Sandbaek $G$, et al. Twelvemonths follow-up of supervised exercise after percutaneous transluminal angioplasty for intermittent claudication: a randomised clinical trial. Int J Environ Res Public Health. 2013;11(11):5998-6014.

47. Spronk S, Bosch JL, den Hoed PT, Veen HF, Pattynama PM, Hunink MG. Cost-effectiveness of endovascular revascularization compared to supervised hospital-based exercise training in patients with intermittent claudication: a randomized controlled trial. J Vasc Surg. 2008;48(6): 1472-80

48. Mazari FA, Khan JA, Carradice D, Samuel $\mathrm{N}$, Gohil R, McCollum PT, et al. Economic analysis of a randomized trial of percutaneous angioplasty, supervised exercise or combined treatment for intermittent claudication duet 0 femoropopliteal arterial disease. $\mathrm{Br} \mathrm{J}$ Surg. 2013;100(9):1172-9.

49. Popplewell MA, Bradbury AW. Why Do Health Systems Not Fund Supervised Exercise Programmes for Intermittent Claudication? Eur J Vasc Endovasc Surg. 2014;48(6):608-610.

50. Fokkenrood HJ, Scheltinga MR, Koelemay MJ, Breek JC, Hasaart F, Vahl AC, et al. Significant savings with a stepped care model for treatment of patients with intermittent claudication. Eur J Vasc Endovasc Surg. 2014;48(4):423-9.

51. Epping-Jordan JE, Pruitt SD, Bengoa R, Wagner $\mathrm{EH}$. Improving the quality of health care for chronic conditions. Qual Saf Health Care. 2004;13:299-305. 


\section{CHAPTER 2}

\section{SUPERVISED EXERCISE THERAPY: CURRENT STATUS AND FUTURE PERSPECTIVES}

G.J. Lauret

H.C.W. van Dalen

E.M. Willigendael

H.J.M. Hendriks

R.A. de Bie

S. Spronk

J.A.W. Teijink 


\section{ABSTRACT}

Intermittent claudication (IC) has a high prevalence in the older population and is closely associated with cardiovascular and cerebrovascular disease. High mortality rates are reported due to ongoing atherosclerotic disease. Because of these serious health risks, treatment of IC should address reduction of cardiovascular events (and related morbidity/mortality) and improvement of the poor health-related quality of life (QoL) and functional capacity. In several randomized clinical trials and systematic reviews, supervised exercise therapy (SET) is compared with non-supervised exercise, usual care, placebo, walking advice or vascular interventions. The current evidence supports SET as the primary treatment for IC. SET improves maximum walking distance and health-related QoL with a marginal risk of co-morbidity or mortality. This is also illustrated in contemporary international guidelines. Community-based SET appears to be at least as efficacious as programs provided in a clinical setting. In the Netherlands, a national integrated care network (ClaudicatioNet) providing specialized care for patients with IC is currently being implemented. Besides providing a standardized form of SET, the specialized physical therapists stimulate medication compliance and perform lifestyle coaching. Future research should focus on the influence of comorbidities on prognosis and effect of SET outcome and the potential beneficial effects of SET combined with a vascular intervention. 


\section{INTRODUCTION}

The atherosclerotic process of progressive narrowing and hardening of arteries can occur in every artery in the human body, however it mainly affects the coronary, cerebral and peripheral arteries of the lower extremities. ${ }^{1,2}$ Lower extremity peripheral arterial occlusive disease (PAOD) has an estimated prevalence between $1.6 \%$ to $4.5 \%$ in the general population and rises to $19.1 \%$ in the Dutch population, aged 55 years and older. ${ }^{3-6}$ The primary sites of involvement of PAOD are the femoral and popliteal arteries in $80-90 \%$, the tibial and peroneal arteries in $40-50 \%$ and in $30 \%$ the aorta and iliac arteries. ${ }^{7}$ The manifestation of PAOD ranges from no symptoms to tissue loss that eventually requires amputation of the affected limb. The majority of patients with PAOD, have asymptomatic or atypical disease. ${ }^{2}$

Intermittent claudication (IC), the mildest symptomatic manifestation of PAOD, has a prevalency in the general population of approximately $3 \%$ in patients aged 40 , to $6 \%$ in patients aged 60 years and increasing in older age. 2 In women, the incidence rates of IC are approximately $50 \%$ lower than in men. ${ }^{8,9}$ In IC, progressive atherosclerotic narrowing results in decreased oxygen availability in the periphery of the buttocks and/or lower extremities. During exercise, the lack of oxygen causes an anaerobic state in which lactic acid and other metabolites are formed. This causes cramping or aching pain in the buttock, hip, thigh, calf or in rare occasions the foot, forcing the patient to pause. In rest, the oxygen debt can be redeemed and symptoms are relieved. However, when PAOD progresses, the pain becomes constant and the increasing oxygen debt can no longer be redeemed. This condition, called critical limb ischemia, can eventually progress to the final stage of tissue loss. Although amputation is the greatest fear for patients with IC, the prognosis of the affected limb is favorable. After a 5 -year follow-up period, $28.8 \%$ of all patients with IC still have claudication symptoms and only $1.6 \%-4.1 \%$ need a limb amputation. ${ }^{8,9}$

PAOD and IC are strong predictors for coinciding atherosclerotic disease and related mortality. The prevalence of cerebrovascular disease in patients with IC is about $25-50 \% .^{10}$ The 10-year mortality rate due to cardiovascular disease is $62 \%$ for men with PAOD compared to $17 \%$ in the population of men without PAOD. For women with PAOD the 10year mortality rate is $33 \%$ compared to $12 \%$ without PAOD. ${ }^{11}$ In a subgroup of patients with severe and symptomatic PAOD a 15-fold increase in mortality rate was found. ${ }^{11}$ For IC, a 5-year mortality rate of $19.2 \%$ is described, of which $70 \%$ is due to cardiovascular causes. Non-fatal cardiovascular events (e.g. myocardial infarction, stroke) in patients with IC are found in $29 \%$ at 5 year of follow-up. ${ }^{9}$ Compared to 
patients with IC, subjects with asymptomatic PAOD appear to have the same increased risk of cardiovascular events and death. ${ }^{9}$ Nonetheless, for those afflicted, IC has a negative impact on walking ability and health-related quality of life (QoL). ${ }^{12,13}$

Because of these serious health risks, treatment of IC (and PAOD in general) should address two important targets; reduction of cardiovascular events (and related morbidity and mortality) and improvement of the poor health-related QoL and functional capacity. To manage these targets, all patients with IC should receive a multicomponent therapy consisting of cardiovascular risk modification including lifestyle coaching and supervised exercise therapy (SET). A (minimal) invasive vascular intervention should be performed only when indicated. Since there is no outcome measurement available that truly compares the benefit of different treatment strategies (i.e. SET vs. vascular interventions vs. a combination of both), clear criteria to identify patients that will benefit the most from a certain intervention are not available. Existing outcome measurements like walking distances, exercise tests, health related QoL questionnaires and arterial patency each have their flaws and are not ideally suitable to compare the contribution of the different treatments to the ultimate goal of treatment; regaining physical activity.

In this article we aim to provide an up to date overview of the current literature regarding SET as an integrated part of the treatment for patients with IC. In addition we will address some future perspectives and trends in the management of IC that are linked to this multicomponent strategy.

\section{CURRENT STATUS}

\section{General management of IC}

The development of IC is accelerated by the same cardiovascular risk factors as known for other expressions of atherosclerotic disease (i.e., coronary heart disease and cerebrovascular disease). Treatment of IC should therefore consist of a multicomponent therapy of cardiovascular risk modification (1), including lifestyle coaching (2) and symptomatic treatment (3). The first two components aim to prevent cardiovascular events (myocardial infarction, stroke) and related morbidity and mortality. Recommendations from numerous consensus documents, including the American College of Cardiology/ American Heart Association (ACC/AHA) and Trans-Atlantic Inter-Society Consensus on Management of Peripheral Arterial Disease (TASC II) guidelines on PAOD, identify patients with PAOD as a high-risk population who require intensive risk factor modification. ${ }^{1,2}$ The modifiable risk factors for atherosclerosis are smoking, hypertension, diabetes mellitus, hyperlipidemia and obesity. ${ }^{14}$ 
International guidelines support the aggressive treatment of high blood pressure in patients with PAOD. ${ }^{15,16}$ In this high-risk group the current recommendation is a goal of $140 / 90 \mathrm{mmHg}$, or even $130 / 80 \mathrm{mmHg}$ if the patient also has diabetes or renal insufficiency. In PAOD, thiazides and ACE inhibitors should be considered as initial blood-pressure lowering drugs to reduce the risk of cardiovascular events. Betaadrenergic blocking drugs have been discouraged in PAOD because of the possibility of worsening claudication symptoms. In a Cochrane review there was no supporting evidence that beta-blockers adversely affect walking distance in people with intermittent claudication. ${ }^{17}$ However, due to the lack of large published trials beta blockers should be used with caution, but are not contraindicated in PAOD.

Diabetes increases the risk of PAOD approximately three- to four-fold, and the risk of claudication two-fold. Diabetes is also associated with peripheral neuropathy, which could lead to an increased risk of foot ulcers and foot infections. In recent years, there has been much discussion about the optimal treatment strategy (aggressive or standard glucose lowering therapy) of type 2 diabetes mellitus. In a meta-analysis, an intensive glucose-lowering regime (glycated haemoglobin level below 6.0\%) was compared to standard therapy (targeted a level of 7.0-7.9\%) in type 2 diabetes mellitus. Overall, intensive therapy significantly reduced coronary events without a significant effect on events of stroke or all-cause mortality. ${ }^{18}$ However, aggressive control of blood glucose levels can not be recommended after publication of the long term results of intensive therapy. The results described a significantly reduced nonfatal myocardial infarction risk but an increased 5-year all-cause mortality rate (hazard ratio 1.21 ; $95 \%$ C.I. 1.021.44) related with aggressive glucose lowering. ${ }^{19}$

In case of hyperlipidemia, dietary modification should be the initial intervention to control abnormal lipid levels. The Heart Protection Study demonstrated the benefits of cholesterol-lowering statin therapy in 6.748 patients with PAD and 13.788 other highrisk participants, regardless of their presenting cholesterol levels. ${ }^{20}$ Allocation to 40 $\mathrm{mg}$ simvastatin daily reduced the rate of the first major vascular events by about onequarter, and that of peripheral vascular events by about one-sixth, with large absolute benefits seen in participants with PAD because of their high vascular risk. Consequently, according this study statin therapy should be considered for all patients with PAOD. In contrast, the older ACC/AHA guidelines recommend achieving an LDL cholesterol level $<2.59 \mathrm{mmol} / \mathrm{L}(<100 \mathrm{mg} / \mathrm{dL})$ in all patients with PAOD. In patients with PAOD and a history of other vascular disease (i.e., coronary heart disease and cerebrovascular disease) it is reasonable to lower LDL cholesterol levels to $1.81 \mathrm{mmol} / \mathrm{L}(70 \mathrm{mg} / \mathrm{dL}){ }^{1}$ Statins should be the primary lipid-lowering agents to lower LDL cholesterol levels. 
Blood homocysteine levels are positively associated with cardiovascular disease, but it is uncertain whether the association is causal. Studies, investigating the effects of folic acid supplementation on major vascular events in patients with peripheral arterial disease, are lacking. However, in a RCT, including 12.064 survivors of myocardial infarction, long-term reductions in blood homocysteine levels with folic acid and vitamin B12 supplementation did not lead to a reduction of cardiovascular events and seems therefore not indicated. ${ }^{21}$

Antiplatelet therapy reduces major vascular events (vascular death, nonfatal $\mathrm{MI}$ and nonfatal stroke) in patients with PAOD by $23 \% .{ }^{22}$ Therefore, all symptomatic patients with or without a history of other cardiovascular disease should be prescribed long term antiplatelet therapy. Unfortunately, adherence to cardiovascular medication is fairly low. ${ }^{23}$ Self-reported consistent use (reported on $\geq 2$ consecutive follow-up surveys and then through death, withdrawal, or study end) of cardiovascular medication was analysed using the Duke Databank for Cardiovascular Disease in patients with coronary artery disease, with or without heart failure. ${ }^{24}$ In 2002, consistent use was reported: for aspirin, $71 \%$; beta-blockers, $46 \%$; lipid-lowering therapy, $44 \%$; aspirin and beta-blockers, $36 \%$; and all three, $21 \%$. Because of these findings the assessment of medication compliance should be incorporated in the standard care for patients with PAOD.

Medication compliance as part of a SET program leans towards lifestyle coaching. Lifestyle coaching is the second component of the multicomponent therapy for IC and forms an essential part of cardiovascular risk modification. In general, the existence and potential value of lifestyle coaching programmes are well known. However, in most cases lifestyle coaching forms no part of standard care for patients with PAOD. Essential topics of lifestyle coaching are smoking cessation, dietary advice, weight control and stimulation of physical activity. Smoking is considered the single most important modifiable risk factor for the development of PAOD. Smokers have a risk of PAOD that is 4 times that of non-smokers and experience onset of symptoms almost one decade earlier. ${ }^{24-26}$ However, patients who are able to quit smoking are less likely to develop critical limb ischemia and have improved survival. ${ }^{27}$

Patients who are moderately overweight (body mass index 25-30) or who are obese (body mass index $>30$ ) should receive counselling for weight reduction by means of dietary advice and stimulation of physical activity. Dietary advice should address the need for a negative caloric balance with carbohydrate restriction and reduction of calorie intake. The level of physical activity should be assessed and compared to 
the global recommendations on physical activity for health, composed by the World Health Organization. ${ }^{28}$ All adults should do at least 150 minutes or moderate-intensity aerobic physical activity (usually a 5 or 6 on a scale of 0-10 of personal capacity) or 75 minutes of vigorous-intensity aerobic physical activity (usually a 7 or 8 on a scale of 0-10 of personal capacity) throughout the week.

The third and last component addresses the poor health-related QoL in patients with IC. Patients with IC have a considerably poorer health status, functional capacity and health-related QoL compared to individuals without PAOD. ${ }^{12,13}$ Treatments used to improve these impairments are pharmacologic treatment, (supervised) exercise therapy and invasive forms of treatment (e.g. percutaneous and surgical vascular interventions). The latter are occasionally used as first-line treatment.

Pharmacologic treatment for relief of claudication symptoms typically involves other drugs than those used for risk reduction. Cilostazol is currently the most effective drug for IC. ${ }^{29}$ Approved by the FDA in 1999, the primary action of cilostazol is to inhibit phosphodiesterase type 3 , which results in vasodilatation and inhibition of platelet aggregation, arterial thrombosis and vascular smooth muscle proliferation. A three to six-month course of cilostazol is a possible first line pharmacotherapy for the relief of claudication symptoms, as evidence shows both an improved walking distance as well as QoL. ${ }^{30}$ Naftidrofuryl can also be considered. In a meta-analysis naftidrofuryl showed an clinically meaningful improvement in pain-free and maximum walking distance in patients with IC. ${ }^{31}$ It is a 5-hydroxytryptamine type 2 antagonist and may improve muscle metabolism, and reduce erythrocyte and platelet aggregation. Approval of cilostazol or naftidrofuryl for IC is however limited to certain countries.

\section{Exercise Therapy}

Exercise therapy is the first suggested therapy for patients with IC. In 1898 Wilhelm Erb, a German neurologist, described the successful results of exercise therapy for a patient with IC. ${ }^{32}$ The first randomised clinical trial (RCT) was performed by Larsen en Lassen in $1966 .{ }^{33}$ In this study, 7 patients treated with exercise therapy were compared with a control group of 7 patients who were given 'medical treatment' in the form of lactose tablets. For the group treated with exercise, a significant increase in maximum walking time was seen, whereas the patients in the control group did not improve.

Nowadays, exercise therapy for patients with IC is extensively studied. In a Cochrane review by Watson et al. exercise therapy was compared with usual care or placebo on data of functional capacity outcome measurements. ${ }^{34} \mathrm{~A}$ total of twenty-two trials met the 
inclusion criteria involving a total of 1200 participants with IC. In conclusion, compared to placebo and usual care, exercise therapy significantly improved maximal walking time with a mean difference of 5.12 minutes (95\% confidence interval $4.51-5.72$ ) and an improved maximum walking distance of 113.2 metres (range 95.0 to 131.4). Exercise therapy also showed a positive effect on the reduction of cardiovascular risk factors including hypercholesterolemia, hypertension, and diabetes mellitus. ${ }^{35}$

The most common exercise therapy prescription consists of a single oral advice, usually without supervision or follow-up. The adherence of patients given an oral exercise advice appears to be low. Comorbidity, lack of (specific) advice, fear, and lack of discipline and supervision are barriers to actually perform regular walking exercise. ${ }^{36}$ For these reasons the importance of supervision was recognised.

\section{Supervised exercise therapy}

Supervised exercise therapy (SET) entails adequate coaching by a physical therapist (PT) or other exercise specialist (e.g. exercise physiologist, exercise therapist, specialised cardiovascular nurse) and aims to increase maximal walking distance, physical activity and health-related QoL. The most effective programs employ treadmill walking that is of sufficient intensity to bring on claudication symptoms. Exercise is continued till near maximum pain, followed by rest, over the course of a 30-60 minute session. Exercise sessions are typically conducted three times a week for 3 months. ${ }^{2}$ A Cochrane review by Bendermacher et al. compared SET with non-supervised exercise programmes for patients with IC. ${ }^{37}$ SET showed statistically significant and clinically relevant differences in improvement of maximal walking distance compared with non-supervised exercise therapy regimens, with an overall effect size of 0.58 (95\% confidence interval 0.31 to 0.85 ) at three months. This translates into an improvement of approximately 150 meters of maximum walking distance in favour of the supervised group. However, additional studies on QoL appear to be needed to definitely demonstrate clinical effectiveness.

In a number of RCT's SET is compared to endovascular revascularisation or surgical reconstruction. Spronk et al. compared functional capacity and health-related QoL in patients with IC during a 12 month follow-up after endovascular revascularisation or hospital-based SET. ${ }^{38}$ The results demonstrate that after 12 months patients with IC benefited equally from either endovascular revascularisation or hospital-based SET. Improvement is however more instantly following revascularisation. In another RCT, Creasy et al. found a progressive and significant improvement in walking distance for patients treated with exercise therapy. For the group treated with endovascular revascularisation a non-significant increase in walking distance was found after 3 
months followed by a steady detoriation until 12 months follow-up. ${ }^{39}$ Only one study compared SET to surgical reconstruction. ${ }^{40}$ In both groups walking distance improved but no significant difference was found.

To evaluate the complementary effects of angioplasty with SET, Mazari et al. randomised claudicants with angioplasty-suitable femoropopliteal lesions, to SET, angioplasty or angioplasty + SET. All the groups demonstrated significant clinical and quality of life improvements, but the angioplasty + SET group produced a much greater improvement in clinical outcome measures than angioplasty or SEP alone, without a significant QoL improvement. ${ }^{41}$ Also, in the MIMIC trial patients with femoropopliteal and aortoiliac arterial disease, were randomised to receive either PTA or no PTA against a background of supervised exercise and best medical therapy. The maximum walking distance was $38 \%$ greater in the PTA group for the femoropopliteal trial $(95 \% ; \mathrm{Cl} 1-90)(\mathrm{p}=0.04)$ and $78 \%$ greater in the PTA group for the aortoiliac trial $(95 \% ; \mathrm{Cl} 0-216)(p=0.05)$. No benefits were found for health-related QoL.42

Few studies consider the long-term (>12 months) effects of SET. Gardner et al. tried to determine whether improvements in physical function after 6 months of SET could be sustained over a subsequent 12-months in older patients with IC. ${ }^{43}$ They concluded that improvements in maximum walking distance and physical activity level, after 6 months of exercise training, are prolonged for an additional 12 months using a less intense exercise maintenance program. Ratliff et al. reported a 3-year follow-up of 212 patients with IC who initially were treated with SET, with an exercise programme of two sessions a week for 10 weeks. ${ }^{44}$ Their results show that the maximum walking distance seen at 12 weeks is still present at three years. Based on this limited experience, it appears that SET has long-term benefits for patients with IC.

In the majority of all reviewed studies on SET, an outpatient hospital setting was offered. This approach seems appropriate in trials, but has several limitations in daily clinical practise. First, the capacity of an exercise therapy program in an outpatient clinic is limited and not sufficient to provide SET for all patients with IC. Second, attending a hospital 3 times a week comes with considerable transportation costs and is timeconsuming for the individual patient. For this reason, implementation of a communitybased SET program was instigated. ${ }^{45}$ The first results of a cohort study of patients treated with community-based SET resulted in a highly statistically significant improvement in maximum walking distance (on a treadmill) after 3 and 6 months. ${ }^{46}$ The authors concluded that comparison of these results with historical studies on hospital-based SET should be done with caution due to the variability in the prescribed exercise regimens 
and used treadmill walking tests. However, SET in a community-based setting seemed to be at least as efficacious as the programs provided in a clinical setting, but with a higher capacity.

In the ExitPAD trial, a multicenter RCT, Nicolai et al. compared exercise therapy in the form of a 'go home and walk' advice (WA) with community-based SET for patients with IC. ${ }^{47}$ SET appeared to be significantly more effective than WA in improving maximum walking distance and health-related QoL. The data from the ExitPAD trial were also used to assess the cost-effectiveness of SET versus WA. ${ }^{48}$ For community-based SET, the incremental cost-effectiveness ratio for cost per QALY was 28.693. At a willingness-topay threshold of 40.000 per QALY, SET seemed a cost-effective therapeutic option for patients with IC. Based on this evidence, (community-based) SET programs should be made available for all patients with IC, as stated in several national and international guidelines. 1 2, 49, 50

\section{FUTURE PERSPECTIVES}

Although SET is considered the best evidence based therapy for all patients with IC, general practitioners, vascular surgeons or vascular specialists do not always have the disposal of (community-based) physical or exercise therapists (PTs) with specific knowledge of IC or exercise training. Also, not all PTs have sufficient experience with this specific patient category. Patients suffer from a variety of comorbidities and modifiable lifestyle factors, potentially generating suboptimal results. Unfortunately, too many examples of PTs treating patients with IC with massages and other alternative, non-evidence based treatments exist. For this reason Nicolaï et al extensively trained all participating community-based PTs before they were allowed to participate in the ExitPAD trial. ${ }^{47}$

To standardise treatment and assure quality of PTs in the Netherlands, the Royal Dutch Society for Physical Therapy developed 20 evidence based guidelines. This includes the guideline 'Intermittent claudication' published in 2003. ${ }^{51}$ Assessing guideline adherence was performed for the guideline 'Lower back pain'. Bekkering et al. showed an adherence to the low back pain guideline for Dutch PTs in only $30 \%$ of the patients. ${ }^{52}$ When PTs had undergone an active implementation strategy, adherence increased by $12 \%$. For the guideline 'Intermittent claudication' no studies on guideline adherence have been performed. It seems however likely that guideline adherence in general is suboptimal. Therefore the assignment of quality indicators is in our view necessary. For example, in Parkinson's disease, guideline recommendations were transformed into 
quality indicators and incorporated into a questionnaire..$^{53}$ This questionnaire was sent to 41 expert PTs and 286 general PTs. The score of the expert panel (35.1, SD 4.2) was significantly higher compared to those of the general PTs (22.2, SD 7.7). The results emphasise that quality improving interventions are needed as guideline adherence is suboptimal.

In the ideal situation, all patients with IC should receive an evidence based standardised form of SET by an exercise specialist. Due to lack of capacity, hospital-based exercise therapy should be reserved for cases with severe (cardiac) comorbidity. For this reason the ClaudicatioNet concept was launched in the Netherlands, in January 2011. ClaudicatioNet is a concept of an integrated care network between PTs, vascular surgeons, and general practitioners. ClaudicatioNet aims to implement nationwide coverage of regional networks for supervised exercise therapy (SET) and lifestyle interventions by PTs. Participating PTs are required to comply with a progressive set of quality standards over a period of 3-4 years. These efforts should result in a transparent, high quality care network as evaluated by objective quality indicators. For example, data on maximal walking ability, quality of life, comorbidities, lifestyle parameters, and cardiovascular risk factors will be recorded in a national database. PTs also need to obtain a set number of continuous medical education (CME) points on topics linked to PAOD.

In our opinion, regular medication compliance should be performed four times a year as part of this standard SET program. If deviations of prescribed medication are noted by the exercise specialist the patient's general practitioner and/or medical specialist should be notified. We hypothesized that due to the frequent (2-3 times a week) contacts of patients with their PTs, as part of a SET program, it is more likely that patients will truly report non-adherence. However, this is not yet substantiated.

Current and future research on SET focuses on the influence of specific comorbidities on prognosis and effect of SET outcome, besides the additional value of SET combined with a percutaneous vascular intervention (PVI).

Currently, in the Netherlands, two multicenter RCT's are including patients to evaluate the effectiveness and cost-effectiveness of SET. The ERASE-trial compares SET with a PVI complementary to SET. ${ }^{54}$ The optimal treatment strategy for IC due to an iliac artery obstruction will be determined in the SUPER-trial..$^{55}$ The SUPER-trial compares SET and deferred PVI in case of SET failure to an immediate PVI. Results have to be awaited and interpreted in view of the limited experience in SET among the participating PTs. In the United States, another large multicenter RCT is being conducted. In approximately 
25 centers 252 patients will be followed over a period of 18 months. ${ }^{56}$ The treatment arms are: 1 . optimal medical care (OMC); 2 . OMC and primary stent placement; 3. OMC and SET; and 4.combined stenting with SET and OMC. In this so called CLEVER trial, the researchers aim to find the optimal treatment strategy for IC for the endpoints maximal walking duration and health-related QoL. In these trials no usage of the future possibilities aimed for in ClaudicatioNet will be measured (i.e. lifestyle coaching, smoking cessation strategies, concurrent use of concomitant comorbidity guidelines like COPD etc.). However, currently it is the best SET we have and results are eagerly waited for.

Although there is little evidence yet, SET could also be effective for patients with critical limb ischemia as an adjuvant to a revascularisation procedure. Kruidenier et al. performed a RCT to investigate the effects of SET after a PVI for patients with all stages of PAOD, mainly IC. ${ }^{57}$ They found that SET following PVI was more effective in increasing maximum walking distance than PVI alone. Badger et al. evaluated the efficacy of an exercise program after arterial bypass surgery for short distance intermittent claudication or critical ischemia. ${ }^{58} \mathrm{SET}$ resulted for this group in an increased maximum walking distance of $175 \%$ compared to $4 \%$ for the group with usual care. These studies indicate that SET is a useful adjunct after a PVI or lower limb bypass surgery. Further research should confirm the clinical effectiveness of SET after a vascular intervention for the subgroup of patients with critical limb ischemia.

\section{CONCLUSION}

Multicomponent treatment of IC should address two important targets; reduction of cardiovascular events (and related morbidity and mortality) and improvement of the poor health-related QoL and functional capacity. Based on current evidence, SET appears to be the primary and most effective intervention for patients with IC. With the implementation of ClaudicatioNet in The Netherlands, a nationwide integrated care network of specialised PTs, enables the delivery of a multicomponent therapy to all patients with IC. Current and future research focuses on the influence of specific comorbidities on prognosis and effect of SET outcome and the potential beneficial effects of SET combined with (minimally) invasive vascular interventions. 


\section{REFERENCES}

1. Hirsch AT, Haskal ZJ, Hertzer NR, et al. ACC/AHA 2005 Practice Guidelines for the management of patients with peripheral arterial disease (lower extremity, renal, mesenteric, and abdominal aortic): a collaborative report from the American Association for Vascular Surgery/ Society for Vascular Surgery, Society for Cardiovascular Angiography and Interventions, Society for Vascular Medicine and Biology, Society of Interventional Radiology, and the ACC/AHA Task Force on Practice Guidelines (Writing Committee to Develop Guidelines for the Management of Patients With Peripheral Arterial Disease): endorsed by the American Association of Cardiovascular and Pulmonary Rehabilitation; National Heart, Lung, and Blood Institute; Society for Vascular Nursing; TransAtlantic Inter-Society Consensus; and Vascular Disease Foundation. Circulation 2006;113:e463-e654

2. Norgren L, Hiatt WR, Dormandy JA, Nehler MR, Harris KA, Fowkes FG. Inter-Society Consensus for the Management of Peripheral Arterial Disease (TASC II). J Vasc Surg 2007;45(Suppl.S):S5-67

3. Fowkes FG, Housley E, Cawood EH, Macintyre CC, Ruckley CV, Prescott RJ. Edinburgh Artery Study: prevalence of asymptomatic and symptomatic peripheral arterial disease in the general population. Int J Epidemiol $1991 ; 20: 384-92$

4. Murabito JM, Evans JC, Nieto K, Larson MG, Levy D, Wilson PW. Prevalence and clinical correlates of peripheral arterial disease in the Framingham Offspring Study. Am Heart J 2002;143:961-5

5. Hooij JD, Stoffers HE, Kester AD, Rinskens $P E$, Kaiser V. Risk factors and cardiovascular diseases associated with asymptomatic peripheral arterial occlusive disease. The Limburg PAOD Study. Scand J Prim Health Care 1998;16:177-82

6. Meijer WT, Hoes AW, Rutgers D, Bots ML, Hofman A, Grobbee DE. Peripheral arterial disease in the elderly: The Rotterdam Study. Arterioscler Thromb Vasc Biol 1998;18:185-92

7. Fauci AS. Harrison's Principles of Internal Medicine. 2nd edn. New York: McGraw-Hill, 1998

8. Kannel WB, McGee DL. Update on some epidemiologic features of intermittent claudication: the Framingham Study. I Am Geriatr Soc 1985;33:13-8
9. Leng GC, Lee $A J$, Fowkes FG, et al. Incidence, natural history and cardiovascular events in symptomatic and asymptomatic peripheral arterial disease in the general population. Int $J$ Epidemiol 1996;25:1172-81

10. Criqui MH. Systemic atherosclerosis risk and the mandate for intervention in atherosclerotic peripheral arterial disease. Am J Cardiol $2001 ; 88: 43 J-7 J$

11. Criqui $M H$, Langer RD, Fronek $A$, et al. Mortality over a period of 10 years in patients with peripheral arterial disease. $N$ Engl J Med 1992;326:381-6

12. Regensteiner JG, Hiatt WR, Coll JR, et al. The impact of peripheral arterial disease on healthrelated quality of life in the Peripheral Arterial Disease Awareness, Risk, and Treatment: New Resources for Survival (PARTNERS) Program. Vasc Med 2008;13:15-24

13. Dumville JC, Lee AJ, Smith FB, Fowkes FG. The health-related quality of life of people with peripheral arterial disease in the community: the Edinburgh Artery Study. Br J Gen Pract 2004;54:826-31

14. Dormandy J, Heeck L, Vig S. Predictors of early disease in the lower limbs. Semin Vasc Surg 1999;12:109-17

15. Chobanian AV, Bakris GL, Black HR, et al. Seventh report of the Joint National Committee on Prevention, Detection, Evaluation, and Treatment of High Blood Pressure. Hypertension 2003;42:1206-52

16. Bonny A, Lacombe $F$, Yitemben $M$, et al. The $2007 \mathrm{ESH} / \mathrm{ESC}$ guidelines for the management of arterial hypertension. I Hypertens 2008;26:825-6

17. Paravastu SC, Mendonca DA, da Silva A. Beta blockers for peripheral arterial disease. Eur J Vasc Endovasc Surg 2009;38:66-70

18. Ray KK, Seshasai SR, Wijesuriya S, et al. Effect of intensive control of glucose on cardiovascular outcomes and death in patients with diabetes mellitus: a meta-analysis of randomized controlled trials. Lancet 2009;373:1765-72

19. Gerstein HC, Miller ME, Genuth S, et al. Longterm effects of intensive glucose lowering on cardiovascular outcomes. N Engl J Med 2011;364:818-28 
20. Heart Protection Study Collaborative Group. Randomized trial of the effects of cholesterollowering with simvastatin on peripheral vascular and other major vascular outcomes in 20,536 people with peripheral arterial disease and other high-risk conditions. J Vasc Surg 2007;45:645-54

21. Armitage JM, Bowman L, Clarke RJ, et al. Effects of homocysteine-lowering with folic acid plus vitamin $\mathrm{B} 12$ vs placebo on mortality and major morbidity in myocardial infarction survivors: a randomized trial. JAMA 2010;303:2486-94

22. Antithrombotic Trialists Collaboration. Collaborative metaanalysis of randomised trials of antiplatelet therapy for prevention of death, myocardial infarction, and stroke in high risk patients. BMJ 2002;324:71-86

23. Newby LK, LaPointe NM, Chen AY, et al. Longterm adherence to evidence-based secondary prevention therapies in coronary artery disease. Circulation 2006;1 13:203-12

24. Watson L, Ellis B, Leng GC. Exercise for intermittent claudication. Cochrane Database Syst Rev 2008;(4):CD000990

25. Kannel WB, Shurtleff $D$. The Framingham Study. Cigarettes and the development of intermittent claudication. Geriatrics 1973;28:61-8

26. Powell JT, Edwards RJ, Worrell PC, Franks PJ, Greenhalgh RM, Poulter NR. Risk factors associated with the development of peripheral arterial disease in smokers: a case-control study. Atherosclerosis 1997;129:41-8

27. Selvin E, Hirsch AT. Contemporary risk factor control and walking dysfunction in individuals with peripheral arterial disease: NHANES 1999-2004. Atherosclerosis 2008;201:42533

28. Jonason T, Bergstrom R. Cessation of smoking in patients with intermittent claudication. Effects on the risk of peripheral vascular complications, myocardial infarction and mortality. Acta Med Scand 1987;221:253-60

29. WHO Press World Health Organization. Global Recommendations on Physical Activity for Health. Geneva: World Health Organization, 2010

30. Thompson PD, Zimet R, Forbes WP, Zhang P. Meta-analysis of results from eight randomized, placebo-controlled trials on the effect of cilostazol on patients with intermittent claudication. Am J Cardiol 2002;90:1314-9
31. De BT, Vander SR, Lehert P, Van BL. Naftidrofuryl for intermittent claudication: meta-analysis based on individual patient data. BMJ 2009;338:b603

32. Erb W. About intermittent walking and nerve disturbances due to vascular disease. Deutsch Z Nervenheilk 1898;13:1-76

33. Larsen OA, Lassen NA. Effect of daily muscular exercise in patients with intermittent claudication. Lancet 1966;2:1093-6

34. Shephard RJ, Balady GJ. Exercise as cardiovascular therapy. Circulation 1999;99:963-72

35. Bartelink ML, Stoffers HE, Biesheuvel CJ, Hoes AW. Walking exercise in patients with intermittent claudication. Experience in routine clinical practice. Br J Gen Pract 2004;54:196200

36. Bendermacher BL, Willigendael EM, Teijink JA, Prins $M H$. Supervised exercise therapy versus non-supervised exercise therapy for intermittent claudication. Cochrane Database Syst Rev 2006;(2):CD005263

37. Spronk S, Bosch JL, den Hoed PT, Veen HF, Pattynama PM, Hunink MG. Intermittent claudication: clinical effectiveness of endovascular revascularization versus supervised hospital-based exercise training - randomized controlled trial. Radiology 2009;250:586-95

38. Creasy TS, McMillan PJ, Fletcher EW, Collin $\mathrm{J}$, Morris PJ. Is percutaneous transluminal angioplasty better than exercise for claudication? Preliminary results from a prospective randomized trial. Eur J Vasc Surg 1990;4:135-40

39. Lundgren F, Dahllof AG, Lundholm K, Schersten $\mathrm{T}$, Volkmann R. Intermittent claudication surgical reconstruction or physical training? A prospective randomized trial of treatment efficiency. Ann Surg 1989;209:346-55

40. Mazari FA, Gulati S, Rahman MN, et al. Early outcomes from a randomized, controlled trial of supervised exercise, angioplasty, and combined therapy in intermittent claudication. Ann Vasc Surg 2010;24:69-79 
41. Greenhalgh RM, Belch JJ, Brown LC, et al. The adjuvant benefit of angioplasty in patients with mild to moderate intermittent claudication (MIMIC) managed by supervised exercise, smoking cessation advice and best medical therapy: results from two randomised trials for stenotic femoropopliteal and aortoiliac arterial disease. Eur J Vasc Endovasc Surg 2008;36:680-8

42. Gardner AW, Katzel LI, Sorkin JD, Goldberg AP. Effects of long-term exercise rehabilitation on claudication distances in patients with peripheral arterial disease: a randomized controlled trial. J Cardiopulm Rehabil 2002;22:192-8

43. Ratliff DA, Puttick $M$, Libertiny G, Hicks RC, Earby LE, Richards T. Supervised exercise training for intermittent claudication: lasting benefit at three years. Eur J Vasc Endovasc Surg 2007;34:322-6

44. Willigendael $E M$, Bendermacher $B L$, van der Berg C, et al. The development and implementation of a regional network of physiotherapists for exercise therapy in patients with peripheral arterial disease, a preliminary report. BMC Health Serv Res 2005;5:49

45. Bendermacher BL, Willigendael EM, Nicolai $\mathrm{SP}$, et al. Supervised exercise therapy for intermittent claudication in a community-based setting is as effective as clinic-based. J Vasc Surg 2007;45:1192-6

46. Nicolai SP, Teijink JA, Prins MH. Multicenter randomized clinical trial of supervised exercise therapy with or without feedback versus walking advice for intermittent claudication. J Vasc Surg 2010;52:348-55

47. van Asselt $A D$, Nicolai SP, Joore MA, Prins $M H$, Teijink JA.Cost-effectiveness of exercise therapy in patients with intermittent claudication: supervised exercise therapy versus a 'go home and walk' advice. Eur J Vasc Endovasc Surg 2011;41:97-103

48. Vahl AC, Reekers JA. The guideline 'Diagnosis and treatment of peripheral artery disease of the lower extremities' of The Netherlands Surgical Society. Ned Tijdschr Geneeskd 2005; 149:1670-4

49. Bartelink ML, Stoffers HEJH, Boutens EJ, Hooii JD, Kaiser V, Boomsma U. Guideline peripheral arterial disease from the Dutch General Practioners Society. Huisarts Wet 2003;46:848-58
50. Hendriks HJM, Bekkering GE, Van Ettekoven $H$, Brandsma JW, van der Wees PJ, De Bie RA. Development and implementation of national practice guidelines: a prospect for continuous quality improvement in physiotherapy: introduction to the method of guideline development. Physiotherapy 2000;86:535-47

51. Bekkering GE. Physiotherapy Guidelines for Low Back Pain. Development, Implementation and Evaluation. Academic thesis. Amsterdam: Vrije Universiteit, 2004

52. Nijkrake MJ, Keus SH, Ewalds H, et al. Quality indicators for physiotherapy in Parkinson's disease. Eur J Phys Rehabil Med 2009;45:23945

53. Nederlands Trial Register. Amsterdam: Academic Medical Center (The Netherlands). 2004 Oct 26. Spronk S. Cost-effectiveness of treatment strategies for intermittent claudication: a multicenter randomized controlled trial. Identifier NTR2249. 2010. See http:// www. trialregister.nl (last checked 28 October 2011)

54. Nederlands Trial Register. Amsterdam: Academic Medical Center (The Netherlands). 2004 Oct 26. Koelemay MJW. SUPERvised exercise therapy or immediate PTA for intermittent claudication in patients with an iliac artery obstruction: a randomized controlled trial. SUPER study. Identifier NTR2776. 2011. See http://www.trialregister.nl (last checked 28 October 2011)

55. Murphy TP, Hirsch AT, Ricotta JJ, et al. The claudication: exercise vs. endoluminal revascularization (CLEVER) study: rationale and methods. J Vasc Surg 2008;47:1356-63

56. Kruidenier LM, Nicolai SP, Rouwet EV, Peters RJ, Prins MH, Teijink JA. Additional supervised exercise therapy after a percutaneous intervention for peripheral arterial disease: a randomised clinical trial. J Vasc Interv Radiol $2011 ; 22: 961-8$

57. Badger SA, Soong CV, O'Donnell ME, Boreham CA, McGuigan KE. Benefits of a supervised exercise program after lower limb bypass surgery. Vasc Endovasc Surg 2007;41:27-32 



\section{CHAPTER 3}

\section{WHEN IS SUPERVISED EXERCISE \\ THERAPY INDICATED FOR \\ INTERMITTENT CLAUDICATION?}

A NATIONWIDE SURVEY
AMONG VASCULAR SURGEONS

G.J. Lauret

H.C. van Dalen

H.J. Hendriks

S.M. van Sterkenburg

M.J. Koelemay

C.J. Zeebregts

R.J. Peters

J.A. Teijink

Eur J Vasc Endovasc Surg 2012;43(3):308-12 


\section{ABSTRACT}

\section{Objectives}

Although international guidelines state that supervised exercise therapy (SET) should be offered to all patients with intermittent claudication (IC), SET appears to be underutilised in clinical practice. The aim of this study was to document current opinions of Dutch vascular surgeons on SET as treatment option for peripheral arterial occlusive disease (PAOD).

\section{Materials and methods}

Vascular surgeons and fellows in vascular surgery were asked to complete a 24-question survey either at the 2011 Annual Meeting of the Dutch Society for Vascular Surgery or online.

\section{Results}

Ninety-one participants, including 83 vascular surgeons (51\% of all Dutch vascular surgeons), completed the survey. The respondents would refer $75.4 \%$ of newly diagnosed patients with IC for SET. SET was considered less useful in patients with IC and major (cardiopulmonary) comorbidity or a significant iliac artery stenosis. In critical limb ischemia, the combination of SET and angioplasty was considered useful in $71.9 \%$. Respondents regarded patient satisfaction (63.3\%) and improvement in painfree or maximal walking ability $(26.6 \%)$ as clinically most relevant goals of SET. Most $(84.4 \%)$ agreed that SET should also include lifestyle management.

\section{Conclusion}

Although the vast majority of Dutch vascular surgeons consider SET as an important treatment option for PAOD, SET should receive more emphasis in clinical practice since arguments not to refer for SET are outdated. Furthermore, vascular surgeons agree that lifestyle management should be integrated in SET. 


\section{INTRODUCTION}

In the management of intermittent claudication (IC), supervised exercise therapy (SET) leads to superior results (walking distances, quality of life) compared to a single walking advice. ${ }^{1,2}$ When compared to angioplasty, patients with IC benefited equally from either angioplasty or hospital-based SET after a 12-month follow-up., ${ }^{3,4}$ SET is safe and does not cause morbidity or mortality. For this reason, initial management of IC should consist of cardiovascular risk management and SET, as clearly stated in contemporary international guidelines.

Community-based SET seems as efficacious as hospital-based SET, is cost-effective and should therefore be available for all patients with IC., Nonetheless, in clinical practice, SET seems underutilised because of limited availability of physical or exercise therapists with specific knowledge on peripheral arterial occlusive disease (PAOD) and/or a SET treatment protocol. For this reason Nicolaï et al. extensively trained all participating community-based physical therapists before they were allowed to participate in the multicenter randomised controlled ExitPAD trial. ${ }^{2}$ Another explanation for the underutilisation of SET could be the differing views on the indications for SET, such as concomitant significant cardiopulmonary comorbidity, patient's age, aortic or iliac artery obstruction as cause of IC, or SET in combination with revascularisation for critical limb ischemia (CLI). Furthermore, in severe or invalidating cases of IC vascular surgeons often perform a vascular intervention. However, a solid definition of 'severely disabling' or 'invalidating' IC is lacking.

The aim of this study is to document current opinions of vascular surgeons and fellows in vascular surgery on SET as treatment option for PAOD. We specifically wanted to find out to what extent and on which criteria Dutch vascular surgeons decide to offer or deny SET to patients with PAOD.

\section{MATERIALS AND METHODS}

In spring 2011, Dutch vascular surgeons and fellows in vascular surgery were asked to complete a 25-question survey regarding SET as treatment option for PAOD. Participants could complete the survey on the Annual Meeting of the Dutch Society for Vascular Surgery or on a secure online website. Most questions (21/25) were defined as statements that participants could agree or disagree with. One question was a multiple answer question, three were open-ended questions (regarding the number of SET referrals per hospital, percentage of all patients with IC referred and criteria for successful conservative therapy). 


\section{Data extraction}

Data extraction was performed by two independent investigators (GL, HvD). All surveys were analysed for missing answers and the number of responses per question were noted. If answers were unclear, the same investigators determined if the answer could be classified to a specific category. If this was not possible or in case of disagreement between the two investigators the answer was excluded from analysis. For the openended question 'when is conservative therapy considered successful', answers were classified by each of the two investigators. In case of disagreement, concurrence was sought by means of discussion. For the remaining open-ended questions the numbers and percentages were extracted. When a range of numbers or percentages was given, the mean was calculated and used as definitive answer.

\section{Statistical Analysis}

Data were analysed for both descriptive and analytical statistics. Because of a skewed distribution of continuous variables (referral data), evaluated by a onesample Kolmogorov-Smirnov test, we performed a Mann-Whitney U Test to determine statistical difference between two groups. Medians and interquartile ranges (IQR) were calculated to describe the spread of data. A p-value $<0.05$ was considered to indicate statistical significance. All analyses were performed using IBM SPSS Statistics Version 19 (SPSS Inc., Chicago).

\section{RESULTS}

\section{Participants}

In total 91 participants, including 83 vascular surgeons, completed the survey. This comprises $51 \%$ of all Dutch vascular surgeons and seems therefore representative as nationwide survey. All respondents are further classified by gender, age group, professional career and hospital setting (Table 1).

\section{Referral information}

The number of new patients with IC, treated annually, was estimated to be 476 per hospital (median 400, IQR 260-640). No significant ( $p=0.626$ ) difference was found between academic and non-academic hospitals. Overall, 88 respondents estimated to refer on average $75.4 \%$ (median $80 \%$, IQR 66.3 - 95.0) of their patients with IC for SET. 
TABLE 1 Baseline characteristics

\begin{tabular}{|c|c|}
\hline & Number of Responses (\%) \\
\hline Total respondents & $91(100)$ \\
\hline \multicolumn{2}{|l|}{ Gender } \\
\hline Men & $78(85.7)$ \\
\hline Women & $13(14.3)$ \\
\hline \multicolumn{2}{|l|}{ Age } \\
\hline $30-40$ & $23(25.3)$ \\
\hline $40-50$ & $40(44.0)$ \\
\hline $50-60$ & $20(20.9)$ \\
\hline $60+$ & $9(9.9)$ \\
\hline \multicolumn{2}{|l|}{ Professional career } \\
\hline Fellow in vascular surgery & $8(8.8)$ \\
\hline Vascular surgeon & $83(91.2)$ \\
\hline Professor in vascular surgery & $3(3.3)$ \\
\hline \multicolumn{2}{|l|}{ Hospital setting } \\
\hline Academic hospital & $15(16.5)$ \\
\hline Non-academic hospital & $76(83.5)$ \\
\hline
\end{tabular}

\section{SET indications}

Table 2 lists the attitude towards indications for SET. Most respondents had a positive attitude towards SET in general. However, continuation of SET after three months of treatment without functional improvement, was only preferred by $60.0 \%$ of the respondents.

Most respondents did not consider a maximum walking distance of $<100 \mathrm{~m}$ or age older than 80 years a contraindication for SET. However, in patients with a significant iliac artery stenosis $(70.8 \%)$, patients with a coinciding chronic pulmonary condition (e.g. COPD) (65.9\%) or chronic heart failure (69.8\%), SET was less frequently thought to be useful.

As additional treatment before or after angioplasty for CLI, SET was thought to be useful by $71.9 \%$ of the respondents while $64.8 \%$ would recommend SET as an adjunct to bypass surgery. 
TABLE 2 Level of agreement to statements regarding the use of SET

\section{Agreement}

$\%$

SET in general

SET is more effective than a single walking advice 100

In addition to CVRM, SET is the primary treatment for IC

Community-based SET is as effective as hospital-based SET

Feedback of the physical therapist contributes to patient management

Continuation of SET is useful if patients do not improve in the

60.0

first 3 months of treatment

SET for intermittent claudication is useful in:

- patients with a maximal walking distance of $<100 \mathrm{~m}$

- patients older than 80 years

- patients who underwent angioplasty

- intermittent claudication caused by a significant iliac stenosis

- patients with not-decompensated chronic heart failure

- patients with a chronic pulmonary condition (e.g. COPD)

SET for critical limb ischemia is useful in addition to:

- angioplasty

- peripheral bypass surgery

64.8

$(57 / 86)$

SET: supervised exercise therapy; IC: intermittent claudication; CVRM: cardiovascular risk management

\section{Definition of successful conservative management}

In total, 91 respondents gave 109 categorised answers to the open question; when is conservative management (cardiovascular risk management and SET) for IC considered successful? Patient satisfaction (63.3\% of all answers) and progress in pain-free or maximal walking ability ( $26.6 \%$ of all answers) were the most prevalent criteria to determine successful conservative management (Table 3).

\section{Invalidating IC}

The decision to perform angioplasty for IC is determined by the interpretation of 'severely disabling' or 'invalidating' IC. For this reason, respondents were asked which variables contributed to the decision to perform angioplasty with a multiple-answer question. Limitations in daily life (LDL), patient reported walking ability (WA-P) and walking ability determined in the vascular laboratory (WA-V) were possible answer options. Respondents could also mention other factors influencing their decision to perform angioplasty. The combination of LDL, WA-P and WA-V was thought to 
contribute the most to choosing an angioplasty (32.2\%) followed by LDL alone (23.3\%), LDL in combination with WA-P $(20.0 \%)$ and $L D L$ in combination with WA-V $(12.2 \%)$ (Table 4). Other mentioned responses were anatomic location of the stenosis/occlusion $(n=3)$, type of stenosis $(n=2)$, effect of three months of SET $(n=1)$, cardiopulmonary condition ( $n=1)$ and patency of the procedure $(n=1)$. LDL alone or in combination with WA-P or WA- $V$ was mentioned in $87.8 \%$ of all answers $(n=79 / 90)$. Walking ability (WA-P or WA-V) was mentioned in $76.7 \%$ of all answers $(n=69 / 90)$. Therefore the patient's LDL seems to be more contributing to the decision to perform an angioplasty then the patient's walking ability.

TABLE 3 When is conservative therapy considered successful in IC?

\begin{tabular}{|lcc|}
\hline Patient satisfaction & $\%$ & $\mathrm{n}$ \\
\hline Progress in pain-free or maximal walking ability & 63.3 & $(69 / 109)$ \\
\hline Progress in quality of life & 26.6 & $(29 / 109)$ \\
\hline Increased ankle-brachial index & 2.8 & $(3 / 109)$ \\
\hline No further decline in pain-free or maximal walking ability & 2.8 & $(3 / 109)$ \\
\hline Adjustment of patient's lifestyle & 1.8 & $(2 / 109)$ \\
\hline ADL recovery & 1.8 & $(2 / 109)$ \\
\hline
\end{tabular}

IC: intermittent claudication; ADL: activities of daily living

TABLE 4 Determinants for angioplasty in invalidating IC

\begin{tabular}{|lcc|}
\hline LDL + WA-P + WA-V & $\%$ & $n$ \\
\hline LDL & 32.2 & $(29 / 90)$ \\
\hline$L L+W A-P$ & 23.3 & $(21 / 90)$ \\
\hline LD + WA-V & 20.0 & $(18 / 90)$ \\
\hline WA-P + WA-V & 12.2 & $(11 / 90)$ \\
\hline WA-P & 5.6 & $(5 / 90)$ \\
WA-V & 5.6 & $(5 / 90)$ \\
\hline
\end{tabular}

IC: intermittent claudication; LDL: Limitations in Daily Life; WA-P: Patient reported Walking Ability; WA-V: Vascular Laboratory determined Walking Ability

\section{Content of SET}

Most participants agreed that supervised walking exercise (100\%) and lifestyle interventions (84.4\%) should be part of supervised exercise rehabilitation. Assessment 
of medication adherence, side effects and blood glucose levels was not thought to be contributing to supervised exercise rehabilitation (Table 5)

TABLE 5 Supervised exercise rehabilitation should contain...

\begin{tabular}{lcc} 
& \multicolumn{2}{c}{ Agreement } \\
\cline { 2 - 3 } & $\%$ & $\mathrm{n}$ \\
\hline Supervised walking exercise & 100 & $(90 / 90)$ \\
Lifestyle interventions (e.g. smoking cessation, dietary advice) & 84.4 & $(76 / 90)$ \\
Supervised alternative exercise (e.g. cycling, strength training) & 62.2 & $(56 / 90)$ \\
Cardiopulmonary risk assessment & 54.4 & $(49 / 90)$ \\
Assessment of used medication & 45.6 & $(41 / 90)$ \\
Assessment of medication adherence & 32.2 & $(29 / 90)$ \\
Assessment of drug side effects & 22.2 & $(20 / 90)$ \\
Assessment of blood glucose levels (in case of DM type 2) & 18.9 & $(17 / 90)$
\end{tabular}

DM: diabetes mellitus

\section{DISCUSSION}

The TASC II Inter-Society Consensus for the Management of Peripheral Arterial Disease recommends SET as part of the initial treatment for all patients with PAOD. ${ }^{5}$ As shown in our study, the vast majority of Dutch vascular surgeons and fellows in vascular surgery have a positive attitude towards SET as treatment option for IC and even CLI. More than three-quarters of new patients with IC are estimated to be referred for SET. Results could be influenced by responder bias, most likely in favour of a positive attitude towards SET. Therefore, the found attitude towards SET may in fact be less positive in real life. In a study on the use of SET in the United Kingdom, a large spread in proportion of patients referred for SET was found. ${ }^{9}$ Furthermore, only $24 \%$ of resident surgeons had access to supervised exercise in the UK. In the Netherlands, communitybased physical therapists are readily available and can provide SET according the guidelines of the Royal Dutch Society for Physical Therapy, although not all physical therapists are adequately schooled.

Besides limited availability of qualified therapists providing SET, the infrastructure of the health care system can also be a reason for underutilisation of SET. If patients need to pay a significant amount of SET treatments one might argue that angioplasty would be more attractive from a patient point-of-view. In the Netherlands, the first 12 treatments in 2011 (20 treatments in 2012) are covered by an additional health care insurance. 
The basic health care insurance covers all additional SET treatments for IC until one year of training. Since most patients have an additional health care insurance, SET does not lead to any additional costs for the patient in the Netherlands. However, in other countries this could surely be one of the reasons of SET underutilization.

This study showed that the criteria not to refer for SET are largely based on existence of major comorbidity (e.g. pulmonary, cardiac) or a significant iliac stenosis causing IC. Apparently, the respondents did not consider SET to be successful in these particular patients. This could be explained by concern of major complications or the belief that SET is ineffective to improve walking ability or quality of life in these particular patients. Nonetheless, COPD has shown to be present in $16 \%$ of all patients with PAOD for which pulmonary rehabilitation (PR) is strongly indicated to increase physical functioning, reduce hospital admissions, reduce respiratory symptoms and improve ADL-activities. ${ }^{10,12}$ PR consists primarily of supervised aerobic exercise (e.g. treadmill walking, cycling) in a moderate to severe intensity. Although treatment regimens of PR are not identical to SET for IC, a significantly improved health-related quality of life and walking distances were found for PR after 12 months of exercise. ${ }^{13}$

Coronary artery disease has shown to be present in $46-58 \%$ of all patients with PAOD, of which $22 \%$ even had active ischemia. ${ }^{10,14}$ For these coronary conditions, requiring hospitalisation or cardiac revascularisation procedures, cardiac rehabilitation in the form of a moderate intensity aerobic exercise training was associated with a 21$34 \%$ decrease in 5-year mortality. ${ }^{15}$ Likewise for congestive heart failure, cardiac rehabilitation is also advised with a class $\mathrm{lb}$ recommendation. ${ }^{16}$ For these patients exercise training resulted in a modest $(11 \%)$ reduction in the adjusted risk for all-cause mortality. ${ }^{17}$

Exercise training also appears to be safe in patients with coronary heart disease. In a systematic review, more than 2 million exercise hours, performed by 51.000 patients with coronary heart disease, were analysed. ${ }^{18}$ In total, 21 cardiac arrests (of which 3 fatal) and 8 nonfatal myocardial infarctions (MI) were found, equivalent to 1 cardiac arrest per 112.000 exercise hours, 1 acute MI per 300.000 exercise hours and 1 fatality per 800.000 exercise hours. It is questionable whether these rates are higher than the naturally occurring rates of these events. It is clear that SET should be offered to patients with coexistence of IC and congestive HF, chronic pulmonary conditions or coronary conditions, although patient monitoring and exercise intensity should be adjusted to the patient's conditions. 
In case of a significant iliac artery stenosis causing IC, studies comparing SET to angioplasty are lacking. Currently, in the Netherlands, the multicenter randomised controlled SUPER trial is enrolling 300 patients to investigate which treatment modality should be performed: SET and deferred angioplasty (in case of SET failure) or immediate angioplasty. ${ }^{19}$ The complementary effects of SET and angioplasty have been described previously. In the MIMIC trial, patients with femoropopliteal and aorto-iliac arterial disease were randomised to receive either PTA or no PTA against a background of SET and best medical therapy. ${ }^{20}$ The maximum walking distance after 24 months of follow up was $38 \%$ greater in the PTA group for the femoropopliteal trial $(95 \% ; \mathrm{Cl} 1-90)(\mathrm{p}=0.04)$ and $78 \%$ greater in the PTA group for the aorto-iliac trial $(95 \%$; $\mathrm{Cl} 0-216)(\mathrm{p}=0.05)$. No benefits were found regarding health-related quality of life. Although more studies are needed to determine the effect of SET for this particular group of claudicants, SET seems to show beneficial effects when combined with a vascular intervention.

Although there is little evidence yet, SET may also be effective as adjuvant to a revascularisation procedure in patients with invalidating IC or CLI. Kruidenier et al. performed a RCT to investigate the effects of SET after angioplasty for patients with all stages of PAOD. ${ }^{21}$ They found that SET following angioplasty was more effective in increasing maximum walking distance than angioplasty alone. Badger et al. evaluated the efficacy of an exercise program after arterial bypass surgery for short distance intermittent claudication or $\mathrm{CLI} .{ }^{22} \mathrm{SET}$ resulted for this group in an increased maximum walking distance of $175 \%$ compared to $4 \%$ for the group with usual care. Although not all included patients had CLI, these studies indicate that SET could be a useful adjunct after angioplasty or bypass surgery. In this study, SET was considered by $71.9 \%$ of the respondents in addition to angioplasty and $64.8 \%$ in addition to bypass surgery. Although more evidence is needed, SET seems to have a role in the treatment of CLI.

One might argue that besides smoking cessation, regaining physical activity in patients with PAOD, is the most important determinant to reduce major cardiovascular events and mortality. Healthy individuals engaging in moderate-intensity physical activity (150 $\mathrm{min} / \mathrm{wk}$ ) had a $14 \%$ lower coronary heart disease risk compared with $20 \%$ reduction in those engaging $300 \mathrm{~min} / \mathrm{wk}$ of exercise. ${ }^{23}$ Therefore, any physical activity is probably better than none and additional benefit occurs with more physical activity. The respondents agreed that lifestyle interventions, such as a minimal intervention strategy for smoking cessation, should be integrated in SET to further reduce progression of atherosclerosis, cardiovascular events and even mortality. Given the frequent contacts of physical or exercise therapist, a future role as lifestyle coaches for these specialists is conceivable. 
Currently, in the Netherlands, a nationwide network (ClaudicatioNet) of vascular surgeons and physical therapists is being implemented. National coverage should exist in 2015. In this concept, physical therapists are trained in providing SET and lifestyle interventions tailored to the patient's needs. To remain network participation they need to comply with quality standards such as reporting patient progress to a national database, complying with audits and continuous medical education. After completion of a regional network, vascular surgeons are expected to refer only to those physical therapists, accredited by ClaudicatioNet. Results are eagerly awaited for, but this initiative will further improve the quality of conservative management of PAOD in the Netherlands.

\section{CONCLUSION}

SET is considered an important component of the management of PAOD. Conditions that are perceived as contraindications for SET, including cardiopulmonary morbidity, are in fact additional indications for participation in an exercise program. Limited availability of qualified therapists and financial barriers in healthcare systems can add to this underuse. Ideally, therapists providing SET would also play a role in lifestyle management of patients with PAOD. 


\section{REFERENCES}

1. Bendermacher BL, Willigendael EM, Teijink JA, Prins $M H$. Supervised exercise therapy versus non-supervised exercise therapy for intermittent claudication. Cochrane Database Syst Rev 2006;2.CD005263.

2. Nicolai SP, Teijink JA, Prins MH. Multicenter randomized clinical trial of supervised exercise therapy with or without feedback versus walking advice for intermittent claudication. J Vasc Surg 2010 Aug;52(2):348-55.

3. Spronk S, Bosch JL, den Hoed PT, Veen HF, Pattynama PM, Hunink MG. Intermittent claudication: clinical effectiveness of endovascular revascularization versus supervised hospital-based exercise trainingrandomized controlled trial. Radiology 2009 Feb;250(2):586-95.

4. Creasy TS, McMillan PJ, Fletcher EW, Collin $\mathrm{J}$, Morris PJ. Is percutaneous transluminal angioplasty better than exercise for claudication? Preliminary results from a prospective randomised trial. Eur J Vasc Surg $1990 \mathrm{Apr} ; 4(2): 135-40$.

5. Norgren L, Hiatt WR, Dormandy JA, Nehler MR, Harris KA, Fowkes FG. Intersociety consensus for the management of peripheral arterial disease (TASC II). J Vasc Surg 2007 Jan;45(Suppl. S):S5-67.

6. Hirsch AT, Haskal ZJ, Hertzer NR, Bakal CW, Creager MA, Halperin JL, et al. ACC/AHA 2005 Practice Guidelines for the management of patients with peripheral arterial disease (lower extremity, renal, mesenteric, and abdominal aortic): a collaborative report from the American Association for Vascular Surgery/ Society for Vascular Surgery, Society for Cardiovascular Angiography and Interventions, Society for Vascular Medicine and Biology, Society of Interventional Radiology, and the ACC/AHA Task Force on Practice Guidelines (Writing Committee to Develop Guidelines for the Management of Patients With Peripheral Arterial Disease): endorsed by the American Association of Cardiovascular and Pulmonary Rehabilitation; National Heart, Lung, and Blood Institute; Society for Vascular Nursing; TransAtlantic Inter-society Consensus; and Vascular Disease Foundation. Circulation 2006 Mar 21;113(11). 463-654.
7. van Asselt $A D$, Nicolai $S P$, Joore $M A$, Prins $M H$ Teijink JA. Cost-effectiveness of exercise therapy in patients with intermittent claudication: supervised exercise therapy versus a 'go home and walk' advice. Eur J Vasc Endovasc Surg 2011 Jan;41(1):97-103.

8. Bendermacher BL, Willigendael EM, Nicolai $S P$, Kruidenier LM, Welten RJ, Hendriks E, et al. Supervised exercise therapy for intermittent claudication in a community-based setting is as effective as clinic-based. J Vasc Surg 2007 Jun;45(6):1192-6.

9. Shalhoub J, Hamish M, Davies AH. Supervised exercise for intermittent claudication e an under-utilised tool. Ann R Coll Surg Engl 2009 Sep;91(6): 473-6.

10. von Kemp K, Van den Brande P, Peterson $T$, Waegeneers S, Scheerlinck T, Danau W, et al. Screening for concomitant diseases in peripheral vascular patients. Results of a systematic approach. Int Angiol 1997 Jun;16(2): 114-22.

11. Casaburi R, ZuWallack R. Pulmonary rehabilitation for management of chronic obstructive pulmonary disease. N Engl J Med 2009 Mar 26;360(13):1329-35.

12. Ries AL, Bauldoff GS, Carlin BW, Casaburi $\mathrm{R}$, Emery CF, Mahler DA, et al. Pulmonary rehabilitation: Joint ACCP/AACVPR Evidencebased Clinical Practice Guidelines. Chest 2007 May;131(5 Suppl.). 4S-42S.

13. Verrill D, Barton C, Beasley W, Lippard WM. The effects of short-term and longterm pulmonary rehabilitation on functional capacity, perceived dyspnea, and quality of life. Chest 2005 Aug; 128(2):673-83.

14. Aronow WS, Ahn C. Prevalence of coexistence of coronary artery disease, peripheral arterial disease, and atherothrombotic brain infarction in men and women > or . 62 years of age. Am J Cardiol 1994 Jul 1;74(1):64-5.

15. Suaya JA, StasonWB, Ades PA, Normand SL, Shepard DS. Cardiac rehabilitation and survival in older coronary patients. J Am Coll Cardiol 2009 Jun 30;54(1):25-33. 
16. Jessup $M$, Abraham WT, Casey DE, Feldman AM, Francis GS, Ganiats TG, et al. 2009 focused update: ACCF/AHA Guidelines for the diagnosis and management of heart failure in adults: a report of the American College of Cardiology Foundation/American Heart Association Task Force on Practice Guidelines: developed in collaboration with the International Society for Heart and Lung Transplantation. Circulation 2009 Apr 14;119(14): 1977-2016.

17. O'Connor CM, Whellan DJ, Lee KL, Keteyian SJ, Cooper LS, Ellis SJ, et al. Efficacy and safety of exercise training in patients with chronic heart failure: HF-ACTION randomized controlled trial. J Am Med Assoc 2009 Apr 8;301(14):1439-50.

18. Van Camp SP, Peterson RA. Cardiovascular complications of outpatient cardiac rehabilitation programs. J Am Med Assoc 1986 Sep 5;256(9):1160-3.

19. SUPERvised exercise therapy or immediate PTA for intermittent claudication in patients with an iliac artery obstruction: a randomized controlled trial. SUPER study. ClinicalTrials.gov identifier: NCT01385774, www.ClincalTrials. gov; 2011.
20. Greenhalgh RM, Belch JJ, Brown LC, Gaines PA, Gao L, Reise JA, et al. The adjuvant benefit of angioplasty in patients with mild to moderate intermittent claudication (MIMIC) managed by supervised exercise, smoking cessation advice and best medical therapy: results from two randomised trials for stenotic femoropopliteal and aortoiliac arterial disease. Eur J Vasc Endovasc Surg 2008 Dec;36(6):680-8.

21. Kruidenier LM, Nicolai SP, Rouwet EV, Peters RJ, Prins MH, Teijink JA. Additional supervised exercise therapy after as percutaneous intervention for peripheral arterial disease: a randomised clinical trial 2011 (accepted) ed.

22. Badger SA, Soong CV, O'Donnell ME, Boreham CA, McGuigan KE. Benefits of a supervised exercise program after lower limb bypass surgery. Vasc Endovascular Surg 2007 Feb;41(1):27-32.

23. Sattelmair J, Pertman J, Ding EL, Kohl III HW, Haskell W, Lee IM. Dose response between physical activity and risk of coronary heart disease: a meta-analysis. Circulation 2011 Aug 16;124(7):78-95. 



\section{CHAPTER 4}

\section{MODES OF EXERCISE TRAINING FOR INTERMITTENT CLAUDICATION}

G.J. Lauret

F. Fakhry

H.J.P. Fokkenrood

M.G.M. Hunink

J.A.W. Teijink

S. Spronk

Cochrane Database of Systematic Reviews, 2014, Issue 7 


\section{ABSTRACT}

\section{Background}

According to international guidelines and literature, all patients with intermittent claudication should receive an initial treatment of cardiovascular risk modification, lifestyle coaching, and supervised exercise therapy. In most studies, supervised exercise therapy consists of treadmill or track walking. However, alternative modes of exercise therapy have been described and yielded similar results to walking. Therefore, the following question remains: Which exercise mode gives the most beneficial results?

\section{Objectives}

Primary objective: To assess the effects of different modes of supervised exercise therapy on the maximum walking distance (MWD) of patients with intermittent claudication. Secondary objectives: To assess the effects of different modes of supervised exercise therapy on pain-free walking distance (PFWD) and health-related quality of life scores (HR-QoL) of patients with intermittent claudication.

\section{Search methods}

The Cochrane Peripheral Vascular Diseases Group Trials Search Coordinator searched the Cochrane Peripheral Vascular Diseases Group Specialised Register (July 2013); CENTRAL(2013, Issue6), inTheCochraneLibrary; andclinical trialsdatabases. Theauthors searched the MEDLINE (1946 to July 2013) and Embase (1973 to July 2013) databases and reviewed the reference lists of identified articles to detect other relevant citations.

\section{Selection criteria}

Randomised controlled trials of studies comparing alternative modes of exercise training or combinations of exercise modes with a control group of supervised walking exercise in patients with clinically determined intermittent claudication. The supervised walking programme needed to be supervised at least twice a week for a consecutive six weeks of training.

\section{Data collection and analysis}

Two authors independently selected studies, extracted data, and assessed the risk of bias for each study. Because of different treadmill test protocols to assess the maximum or pain-free walking distance, we converted all distances or walking times to total metabolic equivalents (METs) using the American College of Sports Medicine (ACSM) walking equation. 


\section{Main results}

In this review, we included a total of five studies comparing supervised walking exercise and alternative modes of exercise. The alternative modes of exercise therapy included cycling, strength training, and upper-arm ergometry. The studies represented a sample size of 135 participants with a low risk of bias. Overall, there was no clear evidence of a difference between supervised walking exercise and alternative modes of exercise in maximum walking distance (8.15 METs, $95 \%$ confidence interval (Cl) -2.63 to 18.94 , $\mathrm{P}=0.14$, equivalent of an increase of 173 metres, $95 \% \mathrm{Cl}-56$ to 401 ) on a treadmill with no incline and an average speed of $3.2 \mathrm{~km} / \mathrm{h}$, which is comparable with walking in daily life.

Similarly, there was no clear evidence of a difference between supervised walking exercise and alternative modes of exercise in painfree walking distance $16.42 \mathrm{MET}$, $95 \% \mathrm{Cl}-1.52$ to $14.36, \mathrm{P}=0.11$, equivalent of an increase of 136 metres, $95 \% \mathrm{Cl}$ -32 to 304$)$.

Sensitivity analysis did not alter the results significantly. Quality of life measures showed significant improvements in both groups; however, because of skewed data and the very small sample size of the studies, we did not perform a meta-analysis for healthrelated quality of life and functional impairment.

\section{Authors' conclusions}

There was no clear evidence of differences between supervised walking exercise and alternative exercise modes in improving the maximum and pain-free walking distance of patients with intermittent claudication. More studies with larger sample sizes are needed to make meaningful comparisons between each alternative exercise mode and the current standard of supervised treadmill walking. The results indicate that alternative exercise modes may be useful when supervised walking exercise is not an option for the patient. 


\section{PLAIN LANGUAGE SUMMARY}

\section{Modes of exercise training for intermittent claudication}

Intermittent claudication is a cramping leg pain that develops during exercise and is relieved by a short period of rest. It is caused by inadequate blood flow to the muscles of the leg because of atherosclerosis (hardening and narrowing of the arteries). Intermittent claudication is closely associated with other vascular diseases, such as a heart attack or stroke. Therefore, all patients with intermittent claudication should receive cardiovascular risk management and lifestyle coaching to reduce cardiovascular risk factors.

To improve the walking capacity and quality of life, supervised exercise therapy is the primary treatment according to the current scientific evidence. Community-based supervised exercise appears to be at least as effective as programmes provided in a hospital setting. In the literature, supervised exercise therapy usually consists of treadmill walking. However, alternative modes of exercise therapy (e.g. cycling, strength training) have been described, with beneficial effects on walking capacity and quality of life. Therefore, the following question remains: Which exercise mode gives the most beneficial results?

The present review shows that there are few studies comparing alternative modes of exercise training to the standard of supervised walking exercise. The review authors identified five studies that randomised a total of 135 participants. The alternative modes of exercise therapy included cycling, strength training, and upper-arm ergometry. Comparing these alternative modes of exercise with supervised walking exercise showed no clear evidence of a difference in maximum or pain-free walking distance between the groups. Quality of life measures showed significant improvements in both groups; however, we could not make a direct comparison because of limited data.

This review shows that alternative modes of exercise therapy seem to yield similar results to supervised walking therapy. Therefore, they may be considered useful when supervised walking exercise is not an option for the patient. However, more randomised controlled trials are needed to make a meaningful comparison between the different modes of exercise therapy. 


\section{BACKGROUND}

\section{Description of the condition}

Peripheral arterial occlusive disease (PAOD) is a chronic arterial occlusive disease caused by progressive atherosclerosis. Several arterial segments can be affected, such as the aorta; iliac; and femoral, popliteal, and tibial arteries in the limbs. The most common symptom is intermittent claudication (IC), defined as a cramping pain in the muscles of the leg(s) that occurs during exercise and is relieved by a short period of rest. Because of this condition, patients have a diminished maximum and pain-free walking capacity, leading to diminished health-related quality of life.'

The incidence of IC increases with age, with an annual incidence rate of $0.7 \%, 3.9 \%$, and $10.6 \%$ among 35 - to 44 -year-old men, 45- to 54-year-old men, and 55- to 64 -year-old men, respectively. ${ }^{2}$ In women, the incidence rates are approximately $50 \%$ lower. ${ }^{2}$ IC restricts patients' activity and mobility and considerably reduces their healthrelated quality of life. ${ }^{1,3}$ In addition, because of the ongoing generalised atherosclerotic process, IC is closely associated with cardiovascular morbidity and mortality. Patients with IC have a five-year all-cause mortality rate of $10 \%$ to $15 \%$ and a $20 \%$ chance of a non-fatal cardiovascular event. ${ }^{4}$ When IC progresses to critical limb ischaemia, an even higher mortality rate of $25 \%$ after one year is found. ${ }^{5}$

\section{Description of the intervention}

Because of the serious health risks, all patients with IC should receive a multicomponent therapy consisting of cardiovascular risk modification, lifestyle coaching, and exercise therapy. ${ }^{5}$ Several randomised controlled trials and systematic reviews compared walking exercise supervised by a physical or exercise therapist to non-supervised exercise, usual care, placebo, single walking advice, endovascular interventions, or bypass surgery. ${ }^{6-10}$

The current evidence supports supervised exercise therapy as the primary treatment for improvement of walking capacity and health-related quality of life in patients with IC. Furthermore, community-based supervised exercise appears to be at least as efficacious as programmes provided in a hospital setting. ${ }^{11-13}$ However, less attention has been paid to the mode of (supervised) exercise. Besides walking, alternative modes of supervised exercise training, such as cycling, upper-extremity cycle ergometer exercise and strength training exist and are associated with a significantly improved walking capacity. ${ }^{14-16}$ 


\section{How the intervention might work}

A number of potential mechanisms have been suggested for the reduced functional capacity in IC, such as blood flow limitation due to arterial obstruction, disruption of endothelial function, altered skeletal muscle phenotype by mitochondrial dysfunction, increased blood viscosity, and inflammatory activation. ${ }^{17}$ Exercise has the potential to reverse these pathological events and thereby interrupt the clinical course toward disability. ${ }^{17}$

\section{Why it is important to do this review}

In most studies, supervised exercise programmes involve treadmill or track walking that is of sufficient intensity to bring on claudication pain. Walking exercise is alternated with rest over the course of a 30- to 60-minute session. Exercise therapy for IC is recommended at least three times a week for three months, although there does not seem to be a clear dose-response relationship between exercise volume or intensity and symptom relief. 5,18 Unfortunately, some groups of patients with IC are not capable of completing the exercise protocol because of concomitant comorbidities, such as arthrosis, chronic obstructive pulmonary disease, stroke, or cardiac complaints. For these patients, an adjusted protocol or alternative exercise regime may be proposed.

Recently, a systematic review comparing any mode of exercise, whether supervised or unsupervised, was published. ${ }^{18}$ It suggested that alternative modes of aerobic exercise, other than walking, appear equally beneficial compared to walking exercise, while the effects of progressive resistance training and upper body exercise seem only promising. The effect size of each exercise mode was calculated and compared to the effect size of walking exercise. However, because of heterogeneity, no meta-analysis was performed on specific randomised controlled trials (RCTs) comparing the standard of supervised walking exercise to alternative exercise regimes.

Therefore, the question regarding which exercise mode gives the most beneficial results in walking distance, health-related quality of life, or both, in patients with IC remains to be answered. Previous Cochrane systematic reviews focused on the effect of exercise compared with usual care and the value of a supervised exercise programme in relation to non-supervised exercise.,19 This systematic review will determine the effect of alternative exercise modes by analysing randomised controlled trials comparing the current standard of supervised walking exercise to alternative modes of exercise. Studies focusing on this research topic are increasing, implicating the need for a regular update of this review in the upcoming years. 


\section{OBJECTIVES}

Primary objective: To assess the effects of different modes of supervised exercise therapy on the maximum walking distance (MWD) of patients with intermittent claudication.

Secondary objectives: To assess the effects of different modes of supervised exercise therapy on pain-free walking distance (PFWD) and health-related quality of life scores (HR-QoL) of patients with intermittent claudication.

\section{METHODS}

\section{Criteria for considering studies for this review \\ Types of studies}

We included parallel-group, randomised controlled trials (RCTs) comparing (combinations of) alternative modes of exercise training (for example, ergometry, strength training, aerobic exercise, etc.) with supervised walking exercise in patients with IC. We excluded crossover, factorial, or cluster RCTs.

\section{Types of participants}

The study population consisted of adults ( 18 years and older) with clinically determined IC, according to Fontaine stage II or Rutherford stages 1 to 3 , who were considered for conservative treatment. We excluded studies of participants with asymptomatic lower-limb atherosclerosis identified by testing. When studies described a mixture of asymptomatic and symptomatic participants, we contacted the authors to ask if a subanalysis was available. If not, we excluded these studies.

\section{Types of interventions}

We included all RCTs comparing alternative modes of exercise training (e.g. arm ergometry, strength training, cycling, etc.) or combinations of exercise modes with a control group of supervised walking exercise. Supervised walking exercise needed to be supervised at least twice a week for a consecutive six weeks of training. We excluded studies reporting an exercise programme with a duration of less than six weeks of training or with less than two supervised walking sessions a week.

Since different types of alternative exercise modes are associated with an increased walking capacity, we combined all studies with different alternative exercise modes in one analysis. However, to analyse the effect of each individual or combined alternative exercise modes, we performed a subgroup analysis on the exercise mode 
(or combination) if more than one study was available. We excluded all types of mechanical intermittent compression treatments as we did not consider them to be exercise training. Furthermore, we did not include studies comparing different types of walking exercise (supervised versus unsupervised, community versus hospital-based) or comparisons of different walking protocols (low- versus high-frequency training, low- versus high-intensity training, different treadmill exercise protocols).

\section{Types of outcome measures}

\section{Primary outcomes}

The primary outcome measurement was the maximum walking distance (MWD) measured by a treadmill test. Outcome measurements needed to be available at baseline and after at least six weeks of follow up. In case of different treadmill test protocols, we converted walking times or distances to total metabolic equivalents (total METs or sum of METs during the walking period).

\section{Secondary outcomes}

Secondary outcome measurements were the pain-free walking distance

(PFWD) and health-related quality of life (HR-QoL) scores. Besides a baseline measurement, results needed to be available after at least six weeks of follow up. In case of different treadmill test protocols, we converted walking times or distances to METs.

\section{Search methods for identification of studies}

\section{Electronic searches}

We did not use language restrictions. The Cochrane Peripheral Vascular Diseases Group Trials Search Coordinator (TSC) searched the Cochrane Peripheral Vascular Diseases Group Specialised Register (last searched July 2013) and the Cochrane Central Register of Controlled Trials (CENTRAL) 2013, Issue 6, part of The Cochrane Library, www.thecochranelibrary.com. The Specialised Register is maintained by the TSC and is constructed from weekly electronic searches of MEDLINE, EMBASE, CINAHL, AMED, and through handsearching relevant journals. The full list of the databases, journals and conference proceedings which have been searched, as well as the search strategies used are described in the Specialised Register section of the Cochrane Peripheral Vascular Diseases Group module in The Cochrane Library www. thecochranelibrary.com. 
The TSC searched the following trial databases (July 2013) for details of ongoing and unpublished studies using the terms exercise and claudication:

- The World Health Organization International Clinical Trials Registry (ICTRP) platform (apps.who.int/trialsearch/).

- The US National Institutes of Health Ongoing Trials Register (www.clinicaltrials.gov).

- The metaRegister of Controlled Trials (www.controlledtrials.com/isrctn/).

\section{Searching other resources}

The authors searched MEDLINE (1946 to 15 July 2013) and Embase (1973 to 15 July 2013). We reviewed the reference lists of articles identified by the above search strategies to identify other relevant citations.

\section{Data collection and analysis}

\section{Selection of studies}

GJL and FF independently selected trials for this review. SS and JT confirmed the suitability of selected trials for inclusion in the review. We sought additional information for included trials, if necessary.

\section{Data extraction and management}

GJL and FF independently extracted data using a standard data collection form created for this review. We entered the data into Review Manager (RevMan 5.2). We resolved disagreements between the review authors by discussion. SS acted as arbiter if no consensus was achievable. The extracted study data consisted of the following:

1. study characteristics, including study design, method of randomisation, exclusions postrandomisation, publication year, country, and study period;

2. baseline characteristics, including number of participants, losses to follow up, mean age, gender distribution, and inclusion and exclusion criteria;

3. type of interventions, including mode(s) of exercise, duration of programme, number of sessions, number of supervised sessions, and exercise protocol; and

4. the mean maximum walking distance or time, mean painfree walking distance or time, and mean quality of life scores at baseline and follow-up periods.

\section{Assessment of risk of bias in included studies}

The authors (GJL and FF) assessed the risk of bias for each study as described in the Cochrane Handbook for Systematic Reviews of Interventions ${ }^{20}$ for each of the following domains: 
1. randomisation/sequence generation;

2. allocation concealment;

3. blinding (of participants, personnel, and outcome assessors);

4. incomplete outcome data;

5. selective outcome reporting; and

6. publication and other sources of bias.

\section{Measures of treatment effect}

To analyse treatment effect, we assessed the MWD, PFWD, and HR-QoL scores after participation in the exercise programme. In the case of different treadmill test protocols, we converted walking times or distances to total METs (sum of METs during the walking period) using the American College of Sports Medicine (ACSM) walking equation. ${ }^{21}$

The ACSM walking equation includes the time, speed, and inclination of the treadmill test. Since direct conversion of the walking times or distances to METs was not possible because of the absence of individual participant data, we simulated a new dataset for each study. We used post intervention walking distances and variances from the intervention and control group to simulate a new dataset assuming a normal distribution. For each simulated individual, we calculated the number of METs using the ACSM walking equation. We used the summary measures (mean METs and variances) from each simulated dataset as post intervention outcomes from the included studies.

\section{Unit of analysis issues}

We searched for RCTs with at least six weeks' duration of training and a parallel-group design. We included no crossover trials in this review. In assessment of the primary and secondary outcomes, we considered the participant the unit of analysis.

\section{Dealing with missing data}

We expected missing standard deviations for walking distances. In the case of missing data, we requested data from the original investigators, if appropriate. We did not impute missing outcome data for the primary and secondary outcomes.

\section{Assessment of heterogeneity}

For all the outcome measures, we assessed statistical heterogeneity by calculating the $Q$-statistic or $\mathrm{Chi}^{2}$ test $\left(\mathrm{P}<0.10\right.$ considered as heterogeneous) and the $\mathrm{I}^{2}$ statistic $\left(\mathrm{I}^{2}\right.$ statistic greater than $50 \%$ considered as moderate to substantial risk of heterogeneity) in order to assess to what degree the data from the included studies were heterogeneous. ${ }^{20}$ 


\section{Assessment of reporting biases}

In case of sufficient studies (> 10 studies), we planned to assess publication bias with a funnel plot with the maximum walking distance on the $X$ axis and the standard error of each study on the $Y$ axis. ${ }^{20}$ If there is bias, for example, because smaller studies without statistically significant effects remain unpublished, this will lead to an asymmetrical appearance of the funnel plot. In this situation, the effect calculated by the meta-analysis will tend to overestimate the intervention effect. Therefore, we also planned

to evaluate funnel plot asymmetry using Begg and Egger tests 22,23 performed with Stata statistical software. ${ }^{24}$

\section{Data synthesis}

To analyse treatment effect, we used the DerSimonian and Laird random-effects model. This model takes the variance between studies and the variance within a study into account. ${ }^{25}$ We summarised the data of each study in forest plots and calculated summary estimates with a $95 \%$ confidence interval. We considered a two-sided $P$ $<0.05$ as statistically significant, except for the test of publication bias for which the recommended levels are $\mathrm{P}<0.10$. We performed analyses using RevMan 5.2.

\section{Subgroup analysis and investigation of heterogeneity}

We performed a subgroup analysis of each type of alternative exercise mode if we found more than one trial comparing the specific exercise mode with walking exercise. Furthermore, because most studies and international guidelines advise a 12-week supervised exercise programme, we reported outcomes both at the end of training and at 12 weeks of training. Finally, we performed a subgroup analysis on the combination of alternative exercise modes in relation to supervised walking exercise.

\section{Sensitivity analysis}

We examined individual study effects on the results by removing each study one at a time to examine whether removing a particular study would significantly change the results. In addition, we planned to perform sensitivity analyses on the methodological quality of the studies. We planned to exclude studies with apparent methodological flaws and risk of bias and examine whether removing these studies significantly changed results. 


\section{RESULTS}

Results of the search

FIGURE 1. Study flow diagram

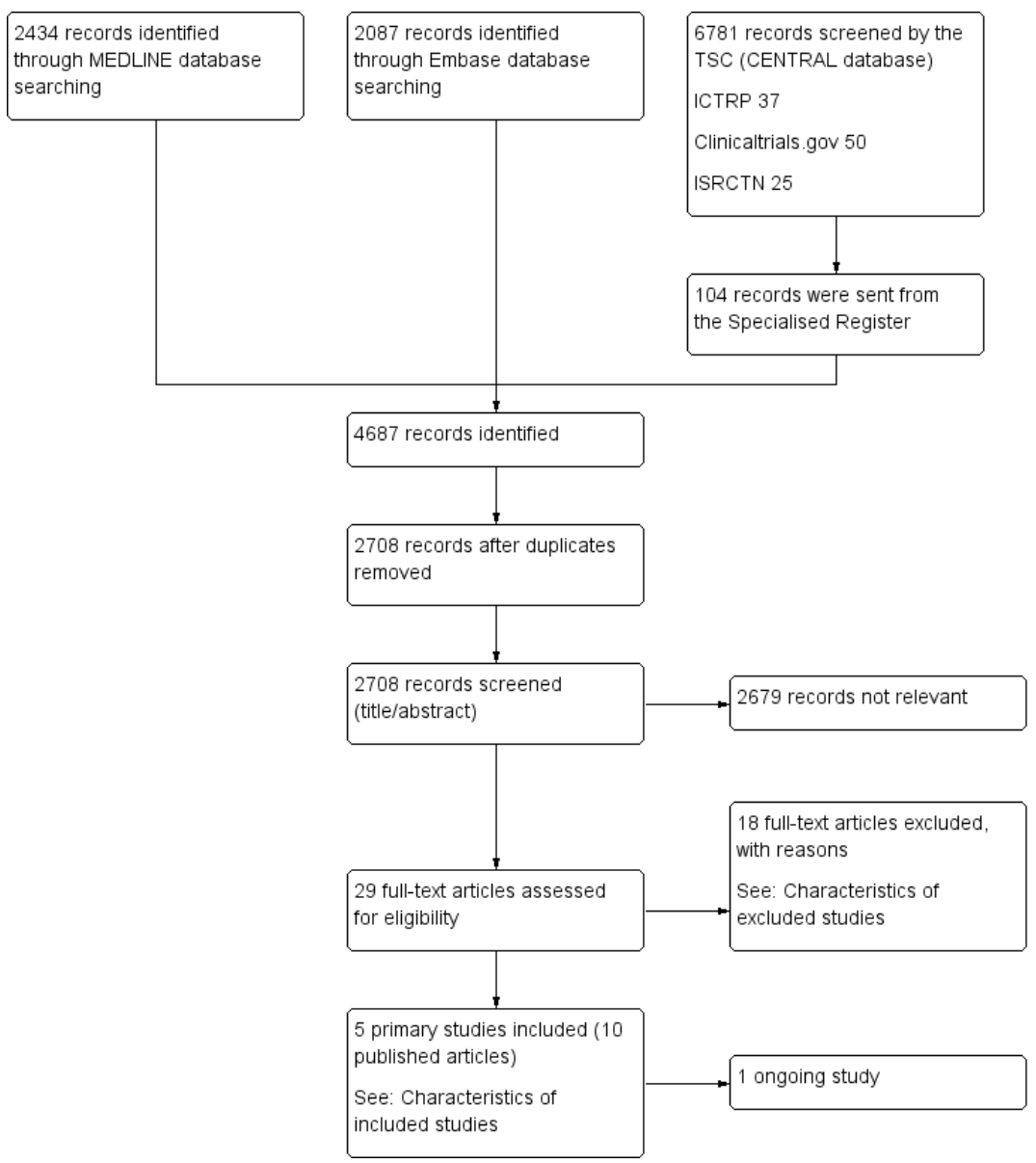

Included studies

We identified 10 publications comparing an alternative exercise regime to supervised walking exercise for IC. Of these 10 publications, we identified five primary studies. ${ }^{15,16,26-28}$ These five randomised controlled trials published in peer-reviewed journals fulfilled the inclusion criteria, and we considered these for inclusion in this review. Five additional publications described the results of three of the primary studies more extensively. ${ }^{29-33}$ 
Three trials compared supervised walking exercise to an exercise or progressive resistance regime ${ }^{26-28}$; one trial compared arm ergometry to supervised walking exercise ${ }^{16}$ and one trial compared cycling exercise to supervised walking exercise. ${ }^{15}$ These five trials randomised a total of 184 participants with IC, with 135 participants randomised to the treatment arms relevant to this review. The number of participants per study was small, with a variation of between 29 and 45 participants. The mean age of the participants in the included trials varied between 62.0 and 71.7 years, and all trials included both men and women. The trials were conducted in the United States (3), Brazil (1), and Australia (1).

Enrolment criteria were rather homogeneous. All trials included participants if a declination in ankle brachial index was present with coinciding limiting or disabling symptoms of IC. One trial ${ }^{26}$ assessed claudication symptoms by a questionnaire (San Diego Claudication Questionnaire). In all trials, the presence of critical limb ischaemia was an exclusion criterion. Participants were also excluded if the exercise capacity was limited by another factor than IC (e.g. angina, chronic obstructive pulmonary disease, arthrosis). In three of the five trials, claudication symptoms needed to be stable for, respectively, three months ${ }^{27}$ six months ${ }^{28}$, or more than 12 months. ${ }^{15}$ Two trials ${ }^{27,28}$ excluded participants if a revascularisation procedure was performed in the previous year. One RCT ${ }^{16}$ excluded participants if a coronary or lower-extremity revascularization procedure was performed within the past three months. The two remaining trials 26,15 excluded participants if they recently underwent surgery or a cardiovascular event. Treatment duration varied between studies ranging from six weeks' training ${ }^{15}, 12$-week training ${ }^{16,27,28}$, to 24 -week training periods. ${ }^{26}$

\section{Excluded studies}

After title/abstract screening, we excluded 3626 studies. After full text assessment, we excluded another 18 studies for various reasons. We excluded three studies as they were not randomised controlled trials. ${ }^{34-36}$ We excluded four studies ${ }^{37-40}$ as they were meeting posters with a limited description of the methods and results; no articles of these meeting posters were published yet. We excluded nine studies ${ }^{40-48}$ because they did not assess (adequate) supervised exercise therapy according to our inclusion criteria for this review. Five studies $41,43,44,46,49$ did not report the primary and secondary outcome measures of this review. We excluded one study ${ }^{50}$ because the outcome measures were unclearly described. We tried to contact the authors but did not receive additional information. We excluded one study ${ }^{51}$ because we did not consider the intervention (pole walking) an alternative exercise regime in comparison to supervised walking. 
Risk of bias in included studies

See Figure 2 for a summary of the risk of bias in each included study.

FIGURE 2. 'Risk of bias' summary: review authors' judgements about each 'Risk of bias' item for each included study

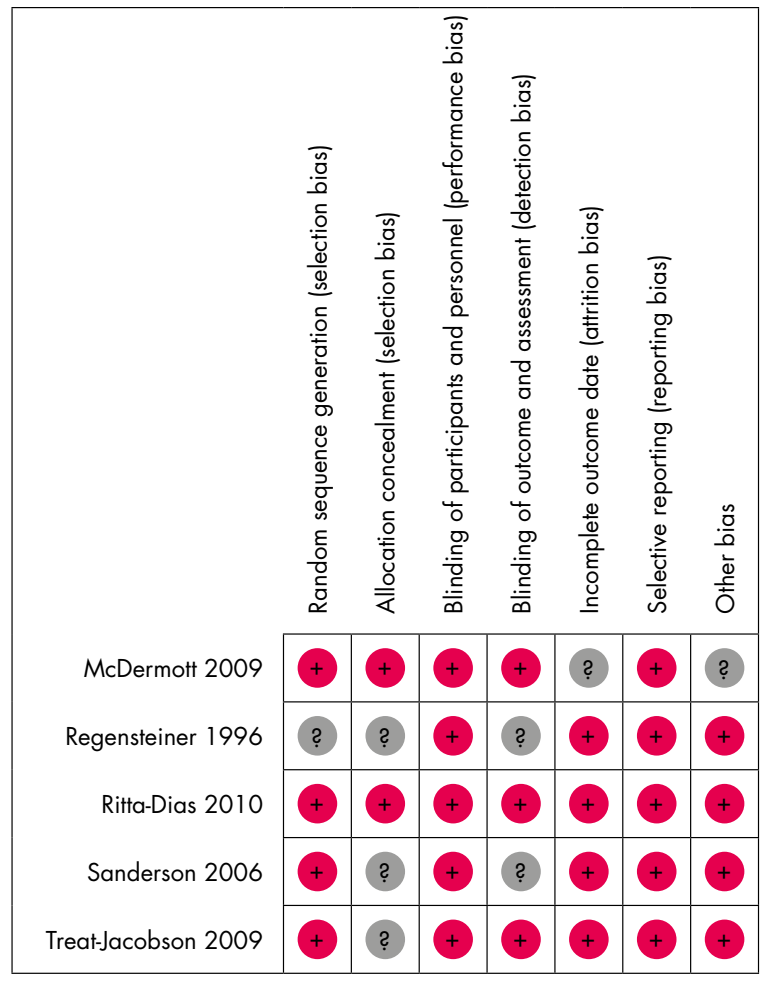

\section{Allocation}

Two studies described adequate sequence generation and allocation concealment by means of computer randomisation. ${ }^{26,28}$ Two studies an adequate sequence generation, but did not describe the allocation concealment. ${ }^{15,16}$ One study did not describe the randomisation process. ${ }^{27}$

\section{Blinding}

In the included studies, participants and direct personnel could not be blinded to the intervention (exercise). For this reason, bias could be introduced. However, since all studies experienced the same limitation, we considered the risk of bias to be low for all studies. Detection bias can be avoided by blinding the outcome assessors. Three studies achieved this. ${ }^{16,26,28}$ 


\section{Incomplete outcome data}

Most studies well-described reasons for missing data, and we considered these plausible and well distributed among the groups. One study addressed asymptomatic participants with PAOD as well as symptomatic participants. ${ }^{26}$ After contacting the authors, we derived the data of the sole symptomatic group with IC. However, it is unclear which of the missing data described in the article related to the symptomatic group. For this reason, we considered the incomplete outcome data in this study to be unclear.

\section{Selective reporting}

All studies described relevant outcomes. For three studies, we retrieved additional outcome data by contacting the authors. ${ }^{15,16,26}$

\section{Other potential sources of bias}

Since we identified only five studies, we could not detect publication bias with a funnel plot. Additional Begg and Egger tests did also exclude any indication of publication bias (Begg: adjusted Kendall's score $=4, \mathrm{P}=0.462$; Egger: bias 1.11, 95\% confidence interval $(\mathrm{Cl}) 0.43$ to $-2.77, \mathrm{P}=0.430$ ). One of the studies did not describe the baseline characteristics of the subgroup of participants with intermittent claudication. ${ }^{26}$ This was due to the study setting. (The study included participants with asymptomatic as well as symptomatic peripheral arterial disease.) However, we identified no other potential sources of bias in the included trials.

\section{Effects of interventions}

\section{Walking exercise versus alternative exercise \\ Maximum walking distance [METs]}

Data for the maximum walking distance (MWD) obtained at the end of each study were available in all of the included trials, with a total sample size of 135 participants. We calculated the pooled treatment effect after standardising the reported walking distances. For this reason, we converted all distances to metabolic equivalents (METs) using the American College of Sports Medicine (ACSM) formulas for metabolic calculations. We considered the impact of heterogeneity as low with an $I^{2}$ statistic of $13 \%$. At the end of training, the pooled MWD increased with an overall non-significant effect size of $8.15 \mathrm{METs}$ (95\% confidence interval $(\mathrm{Cl})-2.63$ to $18.94, \mathrm{P}=0.14$ ) in favour of walking exercise. This is the equivalent of an increase of 173 metres $195 \% \mathrm{Cl}$ -56 to 401 metres) on a treadmill with no incline and an average speed of $3.2 \mathrm{~km} / \mathrm{h}$, which is comparable with walking in daily life (Analysis 1.1). However, because of 
the width of the $\mathrm{Cl}$ of the pooled MWD, we could not identify any clear evidence of difference between interventions.

Furthermore, we calculated the effect size after 12 weeks of training. After this follow-up period, data from three trials on MWD were available with a sample size of 74 participants. ${ }^{16,27,28}$ We considered the impact of heterogeneity as moderate to substantial with an $\mathrm{I}^{2}$ statistic of $52 \%$. In these trials, the pooled MWD increased with a non-significant effect size of $13.05 \mathrm{METs}(95 \% \mathrm{Cl}-11.43$ to $37.54, \mathrm{P}=0.30)$ in favour of walking exercise. This correlates with 276 metres ( $95 \% \mathrm{Cl}-242$ to 795 metres) on a treadmill with no incline and an average speed of $3.2 \mathrm{~km} / \mathrm{h}$ (Analysis 1.2).

ANALYSIS 1.1. Comparison 1Walking exercise versus alternative exercise, Outcome 1 Maximum walking distance at the end of training.

\begin{tabular}{|c|c|c|c|c|c|c|c|}
\hline \multirow[t]{2}{*}{ Study or subgroup } & \multirow{2}{*}{ Walking exercise } & \multicolumn{2}{|r|}{ Alternative exercise } & \multicolumn{2}{|r|}{$\begin{array}{r}\text { Mean } \\
\text { Difference }\end{array}$} & \multirow[t]{2}{*}{ Weight } & $\begin{array}{r}\text { Mean } \\
\text { Difference }\end{array}$ \\
\hline & & Mean(SD)[METs] & $\mathrm{N}$ & Mean(SD)[METs] & IV,Random,95\% Cl & & IV,Random, $95 \% \mathrm{Cl}$ \\
\hline Ritta-Dias 2010 & 15 & $58.9(33.6)$ & 15 & $66.5(39.9)$ & 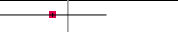 & $14.9 \%$ & $-7.60[-34.00,18.80]$ \\
\hline McDermott 2009 & 17 & $41.2(18.2)$ & 16 & $35.9(18.2)$ & - & $49.3 \%$ & $5.30[-7.12,17.72]$ \\
\hline Sanderson 2006 & 13 & $77.3(34.8)$ & 15 & $67.7(47.4)$ & & $11.5 \%$ & $9.60[-20.95,40.15]$ \\
\hline Treat-Jacobson 2009 & 13 & $66.2(38)$ & 12 & $51.2(24.9)$ & 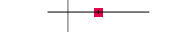 & $16.5 \%$ & $15.00[-10.00,40.00]$ \\
\hline Regensteiner 1996 & 10 & $73.5(53)$ & 9 & $33.8(27.7)$ & $\longrightarrow$ & $7.8 \%$ & $39.70[2.20,77.20]$ \\
\hline Total $(95 \% \mathrm{CI})$ & 68 & & 67 & & - & $100.0 \%$ & $8.15[-2.63,18.94]$ \\
\hline \multicolumn{8}{|c|}{ Heterogeneity: Tau $^{2}=21.21 ; \mathrm{Chi}^{2}=4.57, \mathrm{df}=4(\mathrm{P}=0.33) ; 1^{2}=13 \%$} \\
\hline \multicolumn{8}{|c|}{ Test for overall effect: $Z=1.48(P=0.14)$} \\
\hline \multicolumn{8}{|c|}{ Test for subgroup differences: Not applicable } \\
\hline & & & & -50 & -25 & & \\
\hline
\end{tabular}

\section{Pain-free walking distance [METs]}

Data for the pain-free walking distance (PFWD) obtained at the end of each study were available in four of the five included trials, with a total sample size of 116 participants. ${ }^{15,16,26-28}$ We calculated the effect after converting the reported walking distances to standardised METs. We considered the impact of heterogeneity as moderate with an $\mathrm{I}^{2}$ statistic of $49 \%$. At the end of training, the PFWD increased with an overall non-significant effect size of $6.42 \mathrm{METs}(95 \% \mathrm{Cl}-1.52$ to $14.36, \mathrm{P}=0.11)$ in favour of walking exercise. This is the equivalent of an increase of 136 metres $195 \% \mathrm{Cl}$ -32 to 304 metres) on a treadmill with no incline and an average speed of $3.2 \mathrm{~km} / \mathrm{h}$, which is comparable with walking in daily life (Analysis 1.3). However, because of the width of the $\mathrm{Cl}$ of the pooled PFWD, we could not identify any clear evidence of a difference between interventions. 
ANALYSIS 1.2. Comparison 1Walking exercise versus alternative exercise, Outcome 2 Maximum walking distance after 12 weeks of training.

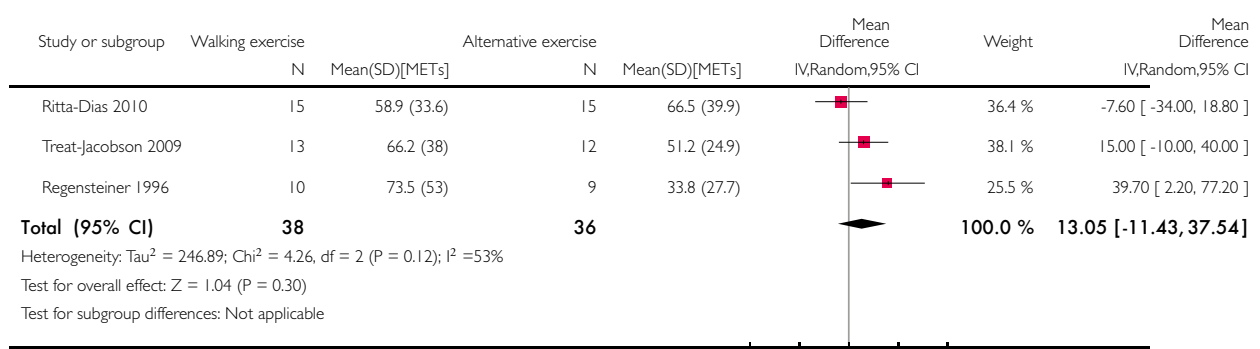

Furthermore, we calculated the effect size after 12 weeks of training. After this followup period, data for two trials for PFWD were available, with a sample size of 55 participants. ${ }^{16,28}$ We considered the impact of heterogeneity as low with an $I^{2}$ statistic of $0 \%$. In these trials, the pooled PFWD increased with a non-significant effect size of 2.37 METs (95\% Cl -5.81 to 10.56, $\mathrm{P}=0.57$ ) in favour of walking exercise. This correlates with 50 metres $(95 \% \mathrm{Cl}-123$ to 224 metres) on a treadmill with no incline and an average speed of $3.2 \mathrm{~km} / \mathrm{h}$ (Analysis 1.4).

\section{Health-related quality of life (HR-QoL) and functional impairment}

Two of the included studies described HR-QoL and functional impairment. ${ }^{26,27}$ One study used the Medical Outcomes Study (MOS) SF-20; another study used the SF-36 Physical Functioning score to collect HR-QoL data. ${ }^{26,27}$ Both studies used the Walking Impairment Questionnaire (WIQ) to describe functional impairment.

Data on HR-QoL and functional impairment (WIQ) from one study, with a total sample size of 19 participants, showed an increase in HR-QoL with a significant effect size of $26.50 \%(95 \% \mathrm{Cl} 2.67$ to $50.33, \mathrm{P}=0.03)$ in favour of walking exercise. ${ }^{27} \mathrm{WIQ}$ distance score increased with an effect size of $2.00 \% 195 \% \mathrm{Cl}-16.04$ to $20.04, \mathrm{P}=$ 0.83 ) in favour of walking exercise. WIQ speed score decreased with an effect size of $-4.50 \%(95 \% \mathrm{Cl}-27.34$ to $18.34, \mathrm{P}=0.70)$ in favour of alternative exercise. WIQ stair-climbing score decreased with an effect size of $-29.50 \%(95 \% \mathrm{Cl}-51.65$ to -7.35 , $P=0.009$ ) in favour of alternative exercise. Unfortunately, both the SF-36 Physical Functioning score and $\mathrm{WIQ}$ data from the second study were not normally distributed.

SF-36 Physical Functioning score improved in both the strength $(n=14)$ and treadmill walking group ( $\mathrm{n}=17$ ), with a median of, respectively, 12.5 points (interquartile range 
$=-5.00$ to 20.00 ) and 10.0 points (interquartile range $=5.00$ to 20.00 ), $P=0.811 .^{26}$ WIQ distance score improved in both the strength $(n=15)$ and treadmill walking group $(n=15)$, with a median of, respectively, 14.0 points (interquartile range = 1.56 to 26.6 ) and 7.46 points (interquartile range $=-0.36$ to 25.0 ), $P=0.431$. WIQ speed score improved in both the strength $(n=15)$ and treadmill walking group $(n=$ 16), with a median of, respectively, 3.26 points (interquartile range $=-7.61$ to 26.1 ) and 1.63 points (interquartile range $=-3.80$ to 28.8 ), $P=0.736$. WIQ stair-climbing score improved in the strength training group $(n=15)$, with a median of, respectively, 12.5 points (interquartile range $=4.17$ to 25.0 ), while we saw no improvement in the median score in the treadmill walking group $(n=16$, median score of 0.00 points, interquartile range $=0.00$ to 14.6 ),$P=0.136$. Because of the skewed data of one of the studies and the small sample size of both studies, we did not transform these data to perform a meta-analysis of the two studies. ${ }^{26}$

ANALYSIS 1.3. Comparison 1Walking exercise versus alternative exercise, Outcome 3 Pain-free walking distance at the end of training.

\begin{tabular}{|c|c|c|c|c|c|c|c|}
\hline \multirow[t]{2}{*}{ Study or subgroup } & Walking exercise & \multicolumn{2}{|r|}{ Alternative exercise } & \multirow[b]{2}{*}{ Mean(SD)[METs] } & \multirow{2}{*}{$\begin{array}{c}\text { Mean } \\
\text { Difference } \\
\text { IV,Random, } 95 \% \mathrm{Cl}\end{array}$} & \multirow[t]{2}{*}{ Weight } & \multirow{2}{*}{$\begin{array}{r}\text { Mean } \\
\text { Difference } \\
\text { IV,Random, } 95 \% \mathrm{Cl}\end{array}$} \\
\hline & $\mathrm{N}$ & Mean(SD)[METs] & $\mathrm{N}$ & & & & \\
\hline Ritta-Dias 2010 & 15 & $32.8(21.4)$ & 15 & $37(26.4)$ & $\longrightarrow$ & $15.1 \%$ & $-4.20[-21.40,13.00]$ \\
\hline Treat-Jacobson 2009 & 13 & $16.8(15.4)$ & 12 & $12.5(7.2)$ & - & $30.4 \%$ & $4.30[-5.01,13.61]$ \\
\hline McDermott 2009 & 17 & $18.8(15.4)$ & 16 & $14.4(9.4)$ & $\mp$ & $32.3 \%$ & $4.40[-4.25,13.05]$ \\
\hline Sanderson 2006 & 13 & $31.6(22.8)$ & 15 & $12.1(6.2)$ & $\longrightarrow$ & $22.2 \%$ & $19.50[6.72,32.28]$ \\
\hline Total $(95 \% \mathrm{Cl})$ & 58 & & 58 & & - & $100.0 \%$ & $6.42[-1.52,14.36]$ \\
\hline \multicolumn{8}{|c|}{ Heterogeneity: $\mathrm{Tau}^{2}=31.43 ; \mathrm{Chi}^{2}=5.89, \mathrm{df}=3(\mathrm{P}=0.12) ; 1^{2}=49 \%$} \\
\hline \multicolumn{8}{|c|}{ Test for overall effect: $Z=1.58(P=0.11)$} \\
\hline \multicolumn{8}{|c|}{ Test for subgroup differences: Not applicable } \\
\hline & & & & -50 & 25 & 50 & \\
\hline & & & & Favours & Favours $V$ & Valking & \\
\hline
\end{tabular}

ANALYSIS 1.4. Comparison 1Walking exercise versus alternative exercise, Outcome 4 Pain-free walking distance after 12 weeks of training.

\begin{tabular}{|c|c|c|c|c|c|c|c|}
\hline \multirow[t]{2}{*}{ Study or subgroup } & Walking exercise & \multicolumn{2}{|r|}{ Alternative exercise } & \multicolumn{2}{|r|}{$\begin{array}{r}\text { Mean } \\
\text { Difference }\end{array}$} & \multirow[t]{2}{*}{ Weight } & \multirow{2}{*}{$\begin{array}{r}\text { Mean } \\
\text { Difference } \\
\text { IV,Random,95\% C }\end{array}$} \\
\hline & $N$ & Mean(SD)[METs] & $N$ & $\operatorname{Mean}(\mathrm{SD})[\mathrm{MET}$ s] & IV,Random,95\% Cl & & \\
\hline Ritta-Dias 2010 & 15 & $32.8(21.4)$ & 15 & $37(26.4)$ & 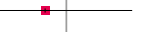 & $22.7 \%$ & $-4.20[-21.40,13.00]$ \\
\hline Treat-Jacobson 2009 & 13 & $16.8(15.4)$ & 12 & $12.5(7.2)$ & + & $77.3 \%$ & $4.30[-5.01,|3.6|]$ \\
\hline Total $(95 \% \mathrm{Cl})$ & 28 & & 27 & & 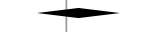 & $100.0 \%$ & $2.37[-5.81,10.56]$ \\
\hline \multicolumn{8}{|c|}{ Heterogeneity: $\operatorname{Tau}^{2}=0.0 ; \mathrm{Chi}^{2}=0.73, \mathrm{df}=\mathrm{I}(\mathrm{P}=0.39) ; \mathrm{I}^{2}=0.0 \%$} \\
\hline \multicolumn{8}{|c|}{ Test for overall effect: $Z=0.57(P=0.57)$} \\
\hline Test for subgroup differe & ences: Not applicab & & & & & & \\
\hline
\end{tabular}




\section{Sensitivity analysis}

We performed a sensitivity analysis to assess whether excluding an individual study would significantly change the main results on MWD and PFWD at the end of the study. For MWD, removing any individual study did not alter the results significantly, although by removing one study, the MWD increased almost significantly, in favour of walking exercise, with an overall effect size of $10.16 \mathrm{METs}(95 \% \mathrm{Cl}-0.40$ to $20.71, \mathrm{P}=0.06) .{ }^{28}$ This is the equivalent of an increase of 215 metres $(95 \% \mathrm{Cl}-8$ to 439 metres) on a treadmill with no incline and an average speed of $3.2 \mathrm{~km} / \mathrm{h}$.

For PFWD, removing one of the studies did not alter the results significantly, although by removing one study ${ }^{28}$, the PFWD increased almost significantly in favour of walking exercise, with an overall effect size of $8.30 \mathrm{METs}(95 \% \mathrm{Cl}-0.26$ to $16.86, \mathrm{P}=0.06)$. This is the equivalent of an increase of 176 metres $(95 \% \mathrm{Cl}-6$ to 357 metres) on a treadmill with no incline and an average speed of $3.2 \mathrm{~km} / \mathrm{h}$. We did not perform a sensitivity analysis on the methodological quality of the studies because of the limited number of studies.

\section{Walking exercise versus strength training}

\section{Maximum walking distance [METs]}

Data for MWD obtained at the end of each study were available in three of the five included trials, with a total sample size of 82 participants. ${ }^{26-28}$ We considered the impact of heterogeneity as moderate to substantial with an $\mathrm{I}^{2}$ statistic of $52 \%$. At the end of the study, the pooled MWD increased with an overall non-significant effect size of $8.11 \mathrm{METs}(95 \% \mathrm{Cl}-11.68$ to $27.90, \mathrm{P}=0.42)$ in favour of walking exercise. This is the equivalent of an increase of 172 metres $(95 \% \mathrm{Cl}-247$ to 591 metres) on a treadmill with no incline and an average speed of $3.2 \mathrm{~km} / \mathrm{h}$, which is comparable with walking in daily life (Analysis 2.1).

Furthermore, we calculated the effect size after 12 weeks of training. After this followup period, data for two trials for MWD were available, with a sample size of 49 participants. ${ }^{27,28}$ We considered the impact of heterogeneity as substantial with an $1^{2}$ statistic of $76 \%$. The pooled MWD increased with a non-significant effect size of 14.10 METs $(95 \% \mathrm{Cl}-32.10$ to $60.29, \mathrm{P}=0.55)$ in favour of walking exercise. This correlates with 299 metres (95\% Cl-680 to 1277 metres) on a treadmill with no incline and an average speed of $3.2 \mathrm{~km} / \mathrm{h}$ (Analysis 2.2). 
ANALYSIS 2.1. Comparison 2 Walking exercise versus strength training, Outcome 1 Maximum walking distance at the end of training.

\begin{tabular}{|c|c|c|c|c|c|c|c|}
\hline \multirow[t]{2}{*}{ Study or subgroup } & Walking exercise & \multicolumn{2}{|r|}{ Strength training } & & $\begin{array}{r}\text { Mean } \\
\text { Difference }\end{array}$ & \multirow[t]{2}{*}{ Weight } & \multirow{2}{*}{$\begin{array}{r}\text { Mean } \\
\text { Difference } \\
\text { IV,Random, } 95 \% \mathrm{Cl}\end{array}$} \\
\hline & $N$ & Mean(SD)[METs] & $\mathrm{N}$ & Mean(SD)[METs] & IV,Random, $95 \% \mathrm{Cl}$ & & \\
\hline Ritta-Dias 2010 & 15 & $58.9(33.6)$ & 15 & $66.5(39.9)$ & - & $29.8 \%$ & $-7.60[-34.00,18.80]$ \\
\hline McDermott 2009 & 17 & $41.2(18.2)$ & 16 & $35.9(18.2)$ & \# & $50.8 \%$ & $5.30[-7.12,17.72]$ \\
\hline Regensteiner 1996 & 10 & $73.5(53)$ & 9 & $33.8(27.7)$ & $\square$ & $19.4 \%$ & $39.70[2.20,77.20]$ \\
\hline Total $(95 \% \mathrm{Cl})$ & 42 & & 40 & & & $100.0 \%$ & $8.11[-11.68,27.90]$ \\
\hline \multicolumn{8}{|c|}{ Heterogeneity: $\operatorname{Tau}^{2}=160.49 ; \mathrm{Chi}^{2}=4.13, \mathrm{df}=2(P=0.13) ;\left.\right|^{2}=52 \%$} \\
\hline \multicolumn{8}{|c|}{ Test for overall effect: $Z=0.80(P=0.42)$} \\
\hline \multicolumn{8}{|c|}{ Test for subgroup differences: Not applicable } \\
\hline & & & & -100 & -50 & 100 & \\
\hline & & & & Favours & Favours $\mathrm{V}$ & Valking & \\
\hline
\end{tabular}

ANALYSIS 2.2. Comparison 2 Walking exercise versus strength training, Outcome 2 Maximum walking distance at 12 weeks of training.

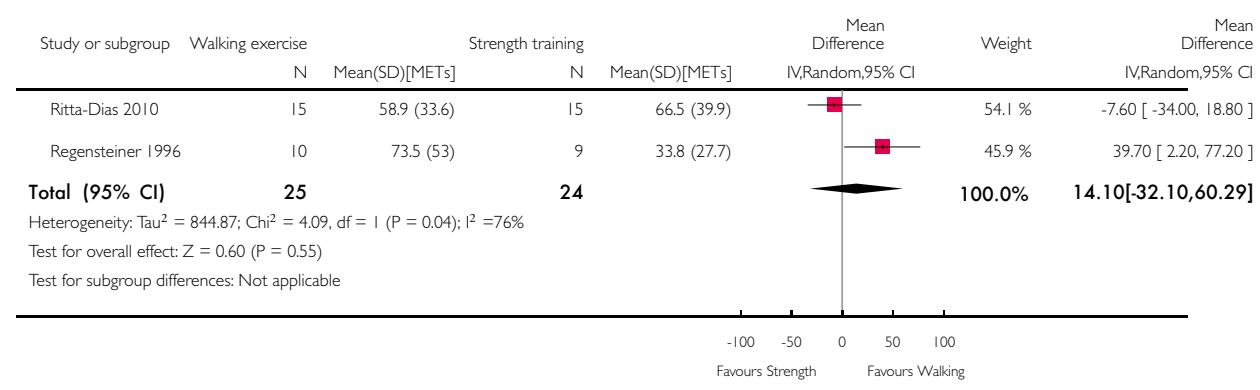

ANALYSIS 2.3. Comparison 2 Walking exercise versus strength training, Outcome 3 Pain-free walking distance at the end of training.

\begin{tabular}{|c|c|c|c|c|c|c|c|}
\hline \multirow[t]{2}{*}{ Study or subgroup } & Walking exercise & & Strength training & & $\begin{array}{r}\text { Mean } \\
\text { Difference }\end{array}$ & \multirow[t]{2}{*}{ Weight } & \multirow{2}{*}{$\begin{array}{r}\text { Mean } \\
\text { Difference } \\
\text { IV,Random, } 95 \% \mathrm{Cl}\end{array}$} \\
\hline & $\mathrm{N}$ & Mean(SD)[METs] & $\mathrm{N}$ & Mean(SD)[METs] & IV,Random,95\% Cl & & \\
\hline Ritta-Dias 2010 & 15 & $32.8(21.4)$ & 15 & $37(26.4)$ & $\Psi$ & $20.2 \%$ & $-4.20[-21.40,13.00]$ \\
\hline McDermott 2009 & 17 & $18.8(15.4)$ & 16 & $14.4(9.4)$ & i & $79.8 \%$ & $4.40[-4.25,13.05]$ \\
\hline Total $(95 \% \mathrm{Cl})$ & 32 & & 31 & & & $100.0 \%$ & $2.66[-5.06,10.39]$ \\
\hline \multicolumn{8}{|c|}{ Heterogeneity: $\operatorname{Tau}^{2}=0.0 ; \mathrm{Chi}^{2}=0.77, \mathrm{df}=\mathrm{I}(P=0.38) ; \mathrm{I}^{2}=0.0 \%$} \\
\hline \multicolumn{8}{|c|}{ Test for overall effect: $Z=0.68(P=0.50)$} \\
\hline \multicolumn{8}{|c|}{ Test for subgroup differences: Not applicable } \\
\hline & & & & & $\begin{array}{lll}-10 & 0 & 10\end{array}$ & 20 & \\
\hline \multicolumn{8}{|c|}{ Favours Strength $\quad$ Favours Walking } \\
\hline
\end{tabular}




\section{Pain-free walking distance [METs]}

Data for the PFWD at the end of the study were available in two of the included trials, with a total sample size of 63 participants. ${ }^{26,28}$ We considered the impact of heterogeneity as low with an $1^{2}$ statistic of $0 \%$. At the end of the study, the pooled PFWD increased with an overall non-significant effect size of $2.66 \mathrm{METs}(95 \% \mathrm{Cl}-5.06$ to $10.39, \mathrm{P}=0.50$ ) in favour of walking exercise. This is the equivalent of an increase of 56 metres ( $95 \% \mathrm{Cl}-107$ to 220 metres) on a treadmill with no incline and an average speed of $3.2 \mathrm{~km} / \mathrm{h}$, which is comparable with walking in daily life (Analysis 2.3).

\section{Walking exercise versus a combination of exercise modes}

\section{Maximum walking distance [METs]}

Two trials analysed the effect of a combination of exercise modes in relation to supervised walking exercise on the MWD. ${ }^{16,27}$ One trial compared a combination of walking exercise and arm ergometry to sole walking exercise. ${ }^{16}$ The second trial compared a combination of walking exercise and strength training to sole walking exercise. ${ }^{27}$ In total, the two trials had a sample size of 41 participants for analysis of the MWD at the end of the studies. We considered the impact of heterogeneity as low with an $I^{2}$ statistic of $0 \%$. The pooled MWD increased with an overall non-significant effect size of $9.31 \mathrm{METs}(95 \% \mathrm{Cl}-12.77$ to $31.40, \mathrm{P}=0.41)$ in favour of sole walking exercise. This is the equivalent of an increase of 197 metres $(95 \% \mathrm{Cl}-270$ to 665 metres) on a treadmill with no incline and an average speed of $3.2 \mathrm{~km} / \mathrm{h}$, which is comparable with walking in daily life (Analysis 3.1).

\section{Pain-free walking distance [METs]}

Data for PFWD obtained at the end of each study were available in one of the included trials with a total sample size of 25 participants. ${ }^{16}$ At the end of this study, the PFWD increased with an overall non-significant effect size of $3.30 \mathrm{METs}(95 \% \mathrm{Cl}-6.01$ to $12.61, P=0.49$ ) in favour of sole walking exercise. This is the equivalent of an increase of 70 metres ( $95 \% \mathrm{Cl}-127$ to 267 metres) on a treadmill with no incline and an average speed of $3.2 \mathrm{~km} / \mathrm{h}$, which is comparable with walking in daily life. 


\section{DISCUSSION}

\section{Summary of main results}

We included five randomised controlled trials (RCTs) in this review, with a total of 135 participants. We could not find any clear evidence of a statistical difference in maximum or pain-free walking distance when comparing supervised treadmill walking with an alternative exercise regime. Furthermore, sensitivity analysis did not significantly alter the results. Regarding quality of life, two studies described health related quality of life and functional impairment. ${ }^{26,27}$ However, the data in one of the two studies were not normally distributed. ${ }^{26}$ Therefore, we could not make any meaningful comparison between studies.

\section{Overall completeness and applicability of evidence}

Although the topic of this review is contemporary ${ }^{18}$, this review identified only five $R C T s$, with a total of 135 participants. The five RCTs described only three alternative exercise modes: strength training ${ }^{26-28}$, arm-ergometry ${ }^{16}$, and cycling exercise ${ }^{15}$. In total, three of the five studies ${ }^{26-28}$ compared strength training to supervised treadmill walking, for which we performed a subanalysis. Eventually, more studies are needed to make meaningful comparisons between each alternative exercise mode and the current standard of supervised treadmill walking. Therefore, the applicability of the current evidence is limited.

\section{Quality of the evidence}

The risk of bias of the included studies was, in general, low (see Figure 2), reflecting good methodological quality of the included studies. We could not detect publication bias because we could not assess asymmetry in a funnel plot with the limited number of studies. The quality of this review is however limited because of the small sample size of 135 participants. At analysis, we experienced some heterogeneity in Analysis 1.2, Analysis 2.1, and Analysis 2.2 using the $\mathrm{I}^{2}$ statistic and in Analysis 2.2 using the $\mathrm{Q}$-statistic or $\mathrm{Chi}^{2}$ test. Sensitivity analysis did not significantly alter the results of the review.

\section{Potential biases in the review process}

We tried to limit all potential biases in the review process. To limit bias and make a meaningful comparison, we standardised maximum walking distance (MWD) and pain-free walking distance (PFWD) from each study by converting walking distances to total metabolic equivalents (METs) according to the American College of Sports 
Medicine (ACSM) formulas for metabolic calculations. ${ }^{21}$ However, direct conversion of the walking times or distances to METs was not possible due to the absence of individual participant data. We therefore simulated a new dataset for each study to make a meaningful comparison. It is unclear to what extent this could have biased our findings.

We excluded one study solely because it did not report the correct outcome measures. ${ }^{49}$ Although we were careful to ascertain that relevant outcomes were not available because they were not measured rather than not reported, this could be a potential bias in the review process. In future updates, we will pay further attention to this potential source of bias.

\section{Agreements and disagreements with other studies or reviews}

We agree with a previous published systematic review ${ }^{18}$ that there was no clear evidence of difference between alternative exercise modes and supervised walking exercise for intermittent claudication. Results seem promising, but additional studies are urgently needed to validate these exercise modes in relation to the standard of supervised walking exercise.

\section{AUTHORS' CONCLUSIONS}

\section{Implications for practice}

Few studies were found and there was no clear evidence of a difference between supervised walking exercise and alternative exercise modes. More studies with larger sample sizes are needed to make meaningful comparisons between each alternative exercise mode and the current standard of supervised treadmill walking. The results indicate that alternative exercise modes may be useful when supervised walking exercise is not an option for the patient.

\section{Implications for research}

More studies are urgently needed to make meaningful comparisons between each alternative exercise mode and the current standard of supervised treadmill walking. 


\section{REFERENCES}

1. Dumville JC, Lee AJ, Smith FB, Fowkes FG The health related quality of life of people with peripheral arterial disease in the community: the Edinburgh Artery Study. British Journal of General Practice 2004;54(508):826-31.

2. Kannel WB, McGee DL. Update on some epidemiologic features of intermittent claudication: the Framingham Study. Journal of the American Geriatrics Society 1985;33(1):138.

3. McDermott MM, Greenland P, Liu K, Guralnik $J M$, Criqui $M H$, Dolan NC, et al.Leg symptoms in peripheral arterial disease: associated clinical characteristics and functional impairment. JAMA 2001 ;286(13):1599-606.

4. Hirsch AT, Haskal ZJ, Hertzer NR, Bakal CW, Creager MA, Halperin JL, et al.ACC/AHA 2005 Practice guidelines for the management of patients with peripheral arterial disease (lower extremity, renal, mesenteric, and abdominal aortic): a collaborative report from the American Association for Vascular Surgery/ Society for Vascular Surgery, Society for Cardiovascular Angiography and Interventions, Society for Vascular Medicine and Biology, Society of Interventional Radiology, and the ACC/AHA Task Force on Practice Guidelines (Writing Committee to Develop Guidelines for the Management of Patients With Peripheral Arterial Disease): endorsed by the American Association of Cardiovascular and Pulmonary Rehabilitation; National Heart, Lung, and Blood Institute; Society for Vascular Nursing; TransAtlantic Inter-Society Consensus; and Vascular Disease Foundation. Circulation 2006;113(11): e463-e654.

5. Norgren L, Hiatt WR, Dormandy JA, Nehler MR, Harris KA, Fowkes FG, TASC II Working Group. Inter-society consensus for the management of peripheral arterial disease (TASC II). Journal of Vascular Surgery 2007;45 Suppl S: S5-67.

6. Creasy TS, McMillan PJ, Fletcher EW, Collin J, Morris PJ. Is percutaneous transluminal angioplasty better than exercise for claudication? Preliminary results from a prospective randomised trial. European Journal of Vascular Surgery 1990;4(2):135-40.

7. Fokkenrood HJP, Bendermacher BLW, Lauret GJ, Willigendael EM, Prins MH, Teijink JAW. Supervised exercise therapy versus nonsupervised exercise therapy for intermittent claudication. Cochrane Database of Systematic Reviews 2013, Issue 8.
8. Fowkes FG, Gillespie IN. Angioplasty (versus non surgical management) for intermittent claudication. Cochrane Database of Systematic Reviews 1998, Issue 2.

9. Lundgren F, Dahllöf AG, Lundholm K, Scherstén $\mathrm{T}$, Volkmann R. Intermittent claudicationsurgical reconstruction or physical training? A prospective randomized trial of treatment efficiency. Annals of Surgery 1989;209(3):34655.

10. Spronk S, Bosch JL, den Hoed PT, Veen HF, Pattynama PM, Hunink MG. Intermittent claudication: clinical effectiveness of endovascular revascularization versus supervised hospital-based exercise trainingrandomized controlled trial. Radiology $2009 ; 250(2): 586-95$.

11. Bendermacher BL,Willigendael EM, Nicolaï SP, Kruidenier LM, Welten RJ, Hendriks E, et al. Supervised exercise therapy for intermittent claudication in a community-based setting is as effective as clinic-based. Journal of Vascular Surgery 2007;45(6): 1192-6.

12. Kruidenier LM, Nicolai SP, Hendriks EJ Bollen EC, Prins MH, Teijink JA. Supervised exercise therapy for intermittent claudication in daily practice. Journal of Vascular Surgery 2009;49(2):363-70.

13. Nicolaï SP, Teijink JA, Prins MH, Exercise Therapy in Peripheral Arterial Disease Study Group. Multicenter randomized clinical trial of supervised exercise therapy with or without feedback versus walking advice for intermittent claudication. Journal of Vascular Surgery 2010;52(2):348-55.

14. Hiatt WR, Wolfel EE, Meier RH, Regensteiner JG. Superiority of treadmill walking exercise versus strength training for patients with peripheral arterial disease. Implications for the mechanism of the training response. Circulation 1994;90(4):1866-74.

15. Sanderson B, Askew C, Stewart I, Walker P, Gibbs H, Green S. Short-term effects of cycle and treadmill training on exercise tolerance in peripheral arterial disease. Journal of Vascular Surgery 2006;44(1):119-27.

16. Treat-Jacobson D, Bronas UG, Leon AS Efficacy of arm-ergometry versus treadmill exercise training to improve walking distance in patients with claudication. Vascular Medicine 2009; 14(3):203-13. 
17. Hamburg NM, Balady GJ. Exercise rehabilitation in peripheral artery disease: functional impact and mechanisms of benefits. Circulation $2011 ; 123(1): 87-97$.

18. Parmenter BJ, Raymond J, Dinnen P, Singh MA. A systematic review of randomized controlled trials: walking versus alternative exercise prescription as treatment for intermittent claudication. Atherosclerosis $2011 ; 218(1): 1-$ 12.

19. Watson L, Ellis B, Leng GC. Exercise for intermittent claudication. Cochrane Database of Systematic Reviews 2008, Issue 4.

20. Higgins JPT, Green S (editors). Cochrane Handbook for Systematic Reviews of Interventions Version 5.1.0 [Updated March 2011 ]. The Cochrane Collaboration, 2011.

21. American College of Sports Medicine (ACSM). ACSM'S Metabolic Calculations Handbook. Lippincott Williams \& Wilkins, 2006.

22. Begg $\quad C B$, Mazumdar M. Operating characteristics of a rank correlation test for publication bias. Biometrics 1994;50(4): 1088101.

23. Egger M, Davey Smith G, Schneider M, Minder $C$. Bias in meta-analysis detected by a simple, graphical test. BMJ 1997;315(7109):629-34.

24. StataCorp LP. Stata Statistical Software. 12. College Station, Texas: StataCorp LP, 2011.

25. DerSimonian R, Laird N. Meta-analysis in clinical trials. Controlled Clinical Trials 1986;7(3):177-88.

26. McDermott MM, Ades P, Guralnik JM, Dyer A, Ferrucci L, Liu K, et al.Treadmill exercise and resistance training in patients with peripheral arterial disease with and without intermittent claudication: a randomized controlled trial. JAMA 2009;301(2):165-74.

27. Regensteiner JG, Steiner JF, Hiatt WR. Exercise training improves functional status in patients with peripheral arterial disease. Journal of Vascular Surgery 1996;23(1):104-15.

28. Ritta-Dias RM, Wolosker N, de Moraes Forjaz CL, Carvalho CR, Cucato GG, Leão $P P$, et al.Strength training increases walking tolerance in intermittent claudication patients: randomized trial. Journal of Vascular Surgery 2010; 51(1):89-95.
29. Hiatt WR, Regensteiner JG, Wolfel EE, Carry $M R$, Brass EP. Effect of exercise training on skeletal muscle histology and metabolism in peripheral arterial disease. Journal of Applied Physiology 1996;81(2):780-8.

30. Hiatt WR, Wolfel EE, Meier RH, Regensteiner JG. Superiority of treadmill walking exercise versus strength training for patients with peripheral arterial disease. Implications for the mechanism of the training response. Circulation 1994;90(4): 1866-74.

31. Grizzo Cucato G, de Moraes Forjaz CL, Kanegusuku $H$, da Rocha Chehuen $M$, Riani Costa LA, Wolosker N, et al.Effects of walking and strength training on resting and exercise cardiovascular responses in patients with intermittent claudication. Vasa $2011 ; 40(5): 390-7$.

32. Menêses $A L$, de Lima $G H$, Forjaz $C L$, Lima $A H$, Silva $G Q$, Cucato $G G$, et al.Impact of a supervised strength training or walking training over a subsequent unsupervised therapy period on walking capacity in patients with claudication. Journal of Vascular Nursing $2011 ; 29(2): 81-6$.

33. Bronas UG, Treat-Jacobson D, Leon AS. Comparison of the effect of upper body ergometry aerobic training vs treadmill training on central cardiorespiratory improvement and walking distance in patients with claudication. Journal of Vascular Surgery $2011 ; 53(6)$ :155764.

34. Gardner AW. Supervised exercise therapy provided by local physiotherapists improves walking distance in patients with claudication. Evidence-based Medicine $2011 ; 16(2): 43-4$.

35. Kim DH. Exercise and peripheral arterial disease. Annals of Internal Medicine 2006; 144(9):author reply 699-700.

36. Roitman JL. Treadmill exercise and resistance training in patients with peripheral arterial disease with and without in intermittent claudication: a randomized controlled trial. Journal of Cardiopulmonary Rehabilitation and Prevention 2010;30(1):62.

37. Dedes H, Figoni SF, Kalioundii G, Kunkel C, Peter A, Phillips $A$, et al.Prospective trial of calf ergometry training on walking ability in peripheral arterial disease. PM\&R 2010;219 Suppl 1):S26. 
38. Kuwabara M, Anzai H, Nishi Y, Hayashida N, Hisatome I. Treadmill and ergometer exercise once a week equally improve not only exercisetolerance but also cardio-renal function in peripheral arterial disease (PAD) patients with claudication. Journal of the American College of Cardiology 2010;55(10s1):E1479-A158.

39. Ornelas CC, Dhingsa K, Etnyre G, Figoni SF, Fung DA, Kunkel C, et al.Comparison of calf ergometer and treadmill exercise training interventions for participants with intermittent claudication due to peripheral arterial disease. PM\&R 2011;3(10 Suppl 1):S266-7.

40. Treat-Jacobson D, Bronas UG, Krause BJ, Robinson CA, Santilli SM, Leon AS. Aerobic arm exercise training to improve outcomes for patients with severe claudication and ischemic rest pain. Vascular Medicine 2012;17(3):204.

41. Nawaz S, Walker RD, Wilkinson CH, Saxton JM, Pockley AG, Wood RF. The inflammatory response to upper and lower limb exercise and the effects of exercise training in patients with claudication. Journal of Vascular Surgery 2001; 33(2):392-9.

42. Parr BM, Noakes TD, Derman EW. Peripheral arterial disease and intermittent claudication: efficacy of short-term upper body strength training, dynamic exercise training, and advice to exercise at home. South African Medical Journal 2009;99(11):800-4.

43. Saxton JM, Zwierska I, Hopkinson K, Espigares E, Choksy S, Nawaz S, et al.Effect of upperand lower-limb exercise training on circulating soluble adhesion molecules, hs-CRP and stress proteins in patients with intermittent claudication. European Journal of Vascular and Endovascular Surgery 2008;35(5):607-13.

44. Saxton JM, Zwierska I, Blagojevic M, Choksy SA, Nawaz S, Pockley AG. Upper- versus lowerlimb aerobic exercise training on health-related quality of life in patients with symptomatic peripheral arterial disease. Journal of Vascular Surgery $2011 ; 53(5): 1265-73$.
45. Tebbutt N, Robinson L, Todhunter J, Jonker L. A plantar flexion device exercise programme for patients with peripheral arterial disease: a randomised prospective feasibility study. Physiotherapy 2011 ;97(3):244-9.

46. Walker RD, Nawaz S, Wilkinson $\mathrm{CH}$, Saxton JM Pockley AG, Wood RF. Influence of upper- and lower-limb exercise training on cardiovascular function and walking distances in patients with intermittent claudication. Journal of Vascular Surgery 2000;31(4):662-9.

47. Wang $E$, Hoff J, Loe $H$, Kaehler N, Helgerud J. Plantar flexion: an effective training for peripheral arterial disease. European Journal of Applied Physiology 2008;104(4):749-56.

48. Zwierska I, Walker RD, Choksy SA, Male JS, Pockley AG, Saxton JM. Upper- vs lower-limb aerobic exercise rehabilitation in patients with symptomatic peripheral arterial disease: a randomized controlled trial. Journal of Vascular Surgery 2005;42(6): 1 122-30.

49. Treat-Jacobson D, Henly SJ, Bronas UG, Leon AS, Henly GA. The pain trajectory during treadmill testing in peripheral artery disease. Nursing Research 2011 ;60(3 Suppl):S38-49.

50. Jones PP, Skinner JS, Smith LK, John FM, Bryant CX. Functional improvements following Stairmaster vs. treadmill exercise training for patients with intermittent claudication. Journal of Cardiopulmonary Rehabilitation 1996; 16(1):47-55.

51. Collins EG, O'Connell S, McBurney C, Jelinek C, Butler J, Reda D, et al.Comparison of walking with poles and traditional walking for peripheral arterial disease rehabilitation. Journal of Cardiopulmonary Rehabilitation and Prevention 2012;32(4):210-8. 


\section{CHAPTER 5}

\section{PHYSICAL ACTIVITY MONITORING IN PATIENTS WITH INTERMITTENT CLAUDICATION}

G.J. Lauret

H.J.P. Fokkenrood

B.L. Bendermacher M.R.M. Scheltinga J.A.W. Teijink 


\section{ABSTRACT}

\section{Objectives}

Reduced physical activity (PA) is associated with a higher mortality rate and more rapid functional decline in patients with intermittent claudication (IC). The newest generation of accelerometers can assess both direction and intensity of activities threedimensionally and may also adequately calculate energy expenditure in daily life. The aim of this study was to quantify daily PA level and energy expenditure of newly diagnosed patients with IC and healthy controls. PA outcomes are compared with contemporary public health physical activity guidelines.

\section{Methods}

Before initiating treatment, 94 patients with newly diagnosed IC and 36 healthy controls were instructed to wear a tri-axial seismic accelerometer for 1 week. Daily PA levels (in metabolic equivalents, METs) were compared with the ACSM/AHA public health PA minimum recommendations ( $\geq 64 \mathrm{METs}$. min.day, in bouts of $\geq 10$ minutes). A subgroup analysis assessed the effect of functional impairment on daily PA levels.

\section{Results}

Data from 56 IC patients and 27 healthy controls were available for analysis. Patients with IC demonstrated significantly lower mean daily PA levels $( \pm S D)$ than controls (387 $\pm 198 \mathrm{METs} \cdot \mathrm{min}$ vs. $500 \pm 156 \mathrm{MET} \cdot \mathrm{min}, \mathrm{p}=0.02$ ). This difference was solely attributable to a subgroup of IC patients with the largest functional impairment (WIQscore $<0.4)$. Only $45 \%$ of IC patients met the public health physical activity guidelines compared with $74 \%$ of the healthy controls $(p=0.01)$.

\section{Conclusions}

More than half of patients with IC do not meet recommended standards of PA. Considering the serious health risks associated with low PA levels, these findings underscore the need for more awareness to improve physical exercise in patients with IC. 


\section{INTRODUCTION}

Intermittent claudication (IC) is closely associated with cardiovascular and cerebrovascular disease..$^{1-3}$ Compared with healthy adults, IC patients are burdened with a low health-related quality of life and functional impairment during daily activities. ${ }^{4,5} \mathrm{~A}$ reduced exercise capacity and lower daily free-living physical activity (PA) level are both strong predictors of long-term mortality. In contrast, higher PA levels are associated with less functional decline..$^{6-9}$

The ACSM/AHA have issued recommendations on types and amounts of PA needed for (older) adults to improve and maintain health. ${ }^{10}$ Specific recommendations are provided that apply to adults $>65$ years of age or to adults aged 50-64 years with chronic conditions or physical functional limitations. ${ }^{11}$ All adults are advised to engage in moderate-intensity aerobic exercise for a minimum of 30 minutes on 5 days a week, or vigorous-intensity aerobic PA for a minimum of 20 minutes on 3 days a week. Activities should be performed in bouts of at least 10 minutes. Combinations of moderate- and vigorous-intensity aerobic PA can also be performed to meet these recommendations.

Metabolic equivalents (METs) are used by the ACSM/AHA as a means to express the energy expenditure or energy costs of physical activities. The total amount of PA is a function of its intensity, duration, and frequency. Accordingly, vigorous intensity activities (>6.0 METs) performed for a particular duration and frequency generate greater energy expenditure than moderate-intensity activities (3.0-6.0 METs) of the same duration and frequency. When combining the recommendations on moderateand vigorous-intensity physical activity, the daily minimum goal of PA should be in the range of 64-107 METs.min. ${ }^{10,11}$

In the literature, PA is defined and determined in different ways. Frequently used definitions of PA are "time spent in different activities (sedentary/ambulatory), number of steps, duration of walking events or the score on a specific exercise test

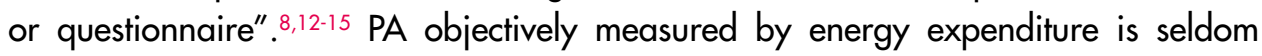
performed in patients with IC. ${ }^{15-17}$ Regularly described methods for PA measurement are PA questionnaires, pedometers, or activity monitors. However, only the last method is found capable of adequately assessing energy expenditure. ${ }^{18,19}$ The newest generation of activity monitors is based on tri-axial accelerometer techniques measuring accelerations in three dimensions that can be converted to intensities and METs. In contrast to unilateral accelerometers (or vertical accelerometers), tri-axial accelerometers also measure activities that do not include vertical movement. As such, 
PA is determined more precisely as demonstrated with high correlations between indirect calorimetry and generated MET output at different walking speeds. ${ }^{18,20,21}$ To our knowledge, no studies are available on the use of a tri-axial accelerometer in patients with IC compared with healthy adults.

The purpose of this prospective observational study was to objectively determine the PA using a tri-axial accelerometer in patients with IC and healthy adults. Furthermore, the number and percentage of participants meeting the lower limit of the ACSM/AHA recommendations for PA and public health are determined. It was hypothesised that healthy adults had a higher PA level and complied more frequently with these minimum recommendations than IC patients.

\section{MATERIALS \& METHODS}

\section{Participant selection}

Patients with clinical manifestations of IC were recruited at the outpatient clinic of Vascular Surgery of the Catharina Hospital, Eindhoven and Maxima Medical Center, Veldhoven/Eindhoven, The Netherlands. Healthy individuals recruited from family or friends of healthcare workers served as controls. All subjects gave written informed consent and all procedures described in this study were approved by the Medical Ethical Committee, Catharina Hospital, Eindhoven.

\section{Eligibility assessment}

\section{IC group}

Patients without any previous history of peripheral arterial occlusive disease (PAOD) presenting with new onset clinical manifestations of IC underwent an ankle-brachial index (ABI) measurement using standard equipment. Patients with values below 0.90 at rest or a drop in value of more than 0.15 after a standard treadmill test were considered eligible. Exclusion criteria included serious cardiopulmonarylimitations (NYHA class 3-4), critical limb ischemia or previous lower-limb amputation, use of walking aids, psychiatric instability or other serious comorbidity which possibly limited the patient's walking ability.

\section{Control group}

Healthy volunteers aged $>45$ years without a history of PAOD, cardiac or pulmonary disease were included if they were able to walk without any limitations or walking aids. Participants were excluded if an $A B I$ measurement was below 0.90 at rest or decreased more than 0.15 after a standard treadmill test. 


\section{Functional impairment and health-related quality of life scores}

After consenting to the study specifics, a Dutch validated version of the Walking Impairment Questionnaire (WIQ), 12-item Short Form Physical Functioning Summery (SF-12 PCS) and 12-item Short Form Mental Functioning Summery (SF-12 MCS) were assessed prior to treatment in the IC patients and in controls. ${ }^{22,23}$ Evidence based conservative treatment was subsequently started in the IC group. Each patient received cardiovascular risk management including antiplatelet therapy and a statin.

\section{Physical activity monitoring \\ Dynaport tri-axial seismic accelerometer}

Both groups of study participants were asked to wear a tri-axial seismic accelerometer (Dynaport MoveMonitor, McRoberts B.V., The Hague, The Netherlands) during a 1 week time period. The Dynaport MoveMonitor (size $84 \mathrm{~mm} \times 50 \mathrm{~m} \times 8 \mathrm{~mm}$; weight $55 \mathrm{~g}$ ) contains three orthogonal piezo-capacitive acceleration sensors, each measuring at a sample rate of $100 \mathrm{~Hz}$. The accelerometer has a direct current response to the Earth's gravitational field, and used a seismic or a proof mass suspended by a spring structure in a case. The case has a micro USB connection and a rechargeable battery and stores the raw data on a Micro-SD card. The Dynaport MoveMonitor is placed in a belt that is strapped around the waist. It is positioned at the base of the lumbar column just cranial to the buttocks. The Dynaport technology is validated for counting steps, detecting time spent in different activities, activity-related energy expenditure and assessment of reproducibility. 18,21,24-28

\section{Monitoring protocol}

All participants were instructed to correctly wear the Dynaport MoveMonitor for 7 consecutive days. As the device is not waterproof, participants were asked to take off the device when taking a shower or a bath. Furthermore, to achieve better compliance, participants were instructed not to wear the device during sleeping.

They were instructed to perform their regular daily activities during this week. Supervised exercise therapy (SET) was initiated after this one week monitoring period in IC patients. SET was provided by a specialised physical therapist in a community-based setting. According to the quality standards and guidelines of the Dutch ClaudicatioNet Network and Royal Dutch Society for Physical Therapy, the physical therapist assessed the pain-free walking distance (PFWD) and maximum walking distance (MWD) at baseline according to a standardised graded treadmill protocol. 29,30 


\section{Outcomes}

\section{Primary outcomes}

- The daily PA level (measured in METs.min)

- The daily PA level performed in bouts of $>10$ minutes moderate- or vigorousintensity PA (measured in METs.min)

- The number and percentage of participants meeting the lower limit of the ACSM recommendation for PA and public health (67 METs.min a day)

\section{Secondary outcomes}

- The average daily time spent in different activities (Lying, Standing, Sitting, Walking, Shuffling) and the time spent not wearing the device

- The average daily number and highest number of steps walked in one period (top period of steps)

- The average daily time spent in light-, moderate- or vigorous-intensity activity. Sedentary activity is activity with an intensity of $<1.5 \mathrm{METs}$, light activity is activity with an intensity of 1.5-3 METs, moderate activity is activity with an intensity of 3-6 METs and vigorous activity is activity with an intensity of 6-9 METs.

\section{Data Analysis}

Daily checks were made to assure proper use of the device. By definition, the device was worn correctly if it detected at least 20 steps and if it was 'worn' $\geq 10$ hours and 'not worn' for $\geq 5$ hours a day. Participants with less than 5 days of correctly wearing the device were arbitrarily excluded from analysis. Daily average outcomes were determined for each participant depending on the number of 'correctly worn days'.

\section{Subgroup analysis}

The IC group was divided into two subgroups according to the WIQ score $(<0.4$ or $\geq 0.4)$ in order to evaluate the hypothesis that IC patients with more functional impairment have a lower daily PA level. The WIQ-score was used because it takes both the patient's effort to walk and his perceived impairment into account, while estimated or treadmill walking distances do not reflect walking distances in daily life. ${ }^{31} \mathrm{~A}$ lower WIQ-score reflects higher functional impairment. A cut-off value of 0.4 was arbitrarily chosen to establish even groups. 


\section{Statistical analyses}

All analyses were performed using IBM SPSS Statistics Version 21 (SPSS Inc., Chicago, IL, USA). Categorical variables are presented as frequencies with percentages. Nominal variables are depicted as the mean \pm standard deviation in case of a normal distribution. Statistical differences between the groups were calculated with a Pearson Chi-Square test for categorical variables, an Independent sample T-test for continuous variables between two groups and a One-Way between-groups ANOVA for continuous variables between more than two groups. In case of significant differences between more than two groups, post-hoc Tukey tests were performed to assess a possible significant difference between groups. A p-value $<0.05$ was used for statistical significance.

Univariable and multivariable linear regression analysis were used to evaluate which pre-specified variables predicted daily level of physical activity. In the univariable regression analysis, each variable is included in a separate regression analysis. In the multivariable regression analysis, all statistically significant variables, identified by the univariable regression analysis, are included in one regression analysis, using the enter method. Included pre-specified variables were age, $B M I$, sex, resting $A B I, I C$ symptoms of both legs, cardiac history, pulmonary history, WIQ score, SF-12 PCS score, SF-12 MCS score, and maximal walking distance measured at baseline. Multivariable linear regression analysis using the backward elimination method was used to identify the variables included in the final regression equation. The backward method starts with all the variables in the equation and in every step variables that do not have a statistically significant influence are excluded. The criterion for removal from the regression model was a p-value of more than 0.10 . The quality of the regression models was expressed with the $\mathrm{R}^{2}$ (explained variance) for linear regression analysis.

\section{RESULTS}

\section{Group baseline characteristics}

A total of 130 participants were considered eligible (IC, $n=94$; controls, $n=36$ ). Analysis of the accelerometer data demonstrated that 56 IC patients and 27 controls met all criteria of a correctly worn device. A significant group difference regarding age, number of correctly worn days, resting ABI, WIQ-score, SF-12 PCS score and MWD was found between both groups. (Table 1) 


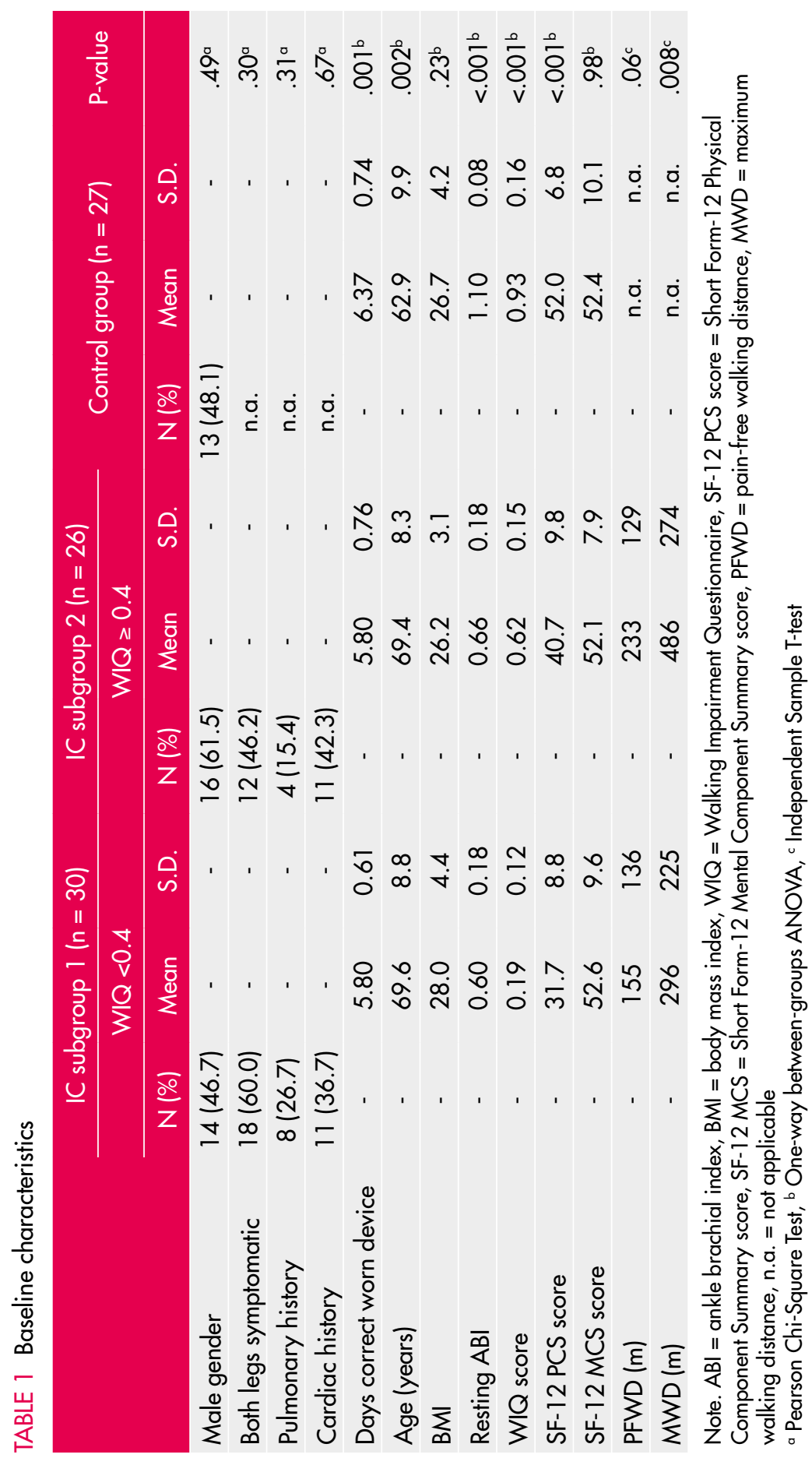




\section{Time spent in different activities or intensity-levels}

IC patients spent almost 8.5 hours a day sitting (508 \pm 108 minutes), more than 2.5 hours a day standing (165 \pm 60 minutes), more than 2 hours a day lying (132 \pm 101 minutes), 1 hour a day walking ( $65 \pm 33$ minutes) and $21 \pm 12$ minutes a day shuffling. (Table 2) Compared to the control group a significant difference was found in the time spent sitting and 'device not worn'. A significant group difference was also found regarding vigorous-intensity activities (IC: $4 \pm 8$ vs. Controls: $11 \pm 10$ minutes a day, $\mathrm{p}=0.002$ ).

\section{Step analysis}

Daily number of steps in the IC subgroups was significantly fewer compared to the control group (IC subgroup 1: $4663 \pm 3104$ vs. IC subgroup 2: $5761 \pm 2371$ vs. Controls: $6682 \pm 2701, p=0.03$ ). The highest number of steps walked in one period (top period of steps) was also significantly lower in IC patients (IC subgroup 1: $1601 \pm$ 1414 vs. IC subgroup 2: $2546 \pm 1894$ vs. Controls: $4584 \pm 4680, p<0.001$ ).

TABLE 2 Average duration of different daily activities and intensity levels

\begin{tabular}{|c|c|c|c|c|c|}
\hline & \multicolumn{2}{|c|}{ IC group $(n=56)$} & \multicolumn{2}{|c|}{ Control group $(n=27)$} & \multirow{2}{*}{ P-value ${ }^{a}$} \\
\hline & Mean & S.D. & Mean & S.D. & \\
\hline Lying (hours:min) & $2: 12$ & 101 & $2: 10$ & 62 & 0.92 \\
\hline Sitting (hours:min) & $8: 28$ & 108 & $9: 20$ & 80 & 0.03 \\
\hline Standing (hours:min) & $2: 45$ & 60 & $2: 33$ & 47 & 0.34 \\
\hline Walking (hours:min) & $1: 05$ & 33 & $1: 16$ & 29 & 0.14 \\
\hline Shuffling (hours:min) & $0: 21$ & 12 & $0: 26$ & 12 & 0.10 \\
\hline Not Worn (hours:min) & $9: 13$ & 50 & $8: 16$ & 47 & $<0.001$ \\
\hline Light activity (hours:min) & $1: 18$ & 33 & $1: 33$ & 55 & 0.13 \\
\hline Moderate activity (hours:min) & $1: 23$ & 37 & $1: 35$ & 29 & 0.11 \\
\hline Vigorous activity (hours:min) & $0: 04$ & 8 & $0: 11$ & 10 & 0.002 \\
\hline
\end{tabular}

\section{Daily physical activity level}

A statistically significant difference $(p=0.02)$ in both daily PA level and daily PA in bouts of $>10$ minutes of moderate- or vigorous intensity exercise, was found between the IC subgroups and the control group. (Figure 1,2) Post-hoc analyses showed that this difference was solely due to a significant difference between IC subgroup 1 (WIQ 
$<0.4$ ) and the control group ( $p=0.018$ ). In contrast, no significant difference in daily PA level was found between IC subgroup $2(\mathrm{WIQ} \geq 0.4)$ and the control group $(p=0.28)$.

FIGURE 1. Daily physical activity level (mean \pm S.D.)

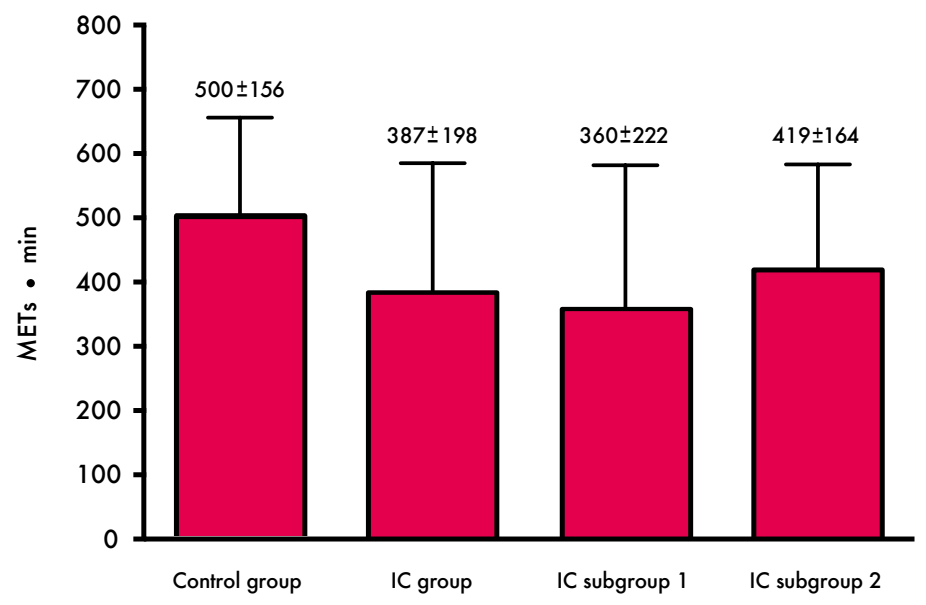

FIGURE 2. Daily physical activity level in bouts $>10$ min of moderate or vigorous activity (median, I.Q.R.)

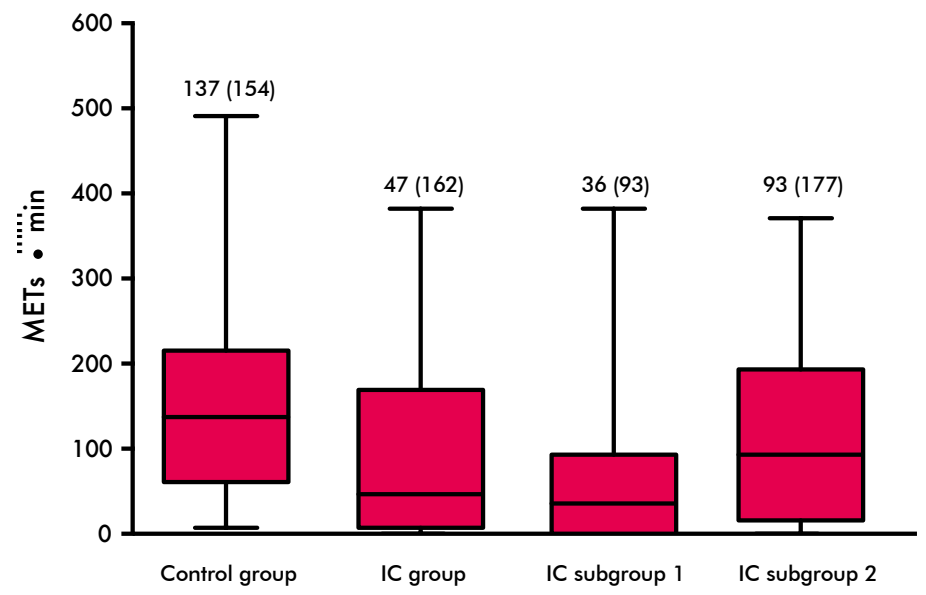


Analysis of the number of participants achieving the lower (67 METs.min a day) and upper (107 METs-min a day) limit of the ACSM minimum recommendations showed a significant difference (lower limit: $p=0.01$, higher limit: $p=0.02$ ) between the IC subgroups and the control group in favour of the healthy controls. (Figure 3 )

FIGURE 3. Adherence to ACSM recommendations for physical activity and public health

$\square$ Achieved lower limit of ACSM recommendations (67 METsmin a day)

Achieved upper limit of ACSM recommendations (107 METsmin a day)

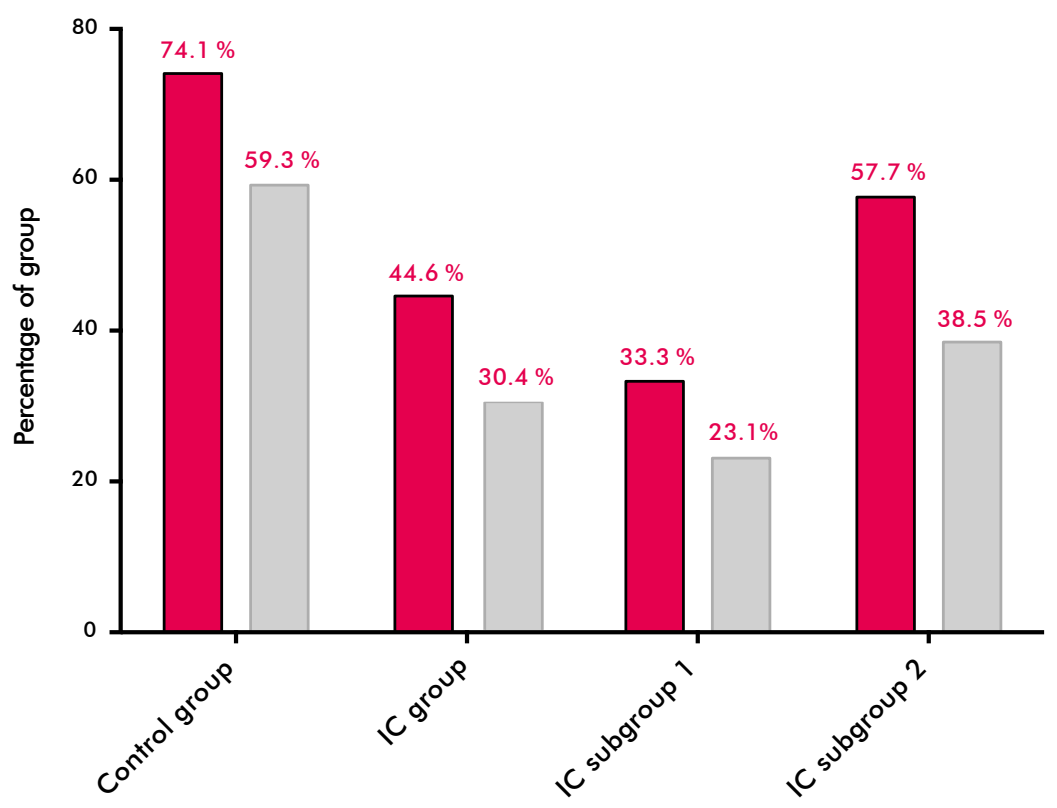

Prediction model for daily physical activity level

Univariable regression analysis revealed that age, a previous history of pulmonary disease, MWD and the WIQ, SF-12 MCS and SF-12 PCS score, all independently influenced the daily PA level. (Table 3) Multivariable regression analysis including five variables (age, pulmonary history, SF-12 PFS, SF-12 MFS, MWD) explained 53\% $(p<0.001)$ of the variance in daily PA level:

Daily PA level of IC patients (METs $\cdot \mathrm{min})=369.23-7.65 \times$ Age $+4.73 \times$ SF-12 PCS score $+6.53 \times$ SF-12 MCS score $+0.18 \times$ MWD (- 138.65 if pulmonary history is present) 


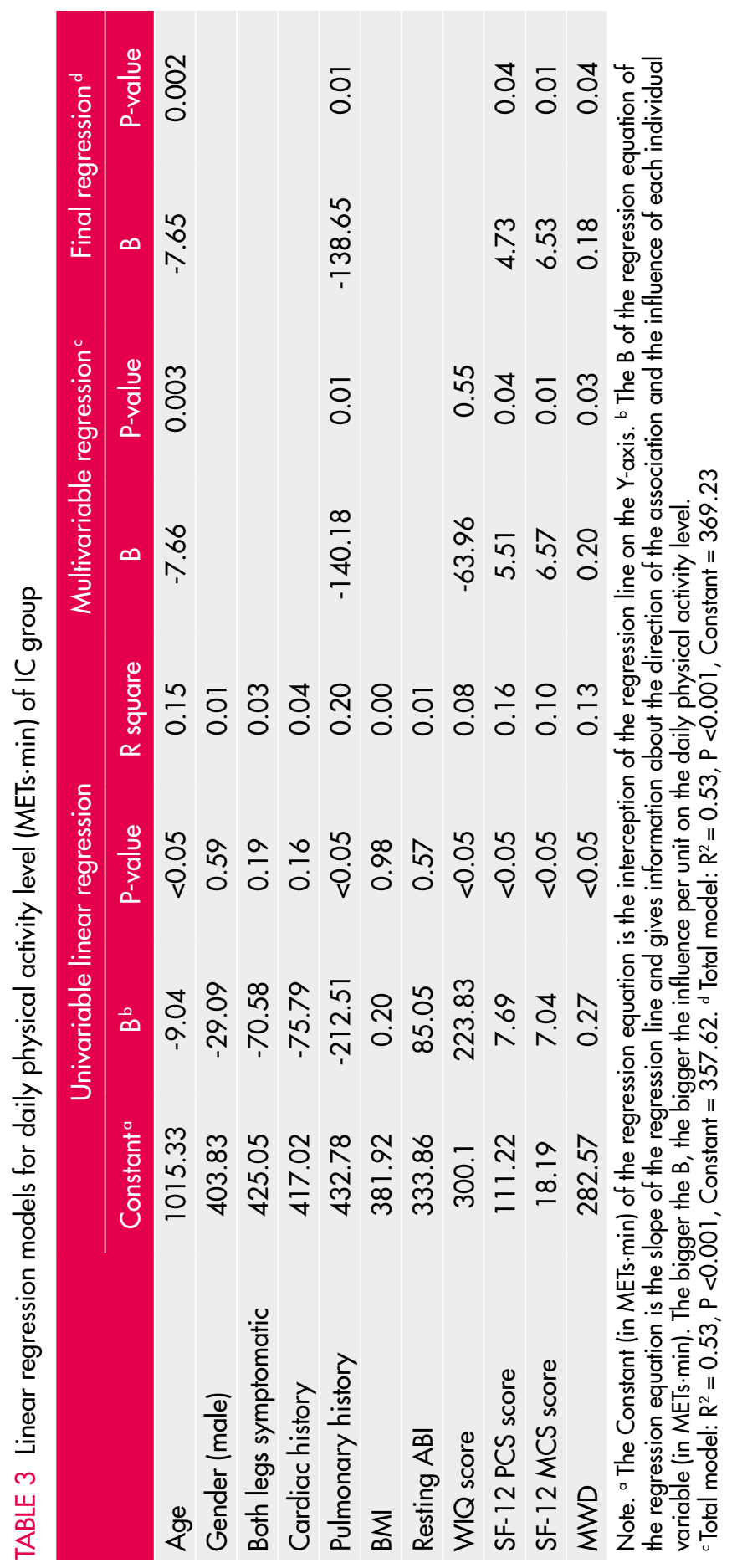




\section{DISCUSSION}

This study hypothesized that IC patients would demonstrate a lower PA level and would comply less frequently to the lower limit of the ACSM/AHA recommendations for PA and public health compared to healthy adults. In a two-day assessment of PA using a vertical accelerometer and a pedometer, one other study found that daily PA level of IC patients was $42 \%-45 \%$ lower compared to healthy subjects of similar age. ${ }^{17}$ Also using a vertical accelerometer, a second study demonstrated that patients with peripheral arterial occlusive disease (PAOD) had markedly lower PA levels compared to nonPAOD participants $(803 \pm 364 \mathrm{kcal} / \mathrm{wk}$ vs. $1750 \pm 1296 \pm \mathrm{kcal} / \mathrm{wk}) .{ }^{15}$ Ever since the ACSM/AHA issued minimum recommendations on PA and public health were issued, studies describing to what extent IC patients meet these criteria in relation to healthy adults were not performed.

The present study using newest generation of tri-axial accelerometers found that fewer than half of IC patients meet the ACSM/AHA minimum recommendations while more than one-quarter of older healthy adults do not meet these criteria.

Moreover, the daily level of PA of IC patients $(387 \pm 198)$ is much lower compared to healthy adults $(500 \pm 156)$. IC patients reporting substantial functional impairment (WIQ score $<0.4$ ) had the lowest mean daily PA level of all groups. Interestingly, the PA group difference was solely attributed to this subgroup. Only minor functional impairment in IC patients apparently does not result in lower daily PA level.

A tri-axial accelerometer allows for separately identifying various activities such as sitting, standing, lying or walking. The present study found significant group differences regarding time of sitting and 'not wearing the device'. Healthy adults appear to sit approximately one hour longer and might sleep less compared to IC patients. In contrast, walking time did not differ among groups, although the daily number of steps and highest number of steps were significantly lower in the IC group. This difference is possibly explained by a slower walk pace or cadence associated with IC. A previous study also suggested that patients with IC take fewer steps and have a lower daily average cadence compared to healthy adults. ${ }^{32}$

\section{Variables influencing daily PA level in IC patients were hitherto not identified.}

The present study revealed that age, previous history of pulmonary disease, MWD and the WIQ, SF-12 MCS and SF-12 PCS score all independently influence these levels. Pulmonary history appeared the strongest factor. However, patient with serious pulmonary conditions (affecting the patient's walking ability) were excluded from this 
study. Therefore, the effect of pulmonary history on the daily PA level in an IC patient is probably underestimated. Patients with COPD are markedly inactive in daily life compared to healthy adults. ${ }^{33}$ Therefore, physicians need to realize that patients with IC and COPD are much less active compared to IC patients without pulmonary history or healthy adults.

A low daily PA level is generally considered a strong predictor of mortality and functional decline in patients with IC. ${ }^{6-9}$ More than light-intensity weekly PA improves 5-year survival rate significantly compared to light-intensity or no physical activity. ${ }^{7}$ For this reason, one could argue that the PA level is a valid outcome parameter in evaluating treatment effects of any intervention for IC. Current outcome parameters including maximum- or pain-free walking distances only describe the patient's exercise capacity while it does not reflect to what extent this capacity is used. In contrast, daily PA levels, provide objective information on the frequency, duration and intensity of exercise. Combined with a functional impairment score such as the WIQ-score, physicians may objectively monitor the patient's treatment progress.

Future studies should focus on the effects of different treatment strategies on PA.

A rehabilitation program such as supervised exercise therapy (SET) improves walking performance and functional impairment. Most programmes promote, besides supervised sessions, daily home-based exercise in order to increase the patient's daily PA. No studies have substantiated the effect of SET on the daily level of PA yet. Therefore, accelerometer studies before and after different treatment strategies may shed light on these issues.

\section{Study limitations}

The present study is limited by the number of excluded participants. In total, 38 participants in the IC group (40.4\%) and nine controls $(25.0 \%)$ were excluded because the device was not correctly worn for $\geq 5$ days. By definition, a correctly worn day was considered a day that the device was worn for $\geq 10$ hours, not worn for $\geq 5$ hours, and with at least 20 steps. These strict criteria were a priori determined to prevent bias. For instance, if a participant wore the device for only 5 hours one day, unrepresentative data on the overall person's PA would have been collected. The high dropout rate may have potentially biased the PA results, most likely by overestimating the current PA level in IC patients. Lack of motivation or misunderstanding the monitoring protocol could have led to incorrect use. A substantial number of participants said they had forgotten the end date of the monitoring period. In a few instances, participants fell asleep with the device still strapped to their waist. In our monitoring protocol, participants were asked 
to remove the device before sleeping to improve compliance. If the device was worn during the night, that day was considered by definition "not correctly worn". We are not sure if compliance could have been higher if participants wore the device constantly. In future studies, regular checkups by phone calls could be employed as a means of increasing compliance and checking the understanding of the monitoring protocol.

For clinical practice one could consider a shorter monitoring period, providing results are summarised in an average daily PA level. However, the accuracy of PA results from a shorter monitoring period should first be validated since these could vary. Only a few measurement failures with the tri-axial accelerometer were experienced. The Dynaport Move-Monitor may fail if the battery is not fully charged or if the device is worn backwards. The device indicates if it is fully charged, minimising the chance of human errors. However, in a handful of cases the device did not measure for 7 days because the investigators replaced the original SD cards with new ones. The new SD cards appeared to consume more battery energy than the original ones. Therefore the device could only measure for 5 or 6 days. Besides this error, we did not experience device malfunction. The investigators explicitly explained how to wear the device to minimise the chance that participants might wear the device backwards. This could be explained quite easily since the device is worn correctly if the word 'McRoberts' on the outside of the device could be read. Therefore this error did not occur during any of the worn days.

Another study limitation is the lack of an age-matched control group. Although groups were initially matched for age and sex, a significant difference in age was present between the IC and control group once the IC group drop out rate appeared high. However, a sensitivity analysis including healthy adults aged $\leq 60$ years $(n=14$ ) revealed no significant difference in daily PA level in comparison to 13 other healthy adults aged $>60$ years $(526 \pm 160$ METs $\cdot \min$ vs. $471 \pm 151$ METs $\cdot \min , p=0.37)$ although these groups may have been underpowered.

\section{CONCLUSIONS}

More than half of patients with IC do not meet the minimum recommendations for PA and public health. Compared with healthy adults, they have a significantly lower daily PA level. PA differences are due to an IC subgroup with severe functional impairments. Because a low PA level is considered a strong predictor of mortality and functional decline, all health-care workers should actively encourage physical activities in IC patients. Future studies should assess the effects of treatment strategies including supervised exercise therapy on daily PA levels. 


\section{REFERENCES}

1. Norgren L, Hiatt WR, Dormandy JA, Nehler MR, Harris KA, Fowkes FG. Inter-Society Consensus for the Management of Peripheral Arterial Disease (TASC II). J Vasc Surg. 2007;45 Suppl S:S5-67.

2. Criqui MH. Systemic atherosclerosis risk and the mandate for intervention in atherosclerotic peripheral arterial disease. Am J Cardiol. $2001 ; 88(7 B): 43 J-7 J$.

3. Criqui MHL, R. D.; Fronek, A. Mortality over a period of 10 years in patients with peripheral arterial disease. N Engl J Med. 1992;326:7.

4. Regensteiner JG, Hiatt WR, Coll JR, Criqui MH, Treat-Jacobson D, McDermott MM, et al. The impact of peripheral arterial disease on healthrelated quality of life in the Peripheral Arterial Disease Awareness, Risk, and Treatment: New Resources for Survival (PARTNERS) Program. Vasc Med. 2008;13(1):15-24.

5. Dumville JC, Lee AJ, Smith FB, Fowkes FG. The health-related quality of life of people with peripheral arterial disease in the community: the Edinburgh Artery Study.Br J Gen Pract. 2004;54(508):826-31.

6. Leeper NJ, Myers J, Zhou M, Nead KT, Syed A, Kojima $Y$, et al. Exercise capacity is the strongest predictor of mortality in patients with peripheral arterial disease. J Vasc Surg. 2013;57(3):72833.

7. Gardner AW, Montgomery PS, Parker DE. Physical activity is a predictor of all cause mortality in patients with intermittent claudication. J Vasc Surg. 2008;47(1):117-22.

8. Garg PK, Liu K, Tian L, Guralnik JM, Ferrucci L, Criqui $M H$, et al. Physical activity during daily life and functional decline in peripheral arterial disease. Circulation. 2009;1 19(2):251-60.

9. McDermott MM, Liu K, Ferrucci L, Criqui $M H$, Greenland P, Guralnik JM, et al. Physical performance in peripheral arterial disease: a slower rate of decline in patients who walk more. Ann Intern Med. 2006;144(1):10-20.

10. Haskell WL, Lee IM, Pate RR, Powell KE, Blair $\mathrm{SN}$, Franklin BA, et al. Physical activity and public health: updated recommendation for adults from the American College of Sports Medicine and the American Heart Association. Circulation. 2007;1 16(9):1081-93.
11. Nelson ME, Rejeski WJ, Blair SN, Duncan PW Judge JO, King $A C$, et al. Physical activity and public health in older adults: recommendation from the American College of Sports Medicine and the American Heart Association. Circulation. 2007;1 16(9):1094-105.

12. Clarke CL, Holdsworth RJ, Ryan CG, Granat $M H$. Free-living physical activity as a novel outcome measure in patients with intermittent claudication. Eur J Vasc Endovasc Surg. 2013;45(2):162-7.

13. Gardner AW, Montgomery PS, Scott KJ, Blevins SM, Afaq A, Nael R. Association between daily ambulatory activity patterns and exercise performance in patients with intermittent claudication. J Vasc Surg. 2008;48(5):123844.

14. McDermottMM, Liu K, Ferrucci L, Tian L, Guralnik $J M$, Liao $Y$, et al. Greater sedentary hours and slower walking speed outside the home predict faster declines in functioning and adverse calf muscle changes in peripheral arterial disease. $J$ Am Coll Cardiol. 2011;57(23):2356-64.

15. McDermott MM, Liu K, O'Brien E, Guralnik $J M$, Criqui MH, Martin GJ, et al. Measuring physical activity in peripheral arterial disease: a comparison of two physical activity questionnaires with an accelerometer. Angiology. 2000;51(2):91-100.

16. Sieminski DJ, Cowell LL, Montgomery PS, Pillai SB, Gardner AW. Physical activity monitoring in patients with peripheral arterial occlusive disease. J Cardiopulm Rehabil. 1997;17(1):437.

17. Sieminski DJ, Gardner AW. The relationship between free-living daily physical activity and the severity of peripheral arterial occlusive disease. Vasc Med.1997;2(4):286-91.

18. van Hees VT, van Lummel RC, Westerterp KR. Estimating activity-related energy expenditure under sedentary conditions using a tri-axial seismic accelerometer. Obesity(Silver Spring). 2009; 17(6):1287-92.

19. Gardner AW, Poehlman ET. Assessment of freeliving daily physical activity in older claudicants: validation against the doubly labeled water technique. J Gerontol A Biol Sci Med Sci. 1998;53(4):M275-80. 
20. Van Remoortel H, Giavedoni S, Raste Y, Burtin C, Louvaris Z, Gimeno-Santos E, et al. Validity of activity monitors in health and chronic disease: a systematic review. Int J Behav Nutr Phys Act. 2012;9:84.

21. Van Hees VT, Slootmaker SM, De Groot G, Van Mechelen W, Van Lummel RC. Reproducibility of a triaxial seismic accelerometer (DynaPort). Med Sci Sports Exerc. 2009;41(4):810-7.

22. Verspaget $M$, Nicolai $S P$, Kruidenier $L M$, Welten RJ, Prins MH, Teijink JA. Validation of the Dutch version of the Walking Impairment Questionnaire. Eur J Vasc Endovasc Surg 2009;37(1):56-61.

23. Gandek B, Ware JE, Aaronson NK, Apolone G, Biorner JB, Brazier JE, et al. Crossvalidation of item selection and scoring for the SF-12 Health Survey in nine countries: results from the IQOLA Project. International Quality of Life Assessment. J Clin Epidemiol. 1998;51(11):1171-8.

24. Gardner AW, Skinner JS, Cantwell BW, Smith LK. Progressive vs single-stage treadmill tests for evaluation of claudication. Med Sci Sports Exerc. 1991;23(4):402-8.

25. Jongert MWH, H.J.; van Hoek, J. [Guideline intermittent claudication from the Royal Dutch Society of Physical Therapy]. Ned Tiidschr Fysioth. 2003;6:50.

26. Langer D, Gosselink R, Sena R, Burtin C, Decramer $M$, Troosters T. Validation of two activity monitors in patients with COPD. Thorax. 2009;64(7):641-2.

27. Houdiik H, Appelman FM, Van Velzen JM, Van der Woude LH, Van Bennekom CA. Validity of DynaPort GaitMonitor for assessment of spatiotemporal parameters in amputee gait. J Rehabil Res Dev. 2008;45(9):1335-42.
28. Dijkstra B, Kamsma Y, Zijlstra W. Detection of gait and postures using a miniaturised triaxial accelerometer-based system: accuracy in community-dwelling older adults. Age Ageing. 2010;39(2):259-62.

29. Dijkstra B, Ziilstra W, Scherder E, Kamsma $Y$. Detection of walking periods and number of steps in older adults and patients with Parkinson's disease: accuracy of a pedometer and an accelerometry-based method. Age Ageing. 2008;37(4):436-41.

30. Van Remoortel $H$, Raste $Y$, Louvaris $Z$, Giavedoni S, Burtin C, Langer D, et al. Validity of six activity monitors in chronic obstructive pulmonary disease: a comparison with indirect calorimetry. PLoS One. 2012;7(6):e39198.

31. Frans FA, Zagers MB, Jens $S$, Bipat $S$, Reekers JA, Koelemay MJ. The relationship of walking distances estimated by the patient, on the corridor and on a treadmill, and the Walking Impairment Questionnaire in intermittent claudication. JVasc Surg. 2013;57(3):720-7 el.

32. Gardner AW, Montgomery PS, Scott KJ, Afaq A, Blevins SM. Patterns of ambulatory activity in subjects with and without intermittent claudication. J Vasc Surg. 2007;46(6):1208-14.

33. Pitta F, Troosters $T$, Spruit MA, Probst VS, Decramer M, Gosselink R. Characteristics of physical activities in daily life in chronic obstructive pulmonary disease. Am J Respir Crit Care Med. 2005; 171(9):972-7. 



\section{CHAPTER 6}

THE EFFECT OF SUPERVISED EXERCISE THERAPY ON PHYSICAL ACTIVITY AND AMBULATORY ACTIVITIES IN PATIENTS WITH INTERMITTENT CLAUDICATION

H.J.P. Fokkenrood

G.J. Lauret

N. Verhofstad

B.L. Bendermacher M.R.M. Scheltinga J.A.W. Teijink 


\section{ABSTRACT}

\section{Objectives}

Intermittent claudication (IC) is associated with a reduction in physical activity (PA) and a more rapid functional decline leading to a higher mortality rate compared to healthy individuals. Supervised exercise therapy (SET) is known to increase the walking capacity of IC patients. However, it is unclear whether SET increases PA. The aim of this study was to investigate the effect of SET on PA levels and ambulatory activities in patients with IC.

\section{Design \& Methods}

Newly diagnosed IC patients were requested to wear an activity monitor one week prior to and one week immediately following 3 months of SET. Primary outcome was the percentage of patients meeting the minimum recommendations of PA (ACSM) AHA public health, $\geq 67 \mathrm{METs}$. min.day, in bouts of $\geq 10 \mathrm{~min}$ ) at baseline and after 3 months of SET. Additionally, daily PA level (metabolic equivalents, METs.min), duration of ambulatory activities, daily number of steps, pain free walking distance (PFWD), maximal walking distance (MWD) and SF-36 health surveys were compared before and after SET.

\section{Results}

Data of 41 participants were available for analysis. A higher number of participants met the ACSM minimum recommendation for PA at the 3 months follow up (baseline: 43\%; 3 months: $63 \%, p=.003$ ). Despite significant increases in PFWD (baseline: $210 \mathrm{~m}$; 3 months: $390 \mathrm{~m}, \mathrm{p}=.001$ ), MWD (baseline: $373 \mathrm{~m} ; 3$ months: $555 \mathrm{~m}, \mathrm{p}=.002$ ) and physical functioning score (SF-36) following SET, no increase in mean daily PA level was found ( $395 \pm 220$ vs. $411 \pm 228$ METs.min, $\mathrm{p}=.43$ ). Furthermore, total number of steps and time spent in ambulatory activities did not change following SET.

\section{Conclusion}

Three months of SET for intermittent claudication leads to more patients meeting the ACSM/AHA public health minimum recommendations of PA. Assessment of PA may be incorporated as outcome parameter in future research comparing different treatment modalities for peripheral arterial disease. 


\section{INTRODUCTION}

Most patients with intermittent claudication (IC) demonstrate a limited exercise performance and have increased risks on cardio- and cerebrovascular events, reduced quality of life (QoL) and higher mortality rates.' Primary treatment of IC includes supervised exercise therapy (SET) as suggested by several international guidelines. ${ }^{1-3}$ SET significantly improves the maximal and pain-free walking distances and is costeffective. ${ }^{4-8}$ However, the question arises whether effectiveness of PAD treatment modalities should be evaluated on the basis of changes in walking capacity only.

Individuals who are regularly physically active report better overall health, lower health care expenditures and fewer mobility limitations than their sedentary counterparts. 9,10 The American Heart Association (AHA) and the American College of Sports Medicine (ACSM) provide specific recommendations of various types of activity to stimulate the elderly. Tailored recommendations are provided that apply to adults with clinically significant chronic conditions or functional limitations that affect mobility and activity. ${ }^{11}$

Guidelines suggest to engage in moderate-intensity aerobic exercise for a minimum of 30 minutes during five days a week, or a minimum of 20 minutes of vigorous-intensity activity on three days each week, or some combination of the two. ${ }^{11}$ Activities should be performed in bouts of at least 10 minutes. Intensity of effort is assessed in absolute terms by estimating the actual metabolic cost of a given activity and assigning intensity accordingly. ${ }^{12}$ A metabolic equivalent (MET) is an estimate of oxygen consumption at rest. A three-MET activity would be an activity that utilizes roughly three times the amount of resting energy expenditure. Accordingly, vigorous intensity activities (>6.0 METs) performed for a particular duration and frequency generate greater energy expenditure than moderate-intensity activities (3.0-6.0 METs) of the same duration and frequency. When combining the recommendations on moderate- and vigorousintensity physical activity (PA), the daily minimum goal of physical activity (PA) should be in the range of 64-107 METs.min. ${ }^{11}$

Recent studies demonstrated that free-living daily PA levels in IC patients are lower compared to healthy controls. ${ }^{13-16}$ However, PA is scantly used as an outcome parameter in trials concerning IC treatment. ${ }^{15,17-21}$ It may be relevant to study the potential beneficial effects of SET on PA level and exercise behaviour in IC patients. If PA levels improve following SET, cardiovascular risk reduction and improved quality of life may follow on the long term. ${ }^{22,23}$ Although SET has proven effective in improving walking distances 8,19, one can question whether an increased walking capacity is of benefit during the 
patient's daily life activities. In other words, when an IC patient is able to walk further, this finding does not necessarily implicate that PA levels and/or exercise behavior are positively influenced. A potential goal of future PAD treatment should perhaps be aimed at increasing physical activity.

The aim of this study is to assess the effect of a SET program on PA and walking behavior in an IC population. In addition, we hypothesize that a SET induced increased walking distance would improve PA levels in IC patients.

\section{METHODS}

The present study was executed between January 2013 and September 2013. The study was conducted following a previous study that was executed to quantify daily PA levels and energy expenditure in newly diagnosed IC patients. ${ }^{16}$

Patients were recruited from a population presenting with new onset manifestations of IC at outpatient departments of Vascular Surgery of the Catharina Hospital, Eindhoven and Maxima Medical Centre, Veldhoven/Eindhoven, The Netherlands. Following history taking and a physical examination, patients suspected of IC underwent an ankle-brachial index (ABI) measurement using standard equipment. Patients with values below 0.90 at rest or a drop in value of more than 0.15 after a standardised treadmill test (at $3.2 \mathrm{~km} / \mathrm{h}$ and a $10 \%$ incline) were considered eligible.

Exclusion criteria were serious cardiopulmonary limitations (NYHA class 3-4), critical limb ischemia or previous lower-limb amputation, use of walking aids, psychiatric instability or other serious comorbidity which possibly limited the patient's walking ability. Patients who were treated with invasive revascularizations during follow up were excluded as well.

Subjects that were willing to participate provided a written informed consent. All procedures described in this study were approved by the Medical Ethical Committee, Catharina Hospital, Eindhoven.

\section{Study protocol}

All participants received a detailed instruction on all specifics regarding proper handling and wearing of the DynaPort MoveMonitor (McRoberts BV, The Hague, the Netherlands) and were asked to wear the device correctly for 7 consecutive days. As the device is not waterproof, participants were instructed to remove the device when 
taking a shower or a bath. Participants were also asked to perform their normal daily activities during this week and not to wear the device during sleeping. A 3 months period of SET was commenced immediately after the first week monitoring period. Patients were referred to a local physical therapist, which were trained in providing SET according to the Royal Dutch Society for Physical Therapy guidelines and the Dutch ClaudicatioNet quality standards. ${ }^{24}$

The educational program ensured that all patients received SET according to the guidelines of the Royal Dutch Society for Physical Therapy. ${ }^{25}$ The physical therapist assessed a baseline and a 3 months pain-free walking distance (PFWD) and maximum walking distance (MWD) using a standardised graded treadmill protocol (walking at 3.2 kilometer per hour $(\mathrm{km} / \mathrm{h})$ with a $0 \%$ incline that increases by $2 \%$ every 2 minutes till a maximum of $10 \%$ ). ${ }^{26,27}$ Patients were tested for a maximum of 30 minutes $(1600 \mathrm{~m})$. Evidence-based cardiovascular risk management including antiplatelet and statin therapy was standardly initiated, in case patients were not taking this medication already. After 3 months, SET outcome was discussed with the patient followed by a second 7-day period of wearing the DynaPort MoveMonitor.

\section{Monitoring of physical activity and walking impairment}

The DynaPort MoveMonitor, a tri-axial seismic accelerometer, was used to measure PA. The DynaPort MoveMonitor was placed in a belt that was strapped around the waist. It was positioned at the base of the lumbar column just cranial to the buttocks. This accelerometer was chosen because of an optimal correlation between indirect calorimetry and generated MET output whereas walking speed was correctly measured in a population with chronic obstructive pulmonary disease. ${ }^{28}$ Moreover, this activity monitor was recently validated in an IC population. ${ }^{29}$ The device consists of a triorthogonal orientated piezo-capacitive acceleration sensor, a rechargeable battery and removable SD card to store the acceleration data. The raw acceleration data lend itself to a pattern recognition approach using logical algorithms (MoveMonitor analysis software, version 2.6) for the classification of postures (lying, sitting and standing) and motions (locomotion and shuffling). General and specifics of the software were provided in several previously published articles. ${ }^{16,30-31}$ A Dutch validated version of the Walking Impairment Questionnaire (WIQ) and a 36-item Short Form Health Survey version 2 (SF-36) were assessed at baseline and after the 3 months of SET treatment. ${ }^{32-34}$ 


\section{Primary outcomes}

- Percentage of participants meeting the ACSM lower limit recommendation for PA and public health (67 METs.min a day)

- Percentage of participants meeting the ACSM higher limit recommendation for PA and public health (107 METs.min a day)

\section{Secondary outcomes}

- Daily PA level (measured in METs.min)

- Daily PA level performed in bouts of >10 minutes of moderate or vigorous intensity (measured in METs.min)

- Mean time spent per day during different activities (in minutes; lying, standing, sitting, walking, shuffling, locomotion, not wearing the device)

- Mean number of steps walked in one period and maximal number per day (top period of steps)

- Activity per day (in minutes; sedentary activity: intensity <1.5 METs; light: 1.5-3 METs; moderate: 3-6 METs; vigorous: 6-9 METs)

\section{Data Analysis}

Data checks per patient for each study day were deemed necessary to assure proper use of the device. By definition, the device was 'worn correctly' during a day if it detected a minimum of 20 steps and if it was 'worn' $\geq 9$ hours and if it was 'not worn' for $\geq 5$ hours a day. Moreover, patients were arbitrarily excluded from analysis if the device was worn during less than 5 days, to filter out incorrect or unrepresentative data. Mean time that was spent per day was determined for each participant depending on the number of 'correctly worn days'. Analysis was performed on patients with both a correct baseline and follow-up measurement.

\section{Statistical analyses}

All analyses were performed using IBM SPSS Statistics Version 21 (SPSS Inc., Chicago, IL, USA). Categorical variables are presented as frequencies with percentages. A Pearson Chi-Square test was used to identify differences for categorical variables. To obtain an impression of the distribution, values were analyzed for skewness and kurtosis. Nominal normally distributed variables are depicted as the mean \pm standard deviation (SD), while non-normally distributed values are reported as median \pm interquartile range (IQR). 
Statistical differences between the included and excluded study populations were analysed with an independent Students T-test, while differences between time periods were calculated with a paired sample T-test or with Pearson correlation coefficients for continuous normal distributed variables. A Wilcoxon signed-rank test or Spearman's rho were utilized for non-normal distributed continuous variables. A p-value $<0.05$ was used for statistical significance.

\section{RESULTS}

\section{Baseline characteristics}

A total of 126 IC patients wore the device correctly at baseline and were considered eligible for this study (Figure 1). Analysis of the accelerometer data demonstrated that 17 subjects did not wore the device correctly for $\geq 5$ days at follow up. In six cases the internal memory card malfunctioned and data were not stored, another eight participants refused a follow up measurement and another 38 subjects were lost to follow up (36.5\%). As a consequence, data of 57 participants could be used for analysis $(45.2 \%)$, of which 10 patients received a vascular intervention during the study period and 6 did not receive SET due to inadequate health care insurance. The present study reflects the results obtained from 41 patients $135.3 \%$ of the total study population). Baseline characteristics regarding both the included and excluded study population are depicted in Table 1.

\section{ACSM recommendations in IC patients}

A total of 18 participants achieved the lower limit of the ACSM recommendations (67 METs.min a day) and 12 of them even met the ACSM upper limit (107 METs.min a day) as well at baseline line. The number of participants meeting the lower limit of the ACSM recommendations was significantly lower at baseline $(n=18,43 \%)$ compared to the numbers at 3 months of follow up ( $n=26,63 \%, p=.003$, Figure 2 ). Of the initial 18 participants that achieved the lower limit of the ACSM recommendations at baseline, 16 achieved the lower limit at follow up (Figure 2) again, while the remaining other two did not. From the 12 participants who achieved the higher limit of the ACSM recommendations at baseline $(29 \%)$, eight achieved the higher limit again after 3 months (Figure 2). In total 14 subjects met the ACSM higher limit (34\%). 
FIGURE 1. Study population flowchart.

$$
\begin{aligned}
& \text { Eligible patients with a correct } \\
& \text { baseline measurement } \\
& (n=126)
\end{aligned}
$$

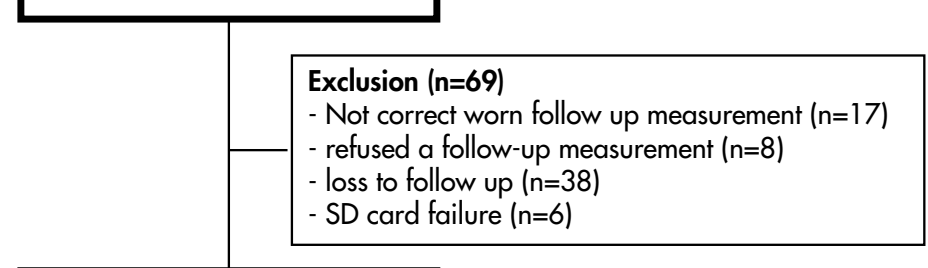

Eligible patients for analysis $(n=57)$

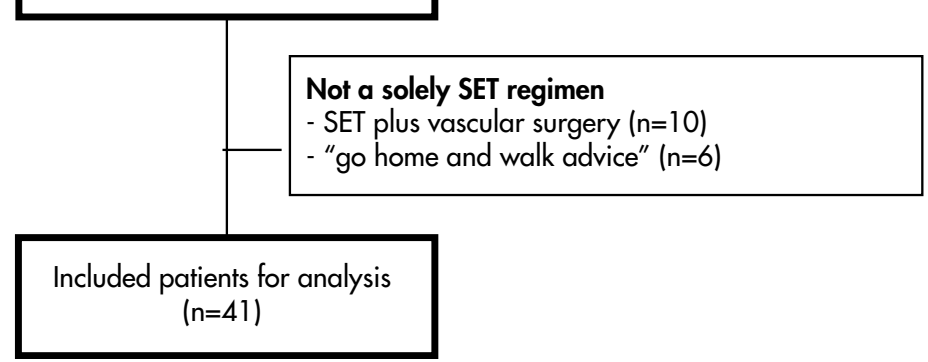

Note. Correct worn = the device was "worn correctly" during a day if it detected a minimum of 20 steps and if it was "worn" for $\geq 9$ h and "not worn" for $\geq 5$ h a day; SD = secure digital; SET = supervised exercise therapy.

FIGURE 2. Adherence to the American College of Sports Medicine's (ACSM) minimum recommended level of PA (67 METs/day) at baseline and at the 3-month follow up (FU).

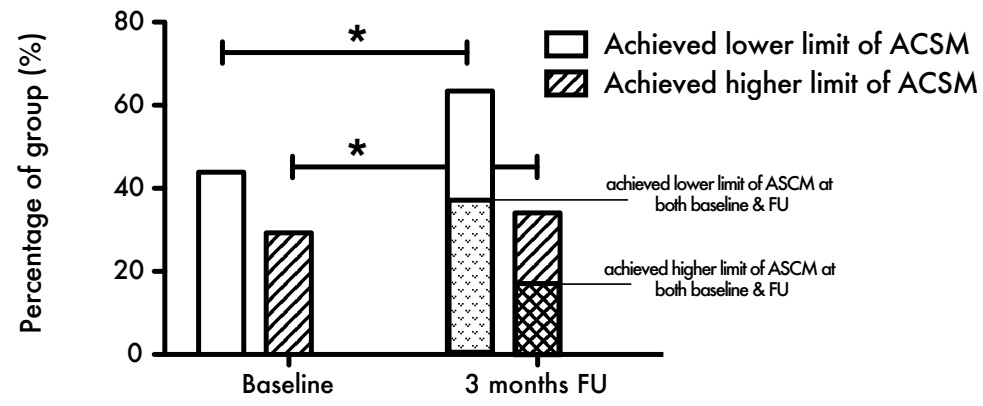

Note. ${ }^{*} \mathrm{p}<.05$ 
TABLE 1 Baseline characteristics

\begin{tabular}{|c|c|c|c|}
\hline & \multicolumn{2}{|c|}{$\begin{array}{l}\text { Total Study population } \\
(\mathrm{n}=126)\end{array}$} & \multirow[b]{2}{*}{ P-value } \\
\hline & $\begin{array}{c}\text { Included patients } \\
(\mathrm{n}=41)\end{array}$ & $\begin{array}{l}\text { Excluded patients } \\
(\mathrm{n}=85)\end{array}$ & \\
\hline Male (N - \%) & $20(48.8 \%)$ & $49(57.6 \%)$ & .45 \\
\hline Both legs symptomatic (N - \%) & 19 (46.3\%) & $38(44.7 \%)$ & .29 \\
\hline Age (years) (mean \pm SD) & $69 \pm 11$ & $68 \pm 10$ & .42 \\
\hline $\mathrm{BMI}\left(\mathrm{kg} / \mathrm{m}^{2}\right)($ mean $\pm \mathrm{SD})$ & $27.4 \pm 4.1$ & $26.7 \pm 4.0$ & .37 \\
\hline Worst $A B I$ in rest (mean $\pm S D$ ) & $0.61 \pm 0.20^{a}$ & $0.63 \pm 0.19 d$ & .50 \\
\hline Worst $A B I$ after effort (mean $\pm S D$ ) & $0.43 \pm 0.21^{b}$ & $0.44 \pm 0.22 \mathrm{e}$ & .85 \\
\hline \multicolumn{4}{|l|}{ Duplex right leg ${ }^{c}$} \\
\hline No leasions & $6(14.6 \%)$ & $\mathrm{n} / \mathrm{a}$ & \\
\hline Aortoiliac & $6(14.6 \%)$ & $\mathrm{n} / \mathrm{a}$ & \\
\hline Femoropopliteal & $12(29.3 \%)$ & $n / a$ & \\
\hline Combination & $5(12.2 \%)$ & $\mathrm{n} / \mathrm{a}$ & \\
\hline \multicolumn{4}{|l|}{ Duplex left leg c } \\
\hline No leasions & $10(24.4 \%)$ & $\mathrm{n} / \mathrm{a}$ & \\
\hline Aortoiliac & $4(9.8 \%)$ & $n / a$ & \\
\hline Femoropopliteal & $12(29.3 \%)$ & $\mathrm{n} / \mathrm{a}$ & \\
\hline Combination & $5(12.2 \%)$ & $\mathrm{n} / \mathrm{a}$ & \\
\hline Pulmonary history ( $\mathrm{N}$ - \%) & $9(22.0 \%)$ & $16(18.8 \%)$ & .81 \\
\hline Cardiac history (N - \%) & $10(24.4 \%)$ & 29 (34.1\%) & .33 \\
\hline
\end{tabular}

Note. $\mathrm{BMI}=$ body mass index; $\mathrm{ABI}=$ ankle-brachial index; $\mathrm{NA}=$ not available. ${ }^{a}$ Values based on 29 patients. ${ }^{b}$ Values based on 38 patients. ${ }^{c}$ Values based on 82 patients. ${ }^{d}$ Values based on 30 patients. ${ }^{e}$ Values based on 73 patients.

Daily level of physical activity in IC patients

Mean daily PA level (in METs.min) was normally distributed. No significant difference in mean daily PA level was found after 3 months of SET (baseline: $395 \pm 220$ METs.min vs. 3 months: $411 \pm 228 \mathrm{METs} \cdot \mathrm{min}, \mathrm{p}=.43$; Figure 3). Moreover, daily PA levels performed in bouts of $>10$ minutes were also not significantly different after 3 months $(p=.13$; Figure 3). No significant correlation coefficients were found between the change in mean daily PA (METs.min) or mean daily PA in bouts and the increase in MWD ( $\triangle$ mean daily PA vs. $\triangle M W D: r=.21, p=.19 ; \triangle$ mean daily PA in bouts vs. $\triangle M W D: r=.30, p=.06$ ). 
TABLE 2 Mean daily activities and duration at different intensity levels at baseline and at the 3-month follow up.

\begin{tabular}{|c|c|c|c|}
\hline & \multicolumn{2}{|c|}{ Total Study population ( $n=41$ ) } & \multirow[t]{2}{*}{ P-value ${ }^{a}$} \\
\hline & $\begin{array}{c}\text { Baseline } \\
\text { Median (IQR) }\end{array}$ & $\begin{array}{c}3 \text { months } \\
\text { Median (IQR) }\end{array}$ & \\
\hline Device worn correctly (days) & $7(6-7)$ & $7(6-7)$ & \\
\hline Time worn (min) & $910(880-1006)$ & $932(899-1043)$ & .06 \\
\hline Lying (min) & $139(72-270)$ & $182(99-270)$ & .27 \\
\hline Sitting (min) & $462(390-580)$ & $512(397-607)$ & .24 \\
\hline Standing (min) & $151(108-221)$ & $168(123-209)$ & .40 \\
\hline Walking (min) & $64(40-87)$ & $61(42-88)$ & .87 \\
\hline Shuffling (min) & $20(13-25)$ & $17(13-32)$ & .70 \\
\hline Locomotion (min) & $89(61-116)$ & $82(60-125)$ & .84 \\
\hline Not Worn (min) & $529(434-559)$ & $509(397-541)$ & .11 \\
\hline Steps (mean \pm SD) & 5732 (3187) & 5805 (3336) & $.43^{b}$ \\
\hline $\begin{array}{l}\text { Top period consecutive steps } \\
\text { (mean } \pm \text { SD) }\end{array}$ & $1914(1473)$ & $2390(1904)$ & $.03^{b}$ \\
\hline Light activity (min) & $66(50-91)$ & $64(51-91)$ & .29 \\
\hline Moderate activity (min) & $83(59-107)$ & $81(58-106)$ & .81 \\
\hline $\begin{array}{l}\text { Vigorous activity ( } \mathrm{min} \text { ) } \\
\text { Note. IQR = interquartile rang } \\
\text { (METs); moderate activity = a } \\
\text { intensity of 6.1-9.0 METs. a W }\end{array}$ & $\begin{array}{l}1.3(0.4-6.2) \\
\text { activity }=\text { activity } \\
\text { ith an intensity of } 3 \\
\text { signed rank test. b }\end{array}$ & $\begin{array}{l}0.9(0.4-7.9) \\
\text { tensity of } 1.5-3.0 \mathrm{n} \\
\text { ETs; vigorous activi } \\
\text { st. }\end{array}$ & $\begin{array}{l}.97 \\
\text { equivale } \\
\text { ivity with }\end{array}$ \\
\hline
\end{tabular}

FIGURE 3. Mean daily physical activity (PA) level at baseline and at 3-month follow up.

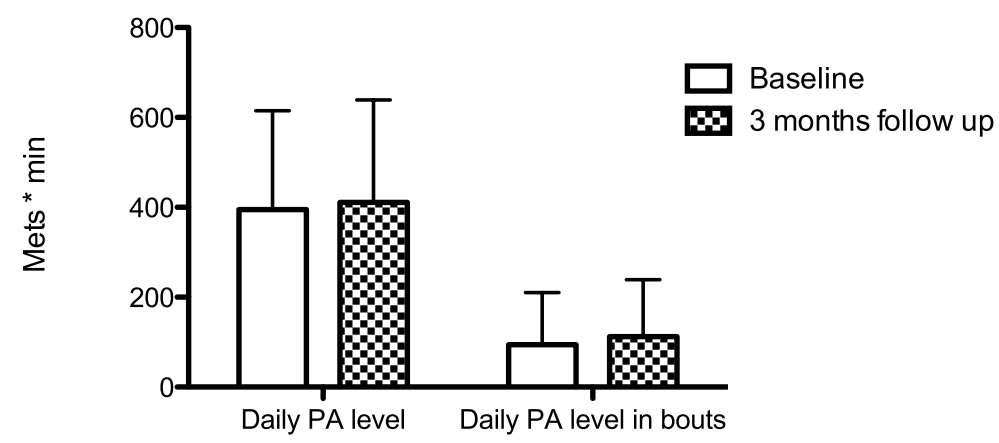

Note. Mets $=$ metabolic equivalents. ${ }^{*}$ Data were normally distributed and analyzed with a paired $t$ test. 


\section{Time consumption and intensity of various activities}

Table 2 depicts the time consumption and intensity of the various daily activities of IC patient. Data was non-normally distributed. No significant changes were found after 3 months of SET. Analysis of the intensity levels did also not show any significant differences between baseline and 3-months values (Table 2). However, the mean maximal number of consecutive steps (top period of steps), which was normally distributed, was significantly higher after 3 months of SET (1914 \pm 1473 vs. $2390 \pm$ 1904, p<.05).

\section{Walking distances and quality of life}

PFWD, MWD and total WIQ score significantly improved following SET (Table 3). Moreover, quality of life as reflected by SF-36 scores showed a significant increase in the physical functioning (PF) and the bodily pain (BP) role (Table 3). No significant correlation was found between the increase in both quality of life roles and an improvement in maximal walking distance ( $\triangle P F$ vs. $\triangle M W D: r=-.07, p=.75 ; \triangle B P$ vs. $\triangle M W D: r=.08, p=.73$ ).

TABLE 3 Quality of life and walking distances at baseline and at the 3 month follow up.

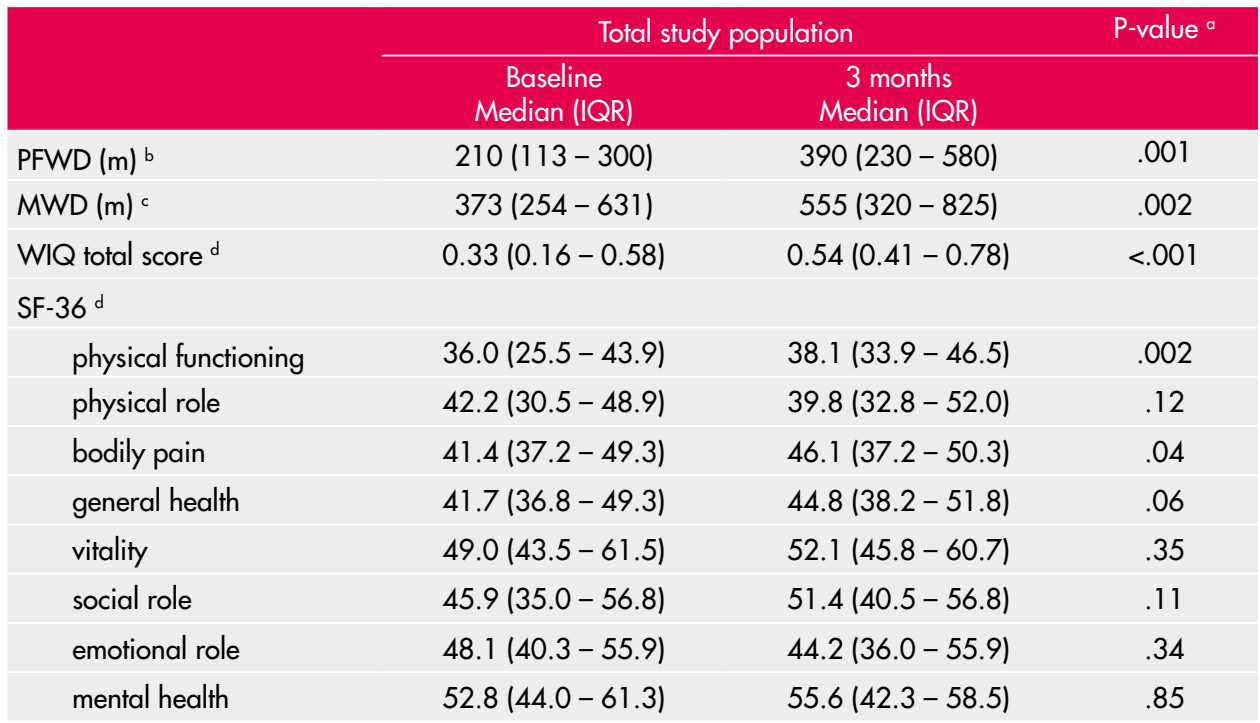

Note. Note. IQR = interquartile range; PFWD = pain free walking distance; $M W D=$ maximal walking distance; $\mathrm{WIQ}=$ Walking Impairment Questionnaire; SF-36 = Short Form Health Survey version 2.

a Values based on 34 patients. b Values based on 40 patients. c Values based on 39 patients. $d$ Wilcoxon signed rank test. 


\section{DISCUSSION}

In line with the upcoming interest in studying potential associations between daily PA and their impact on cardiovascular risk reduction and mortality ${ }^{35-41}$, this study assesses the effect of SET on proportions of IC patients meeting the ACSM lower and higher limit recommendation for PA and public health. Several studies registered activity levels in IC patients. ${ }^{15,17-21}$ However, this parameter was never considered as a primary outcome value. McDermott et al. showed in a 7-day measurement model that IC patients significantly improved their PA levels (in activity units) after 6 months of home-based exercise. ${ }^{21}$ These findings might in part be confirmed by the present results. Although our study was based on a shorter follow up period and based on supervised therapy, SET increased the percentage of IC patients achieving the lower and higher limit of daily PA level as recommended in the international ACSM guideline. " However, firm conclusions cannot be drawn, since some patients did show a decline in PA level and mean values for ambulatory activities (e.g. walking time, total number of steps) were not influenced after a period of SET. Probably large variations in activities per patient (duration as well as intensity) and between patients in combination with our relatively small study population could have caused this contradiction. Therefore further research is necessary to optimize the determination of PA levels in patients suffering from IC. However from our results it may be argued that a 3-month SET program may have beneficial effects on increasing daily PA in an IC population.

It was hypothesized that an increased walking capacity would result in an improved PA level of IC patients. Although both the percentage of patients meeting the ACSM limits and walking capacity significantly improved after 3 months of SET, no significant correlation between the change in PA levels and the increase in walking distance was found. Therefore one may argue that components of a SET program other than an increased walking capacity do influence daily PA. Our findings might suggest that solely focusing on improvement of walking capacity has a limited value in optimizing SET treatment efficacy. It appears that patients who are able to walk further (because of a decrease in IC symptoms), will not use this capacity to walk more often, longer or with probably more intensity in daily life.

The effect of SET on quality of life is still subject to debate. ${ }^{8} \mathrm{~A}$ recently published metaanalysis found a positive trend in improved quality of life after three months of SET, although only the SF-36 "general health" role appeared significantly increased. ${ }^{8}$ In addition, McDermott et al. found no group differences in changes in the SF-12 PCS or MCS subscales. ${ }^{21}$ In contrast, we found a significant increase in both the physical 
functioning and the bodily pain role after three months of SET. Although patients showed an increased walking capacity, no significant correlation in the change of SF36 values was found. This finding was recently confirmed by others reporting that the improvement in self-efficacy, satisfaction with functioning, pain acceptance and social functioning after six months of home-based walking exercise compared to a nonexercising control group was independent of walking performance. ${ }^{42}$ In combination with our finding that walking capacity might be of diminished value, a shift in SET treatment paradigm seems necessary. Future research should therefore focus on the optimization of SET programs other than increasing walking capacity alone (intrinsic motivation, life-style, patient satisfaction and expectations, social functioning, etc.).

Unfortunately a number of participants were lost to follow up which may have potentially resulted in a selection bias. However, this is probably not the case as any differences regarding baseline characteristics between the study group and excluded patients were found. Furthermore both study groups were a representative reflection of the general PAD population encountered in a vascular outdoor clinic. An important reason for this substantial loss to follow up is the fact that 56 of the 126 included patients were recruited from a previous performed study. ${ }^{16}$ Only 23 of these 56 patients were eligible for inclusion, because in the other patients the 3 months follow up period was already expired. Furthermore, introducing the Dynaport MoveMonitor from a research setting to daily practice in our patient outdoor clinic may boost wear compliance, as was observed in comparable research concerning COPD patients. ${ }^{43}$ Unfortunately, a reliable sub-analysis between revascularization and SET treatment could not be performed as only 16 participants did receive such a treatment. Future research is needed to identify the effect of different IC treatment modalities on daily PA levels and ambulatory activities.

\section{CONCLUSION}

A 3-month SET program increases the number of patients achieving the ACSM/AHA public health minimum recommendations for physical activity (PA). The general focus of increasing solely walking capacity seems ancillary to other elements of supervision. Further research is needed to determine the optimal content of a SET program. In concert with the interest in factors such as sedentary activities and cardiovascular event risks, exercise behavior and PA might be included as outcome parameter in future PAD research and eventually clinical practice. 


\section{REFERENCES}

1. Norgren L, Hiatt WR, Dormandy JA, Nehler $M R$, Harris KA, Fowkes FG. Inter-Society Consensus for the Management of Peripheral Arterial Disease (TASC II). J Vasc Surg 2007;45 Suppl S:S5-67.

2. Hirsch AT, Haskal ZJ, Hertzer NR, et al. ACC/ AHA 2005 guidelines for the management of patients with peripheral arterial disease (lower extremity, renal, mesenteric, and abdominal aortic): executive summary a collaborative report from the American Association for Vascular Surgery/Society for Vascular Surgery, Society for Cardiovascular Angiography and Interventions, Society for Vascular Medicine and Biology, Society of Interventional Radiology, and the ACC/AHA Task Force on Practice Guidelines (Writing Committee to Develop Guidelines for the Management of Patients With Peripheral Arterial Disease) endorsed by the American Association of Cardiovascular and Pulmonary Rehabilitation; National Heart, Lung, and Blood Institute; Society for Vascular Nursing; TransAtlantic Inter-Society Consensus; and Vascular Disease Foundation. J Am Coll Cardiol 2006;47:1239-312.

3. Layden J, Michaels J, Bermingham S, Higgins B, Guideline Development G. Diagnosis and management of lower limb peripheral arterial disease: summary of NICE guidance. Bmi 2012;345:e4947.

4. Bermingham SL, Sparrow K, Mullis R, et al. The Cost-effectiveness of Supervised Exercise for the Treatment of Intermittent Claudication. Eur J Vasc Endovasc Surg 2013;46:707-14.

5. Mazari FA, Khan JA, Carradice D, et al. Economic analysis of a randomized trial of percutaneous angioplasty, supervised exercise or combined treatment for intermittent claudication due to femoropopliteal arterial disease. Br J Surg 2013;100:1 172-9.

6. Spronk S, Bosch JL, den Hoed PT, Veen HF, Pattynama PM, Hunink MG. Costeffectiveness of endovascular revascularization compared to supervised hospital-based exercise training in patients with intermittent claudication: a randomized controlled trial. J Vasc Surg 2008;48:1472-80.

7. Fakhry $F$, van de Luijtgaarden $K M, B a x ~ L$, et al. Supervised walking therapy in patients with intermittent claudication. J Vasc Surg 2012;56:1132-42.
8. Fokkenrood $\mathrm{HJ}$, Bendermacher $\mathrm{BL}$, Lauret GJ, Willigendael EM, Prins MH, Teijink JA. Supervised exercise therapy versus nonsupervised exercise therapy for intermittent claudication. Cochrane Database Syst Rev 2013;8:CD005263.

9. Brach JS, FitzGerald $S$, Newman $A B$, ef al. Physical activity and functional status in community-dwelling older women: a 14year prospective study. Arch Intern Med 2003; 163:2565-71.

10. Nguyen $\mathrm{HQ}$, Ackermann RT, Maciejewski $M$, et al. Managed-Medicare health club benefit and reduced health care costs among older adults. Preventing chronic disease 2008;5:A14.

11. Nelson ME, Rejeski WJ, Blair SN, et al. Physical activity and public health in older adults: recommendation from the American College of Sports Medicine and the American Heart Association. Circulation 2007;1 16:1094-105.

12. Ainsworth BE, Haskell WL, Herrmann SD, ef al. 2011 Compendium of Physical Activities: a second update of codes and MET values. Med Sci Sports Exerc 2011 ;43:1575-81.

13. Gardner AW, Womack CJ, Sieminski DJ, Montgomery PS, Killewich LA, Fonong T. Relationship between free-living daily physical activity and ambulatory measures in older claudicants. Angiology 1998;49:327-37.

14. Sieminski DJ, Gardner AW. The relationship between free-living daily physical activity and the severity of peripheral arterial occlusive disease. Vasc Med 1997;2:286-91.

15. Crowther RG, Spinks WL, Leicht AS, Sangla K, Quigley F, Golledge J. Effects of a longterm exercise program on lower limb mobility, physiological responses, walking performance, and physical activity levels in patients with peripheral arterial disease. J Vasc Surg 2008;47:303-9.

16. Lauret GJ, Fokkenrood HJ, Bendermacher BL, Scheltinga MR, Teijink JA. Physical activity monitoring in patients with intermittent claudication. Eur J Vasc Endovasc Surg 2014;47:656-63.

17. Gardner AW, Montgomery PS, Parker DE. Optimal exercise program length for patients with claudication. J Vasc Surg 2012;55:134654. 
18. Gardner AW, Parker DE, Montgomery PS, Scott KJ, Blevins SM. Efficacy of quantified home-based exercise and supervised exercise in patients with intermittent claudication: a randomized controlled trial. Circulation $2011 ; 123: 491-8$.

19. Nicolai SP, Teijink JA, Prins MH. Multicenter randomized clinical trial of supervised exercise therapy with or without feedback versus walking advice for intermittent claudication. J Vasc Surg 2010;52:348-55.

20. HiattWR, Creager MA, Amato A, Brass EP. Effect of propionyl-L-carnitine on a background of monitored exercise in patients with claudication secondary to peripheral artery disease. Journal of cardiopulmonary rehabilitation and prevention $2011 ; 31: 125-32$

21. McDermott MM, Liu K, Guralnik JM, et al. Home-based walking exercise intervention in peripheral artery disease: a randomized clinical trial. Jama 2013;310:57-65.

22. Kodama S, Saito K, Tanaka S, et al. Cardiorespiratory fitness as a quantitative predictor of all-cause mortality and cardiovascular events in healthy men and women: a meta-analysis. Jama 2009;301:202435.

23. Yusuf S, Hawken S, Ounpuu S, et al. Effect of potentially modifiable risk factors associated with myocardial infarction in 52 countries (the INTERHEART study): casecontrol study. Lancet 2004;364:937-52.

24. Lauret GJ, Gijsbers HJ, Hendriks EJ, Bartelink ML, de Bie RA, Teijink JA. The ClaudicatioNet concept: design of a national integrated care network providing active and healthy aging for patients with intermittent claudication. Vasc Health Risk Manag 2012;8:495-503.

25. Jongert $M$, Hendriks $H$, van Hoek J, Kaasboer Kogelman K, Robeer G, Simens B. KNGF richtlijn claudicatio intermittens. . Nederlands Tijdschrift voor Fysiotherapie 2003:3-50.

26. Gardner AW, Skinner JS, Cantwell BW, Smith LK. Progressive vs single-stage treadmill tests for evaluation of claudication. Medicine and science in sports and exercise $1991 ; 23: 402-8$.

27. Jongert MWH, H.J.; van Hoek, J. [Guideline intermittent claudication from the Royal Dutch Society of Physical Therapy]. Ned Tijdschr Fysioth 2003;6:50.
28. Van Remoortel H, Raste Y, Louvaris Z, et al. Validity of six activity monitors in chronic obstructive pulmonary disease: a comparison with indirect calorimetry. PloS one 2012;7:e39198.

29. Fokkenrood HJ, Verhofstad N, van den Houten $M M$, et al. Physical activity monitoring in patients with peripheral arterial disease: validation of an activity monitor. Eur J Vasc Endovasc Surg 2014;48:194-200.

30. Dijkstra B, Kamsma Y, Zijlstra W. Detection of gait and postures using a miniaturised triaxial accelerometer-based system: accuracy in community-dwelling older adults. Age and ageing 2010;39:259-62.

31. Dijkstra B, Kamsma YP, Zijlstra W. Detection of gait and postures using a miniaturized triaxial accelerometer-based system: accuracy in patients with mild to moderate Parkinson's disease. Archives of physical medicine and rehabilitation. 2010;91:1272-7.

32. Verspaget $M$, Nicolai SP, Kruidenier $L M$, Welten RJ, Prins MH, Teijink JA. Validation of the Dutch version of the Walking Impairment Questionnaire. Eur J Vasc Endovasc Surg 2009;37:56-61.

33. Ware JE, Jr. SF-36 health survey update. Spine 2000;25:3130-9.

34. Ware JE, Jr., Gandek B. Overview of the SF36 Health Survey and the International Quality of Life Assessment (IQOLA) Project. Journal of clinical epidemiology 1998;51:903-12.

35. Grontved A, Hu FB. Television viewing and risk of type 2 diabetes, cardiovascular disease, and all-cause mortality: a meta-analysis. Jama $2011 ; 305: 2448-55$.

36. Katzmarzyk PT. Physical activity, sedentary behavior, and health: paradigm paralysis or paradigm shift? Diabetes 2010;59:2717-25.

37. Katzmarzyk PT. Standing and Mortality in a Prospective Cohort of Canadian Adults. Med Sci Sports Exerc 2013.

38. Maher C, Olds T, Mire E, Katzmarzyk PT. Reconsidering the sedentary behavior paradigm. PloS one 2014;9:e86403.

39. Thorp AA, Owen N, Neuhaus M, Dunstan DW. Sedentary behaviors and subsequent health outcomes in adults a systematic review of longitudinal studies, 1996-2011. American journal of preventive medicine 2011 ; 41:207-15. 
40. van der Ploeg HP, Chey T, Korda RJ, Banks E, Bauman A. Sitting time and all-cause mortality risk in 222497 Australian adults. Arch Intern Med 2012;172:494-500.

41. Wilmot EG, Edwardson CL, Achana FA, et al. Sedentary time in adults and the association with diabetes, cardiovascular disease and death: systematic review and meta-analysis. Diabetologia 2012;55:2895-905.
42. Rejeski WJ, Spring B, Domanchuk K, et al. A group-mediated, home-based physical activity intervention for patients with peripheral artery disease: effects on social and psychological function. Journal of translational medicine 2014;12:29.

43. Niessen $M$, Pijnappels $M$, van Dieën J, Van Lummel RC. Detecting not-wearing periods during activity monitoring in older adults. 2013. 


\section{CHAPTER 7}

\section{THE CLAUDICATIONET CONCEPT: DESIGN OF A NATIONAL INTEGRATED CARE NETWORK FOR PATIENTS WITH INTERMITTENT CLAUDICATION}

G.J. Lauret

H.J.H. Gijsbers

H.J.M. Hendriks

M.L. Bartelink

R.A. de Bie

J.A.W. Teijink

Vasc Health Risk Manag 2012;8:495-503 


\section{ABSTRACT}

\section{Introduction}

Intermittent claudication (IC) is a manifestation of peripheral arterial occlusive disease. Besides cardiovascular risk management, supervised exercise therapy (SET) should be offered to all patients with IC. Outdated guidelines, an insufficient number of specialised physiotherapists (PT's), lack of awareness of the importance of SET by referring physicians and misguided financial incentives, all seriously impede the availability of a structured SET programme in the Netherlands.

\section{Description of care practice}

By initiating regional care networks, ClaudicatioNet aims to improve the quality of care for patients with IC. Based on the chronic care model as conceptual framework, these networks should enhance the access, continuity and (cost-) efficiency of the health care system. With the aid of a national database, healthcare professionals will be able to benchmark patient results while ClaudicatioNet will be able to monitor quality of care by way of functional and patient reported outcome measures.

\section{Discussion}

The success of ClaudicatioNet is dependent on several factors. Vascular surgeons, general practitioners and coordinating central caregivers will need to team up and work in close collaboration with specialised PT's. A substantial task in the upcoming years will be to monitor the quality, volume and distribution of ClaudicatioNet PT's. Finally, misguided financial incentives within the Dutch Healthcare system need to be tackled.

\section{Conclusion}

With ClaudicatioNet, integrated care pathways are likely to improve in the upcoming years. This should result in achieving optimal quality of care for all patients with intermittent claudication. 


\section{INTRODUCTION}

Peripheral arterial occlusive disease (PAOD) is an expression of systemic atherosclerosis. Plaque formation causes progressive narrowing and hardening of the arteries in the legs. The manifestation of PAOD is variable, with some patients being asymptomatic and others developing tissue loss that ultimately necessitates amputation of the affected limb. 'The prevalence of PAOD is estimated to be $1.6 \%$ to $4.5 \%$ in the general population and as high as $19.1 \%$ in the Dutch population aged 55 years and older. ${ }^{2-6}$

Intermittent claudication $(I C)$, the mildest symptomatic manifestation of PAOD, is defined as pain in the muscles of the leg during exercise that is relieved by a short period of rest. IC is reported in $4.5 \%$ to $32.5 \%$ of all patients with PAOD. ${ }^{2,3,5}$ For IC, a five-year mortality rate of $19.2 \%$ is described, of which $70 \%$ is due to cardiovascular causes. Non-fatal cardiovascular events (e.g. myocardial infarction, stroke) in patients with IC are found in $29 \%$ at five years of follow-up. ${ }^{7}$ The prevalence of cerebrovascular disease in patients with IC is about $25-50 \% .{ }^{8}$

Numerous consensus documents provide recommendations for diagnosis and management of patients with PAOD, including the American College of Cardiology/ American Heart Association (ACC/AHA) and Trans-Atlantic Inter-Society Consensus on Management of Peripheral Arterial Disease (TASC II) guidelines on PAOD. 1,9 These guidelines identify patients with IC as a high-risk population who require intensive risk factor modification. Blood pressure levels, glucose, and cholesterol metabolism should all be optimised. Attention should be paid to smoking cessation since smoking is considered the most important modifiable vascular risk factor. Antiplatelet therapy and the use of a statin is indicated for all IC patients to reduce the risk of cardiovascular events. ${ }^{10,11}$

The primary symptomatic treatment for IC is exercise therapy, which was first suggested by Erb in 1898..$^{12-13}$ In a Cochrane review of systematic reviews, Watson et al. described an overall improvement in maximal walking time with a mean difference of 5.12 minutes ( $95 \%$ confidence interval $4.51-5.72$ ) in favour of exercise therapy compared with usual care or placebo. ${ }^{14}$ Exercise therapy consisted of all sorts of exercise prescriptions, including walking advice and unsupervised exercise regimes. Further benefit of exercise therapy was seen in the reduction of cardiovascular risk factors including hypercholesterolemia, hypertension, and diabetes mellitus. ${ }^{15}$ Given its clear benefits, the importance of exercise therapy is highlighted in contemporary international guidelines. ${ }^{1,9}$ 
In daily practice, lack of specific individual guidance and the absence of uniform supervision appear to be important barriers concerning the initiation and continuation of exercise therapy. ${ }^{16}$ For this reason, exercise programmes under supervision of a physiotherapist (PT) are considered beneficial. A Cochrane review by Bendermacher et al. showed that supervised exercise therapy (SET) was superior to a non-supervised exercise program. The maximum walking distance increased by an additional 150 meters in patients treated with SET compared to non-supervised exercise programmes. ${ }^{17}$ In a cohort study, community-based SET appeared to be as effective as hospital-based SET. ${ }^{18}$ Compared to outpatient hospital-based SET, community-based SET has the advantage of providing a larger capacity of care, in most cases being feasibly close to the patient's home environment with reduced transport costs. With this body of evidence, it is clear that SET should be offered eventually to all patients with IC.

\section{PROBLEM STATEMENT}

For the Dutch situation, there are several reasons as to why a structured SET programme is not offered to all patients with IC.

\section{Outdated guidelines of referring physicians}

In The Netherlands, the diagnosis of IC is determined by a general practitioner or a vascular surgeon. In all cases, the general practitioner (GP) has to initiate cardiovascular risk management according to the 2011 Dutch multidisciplinary guideline 'Cardiovascular Risk Management'. ${ }^{19}$ Both general practitioners as vascular surgeons are expected to start conservative symptomatic treatment for patients with IC, according to contemporary international guidelines for PAOD. 1,9 However, guidelines of the Dutch Association of General Practitioners and Dutch Society for Vascular Surgery are in an urgent need of an update since SET is not advocated as initial treatment for patients with IC. ${ }^{20,21}$

\section{Outdated arguments not to refer for a SET programme}

A quarter of new patients with IC, seen by a vascular surgeon, do not receive SET, as recently described in a national survey. ${ }^{22}$ The referral rate of GP's is unknown, although this will most probably be low given the small impact IC has on the general practice of a GP. Vascular surgeons who do refer for SET $(75,4 \%)$ mentioned arguments why not to refer for SET in certain cases. These arguments are: existing significant cardiopulmonary comorbidity; an aortoiliac stenosis or occlusion causing IC; and limited availability of sufficiently specialised PT's. ${ }^{22}$ Nevertheless, only the limited availability of PT's seems 
to be a valid argument. The remaining arguments appear to be outdated by current literature.

Furthermore, in case of severe or invalidating IC, invasive vascular interventions are often performed without first awaiting the effects of a structured SET programme. If a better infrastructure of specialised PT's exists, more patients would be able to receive SET from the onset.

\section{Referring to whom?}

If indications and referrals for SET have been set in a proper way, the next question is to whom should be referred? More than 22.000 PT's in the Netherlands should make it possible to provide SET according to the Royal Dutch Society for Physical Therapy guideline. ${ }^{23}$ However, the majority of these PT's are not adequately schooled in providing SET and do not have sufficient experience to provide this form of therapy. Patients with IC suffer from a variety of comorbidities and modifiable lifestyle factors, potentially generating suboptimal results if these aspects are not addressed appropriately. When adequately trained, PT's should ideally be able to perform individual training, recognise alarm symptoms (so called red and yellow flags) and even provide lifestyle counseling or monitor medication adherence. Volume criteria could have an influence on patient outcomes, since in general, more experience leads to better results. In order to achieve these better patient outcomes differentiation in the field of physical therapy is needed.

\section{Misguided financial incentives}

Not the least problem is the fact that the Dutch healthcare system does not stimulate use of conservative treatment for patients with IC, while invasive vascular interventions are fully compensated. The Dutch government determines the scope of coverage of the basic health care and health insurers are obligated to accept people for this insurance without any selection. For additional coverage to the basic health insurance package, a voluntary additional health care insurance can be acquired. Health insurers can determine the content and prices for their additional packages and can refuse acceptation for additional coverage for high-risk patients.

In this system, services provided by the GP or vascular surgeon, such as cardiovascular risk management or invasive vascular interventions, are fully compensated by the basic healthcare insurance. However, for physiotherapy, the first 20 treatment sessions of SET (for IC) are not covered by the basic health care insurance and have to be paid by the patient himself or by an additional insurance (since January 1st, 2012). All further treatment sessions, for a total of 1 year, are covered by the basic health care insurance. 
Therefore, in some cases, patients with IC have a financially driven interest to be treated by vascular surgeons who deliver invasive vascular interventions (for instance angioplasty or bypass surgery), in disregard of the associated risk of morbidity $(<0.5$ $10 \%$ ) and even mortality (2-3\% for bypass surgery). ${ }^{1}$

To tackle some of the aforementioned shortcomings, the ClaudicatioNet concept was developed and launched in the Netherlands in 2011.

\section{DESCRIPTION OF CARE INTERVENTION: CLAUDICATIONET}

The goal of the ClaudicatioNet programme is to improve the quality of care for patients with IC by creating connectivity and collaboration between the various players (including the patient) within the Dutch healthcare system. In general, by initiating regional care networks, ClaudicatioNet aims to enhance the access, continuity and (cost-) efficiency of the health care system. Furthermore, it aims to improve patient responsibility, patient satisfaction and quality of life for all patients with PAOD in the Netherlands.

\section{Initiation of the ClaudicatioNet concept}

The EXITPAD trial provided level lb evidence to support supervision in conjunction with exercise therapy for patients with IC. ${ }^{24}$ The concept of ClaudicatioNet has been based on the methods and results of this trial. In particular, many logistical efforts to make this trial possible provided useful knowledge for the initiation of the ClaudicatioNet concept.

ClaudicatioNet started in 2011 to initiate regional care networks between specially trained PT's, central caregivers and vascular surgeons. After initiation of a network, general practitioners are able to use the network's infrastructure. In regional networks, transparent, synergistic and multidisciplinary care has to be provided, based on the guidelines of cardiovascular risk management, SET and the Innovative Care for Chronic Conditions (ICCC) framework of the WHO.1,9,19,25 In addition to these evidence-based guidelines, the Dutch Health Care Inspectorate has developed (on behalf of the Ministry of Health, Welfare, and Sport) a set of mandatory key performance indicators. ${ }^{26}$ To meet these indicators, hospitals need to have a network of trained PT's capable of providing SET for patients with IC by 2012. 
The goal of ClaudicatioNet is to initiate the set up of regional networks throughout the Netherlands in a three years time period (January 2011 - December 2013). Since every network requires a three to four year period to expand to the desired level, full nationwide implementation should be achieved by 2016. The Dutch Society for Vascular Surgery, the Royal Dutch Society for Physical Therapy (KNGF), and the Dutch patient association for heart and vascular diseases (De Hart \& Vaatgroep) currently support ClaudicatioNet. With these partners, broad backing is in place to successfully implement this integrated care concept.

\section{Creation of care chains}

Ideally the care for PAOD patients is initiated by general practitioners and provided by health care professionals connected within horizontal primary care chains. In the Netherlands there are successfully operating and well-equipped primary care chains for diabetes and COPD that have proven to be able to deliver care conform national guidelines and care standards. However, at this moment the majority of the GP's do not participate in structured programs focusing on vascular risk management and do not take a leading or supervising role in the management of IC. There is an urgent need to solve this problem since a well-organised SET programme is stated in all contemporary guidelines and is listed as a key performance indicator by governmental bodies. To overcome the present care gap for patients with IC, a feasible and realistic solution had to be found. At this moment most patients are referred for SET by the 163 certified and practicing vascular surgeons in the Netherlands. A majority of these surgeons is motivated to contribute to the establishment of the ClaudicatioNet concept.

Therefore regional networks were chosen to set-up amongst vascular surgeons, central caregivers and PT's. After initiation, GP's will be able to use the network's infrastructure. In a region of the country where there is sufficient experience in setting up health care chains, further integration with three Dutch primary care health clusters (DOH, PoZoB, SGE) is being explored.

Both referring physicians and PT's benefit from participating in ClaudicatioNet. For referring physicians an infrastructure for care delivery with the use of specialised PT's becomes available. By only referring patients to PT's connected to ClaudicatioNet, vascular surgeons, general practitioners, and Health Care Insurers are guaranteed transparent and standardised high quality treatment for each patient. For vascular surgeons, a regional network is enrolled by ClaudicatioNet, which complies with the mandatory key performance indicator established by the Dutch Health Care Inspectorate. 
For PT's, ClaudicatioNet will provide a trajectory of specialisation in PAOD. This trajectory could be complementary to existing PT specialisations in the field of chronic illness or cardiopulmonary rehabilitation. Because of the growing need for differentiation in the field of physical therapy, participation will be a strategic choice for each individual PT or group practice. Furthermore, it is expected that the number of referrals per PT will increase significantly since only a proportion of all available PT's will participate in ClaudicatioNet and only these PT's will receive referrals. However, each PT has to make an economic trade-off between the potential profit and required investments derived from the mandatory participation criteria. How many referrals will lead to an acceptable profit giving the necessary investments is as yet uncertain. The answer pertaining to the viability of the business model of the program for primary care PT's is important with regard to the incentive to prolong future participation. Supply and demand of SET will evolve and reach equilibrium over time.

\section{Improving integrated care pathways by using the chronic care model}

The structure and working method of ClaudicatioNet is based on the internationally (WHO) accepted chronic care model that provides an evidence based, conceptual framework to improve the functional and clinical outcomes for people with chronic illness. The chronic care model describes the interacting system components which are important for providing good chronic illness care: self-management support; delivery system design; decision support and clinical information systems (Figure 1). ${ }^{27}$ The model also includes the organization of the health care system and other conditional factors like resources and policies.

ClaudicatioNet bridges the gap between different health care professionals (e.g. GP's and their practice assistants, vascular surgeons, specialised nurses in vascular surgery and PT's). Together they are able to provide the best standards of practice. Patients have a pivotal role in the care given. Specific information, treatment goals and obtained results need to be communicated to the patient. Many more treatment goals (lifestyle counseling, monitoring medication adherence) should ideally be reiterated and explained by all involved caregivers. However, the risk of health related information fatigue must be avoided. All of these elements ask for an integrated care approach. The chronic care model is used as a framework to improve the integrated care pathways and quality of care provided by all health care professionals (Figure 2). 
FIGURE 1. The Chronic Care Model

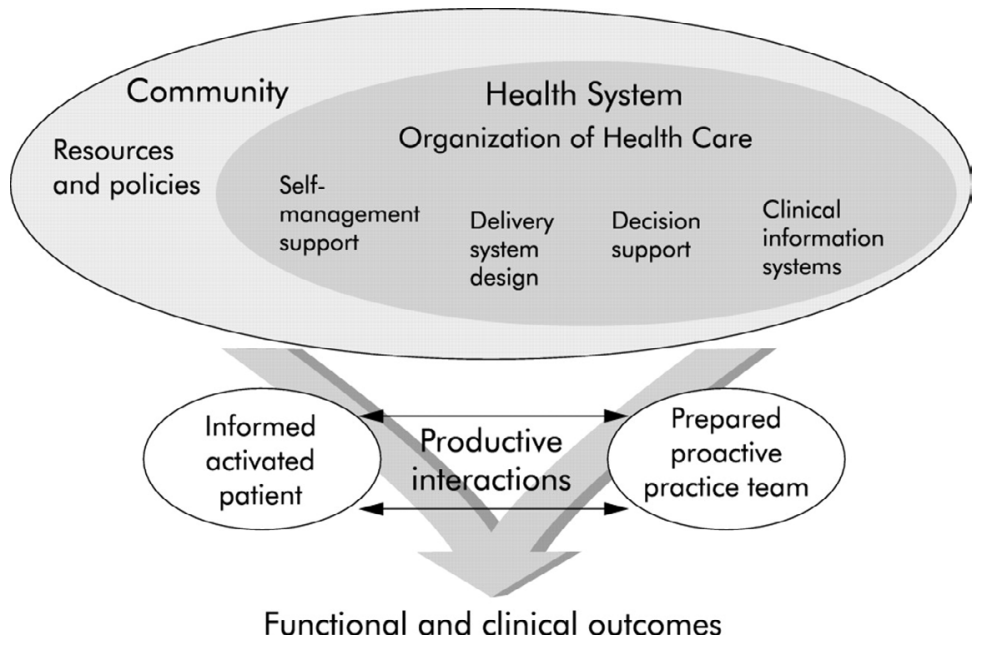

Note. adapted from Epping-Jordan et al. Qual Saf Health Care 2004

\section{Patients}

Patients are placed centrally in the care structure of the ClaudicatioNet concept, in which the patient his self-management is essential to yielding optimal patient outcomes. Health care professionals need to support and facilitate self-management and patient empowerment. In general, patients with IC have a low level of physical activity and in the majority of cases favour an unhealthy lifestyle (smoking, poor diet, lack of movement). An essential part of a successful SET program is self-initiated exercises between supervised training sessions.

In addition to the provided services of the involved health care professionals, ClaudicatioNet will provide supporting patient information through an interactive patient web portal. With the aid of this web portal patients are directed to background patient information regarding their disease, SET and/or lifestyle influences. Patients will be to able to find a specialised ClaudicatioNet PT in the online care finder and after initiation of SET, patients will have the possibility to monitor their own progress (based on individual data within the ClaudicatioNet database) in a user-friendly dashboard. Automatic reminders and the use of the interactive mobile application 'WalkMate' should increase adherence of patient self-management. With this app it will be possible to find, meet and train with other patients. 
FIGURE 2. Integrated care pathways

\section{GENERAL PRACTIONER}

- DIAGNOSING PAOD

- INITIATING CVRM

- DESIGNATING CENTRAL CAREGIVER

\section{VASCULAR SURGEON}

- DIAGNOSING PAOD

- INDICATING TREATMENT FORM (CONSERVATIVE / VASCULAR INTERVENTION / COMBINATION)

- designating CENTRAL CAREGIVER

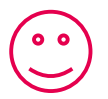

PATIENT

\section{CENTRAL CAREGIVER}

- SUPPLYING PATIENT INFORMATION (PAOD, CVRM, PHYSICAL THERAPY, LIFESTYLE CHANGES)

- COMPLETING STANDARD REFERRAL FORM

- COORDINATING CARE:

- CHOOSING PT WITH PATIENT

- COORDINATING NECESSARY APPOINTMENTS WITH HEALTH CARE PROFESSIONALS

- MONITORING OF PATIENT PROGRESS AND CONTACTING GP / VASCULAR SURGEON IN CASE OF DETORIATION

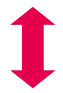

\section{PHYSICAL THERAPIST}

- treatMent ACCORDING ClaUdicAtionet QUALITY STANDARDS

- USING STANDARD FEEDBACK FORM (AT 3, 6 AND 12 MONTHS OF TRAINING)

- CONTACT WITH CENTRAL CAREGIVER IN CASE OF:

- NO THERAPY ADHERENCE

- PROGRESSION OF PAOD

- SUSPECTED DRUG NON-ADHERENCE

- SEVERE TRAINING LIMITATIONS DUE TO LIFESTYLE FACTORS / COMORBIDITY 
To evaluate the treatment provided by all the health care professionals, patients are asked to provide feedback on the number of treatment sessions, treatment protocol, facilities and expertise of individual health care professionals. Special attention will be paid to whether a patient receives training by a specialised PT.

\section{General practitioners and vascular surgeons}

General practitioners and vascular surgeons are the first caregivers to confront a patient with IC diagnosis and thus have the responsibility to diagnose compounding cardiovascular risk factors and attributing lifestyle factors. Ideally, GP's diagnose IC and initiate cardiovascular risk management while vascular surgeons determine if a vascular intervention is necessary. The guideline from the Dutch General Practitioners Society leaves the possibility to refer to a vascular surgeon in case of doubt or lack of available resources to perform an ABI (ankle-brachial index). In these cases it is a vascular surgeon whom initiates or advices first line treatment. After diagnosing and indicating treatment the patient should be designated to a central caregiver to coordinate the essential care for each individual patient. Ideally this takes place in primary care setting.

ClaudicatioNet necessitates that vascular surgeons only refer patients to a specialised ClaudicatioNet PT. Patients will be asked to provide feedback regarding the quality of care provided by the healthcare professional. Also, the number and origin of referrals will be monitored in the national ClaudicatioNet database and can, with the cooperation of health care insurers, be compared with the total number of patients with IC.

\section{Central caregiver}

Central caregivers (GP practice assistants or specialised vascular surgery nurses) play a major role in providing patient tailored management and coordination of given care from all involved caregivers. In an ongoing relationship with the patient, central caregivers provide integrated patient information regarding the condition, prognosis, influencing factors and treatment progress. Patients need to be supported in order to improve the management for their condition. Preferably, it is the patient and their central caregiver whom select a ClaudicatioNet PT. The central caregiver is responsible for the content and the process of the care given. He or she provides structure and follow-up according to the second component of the chronic care model.

Besides these tasks, central caregivers perform lifestyle interventions, monitor therapy adherence and initiate contact with the GP or vascular surgeon in case of detoriation 
of the patient his condition. Therefore, a central caregiver needs to work in close collaboration with all participating PT's in the regional network. In order to improve the system design, ClaudicatioNet developed standardised SET referral forms and patient leaflets with information regarding the interactive patient web portal. Central caregivers will be able to monitor the progress of their patients with a user-friendly web-based dashboard connected to the ClaudicatioNet database.

\section{Physiotherapists}

PT's participating in ClaudicatioNet perform standardised and evidence based SET, perform lifestyle interventions (e.g. smoking cessation, dietary advice) and regularly (2-4 times a year) assess medication adherence - all in close collaboration with the central caregiver. Prior to participating in a regional ClaudicatioNet network, PT's must meet the set inclusion criteria (Table 1).

\section{TABLE 1 Inclusion criteria for aspiring PT's}

Completed the course 'Intermittent Claudication', certified by the Royal Dutch Society for Physical Therapy.

Owns or works at a well equipped practice with a treadmill (with inclinometer), bicycle ergometer, reanimation protocol, automatic electronic defibrillator, internet connection, and yearly maintenance of equipment.

Broad oriented PT that recognises, reports, and acts on other limitations (like COPD, arthrosis, gait) that influence the patient's progress.

Prior to launching a regional network, a certified schooling programme on 'Intermittent Claudication', is provided by ClaudicatioNet for all aspiring PT's.

After inclusion, a PT is included in the online care-finder of ClaudicatioNet, which ideally should lead to a rise of the number of referrals. In a three year, step-wise program, PT's need to comply with increasing quality standards to ensure continued participation and referrals (Table 2). One additional year can be spent if the requirements of any given step in the specialisation process cannot be met. This results in a total of four years to obtain full specialization. After meeting the final criteria, PT's are considered to keep their knowledge up to date by attending the annual ClaudicatioNet congress once every two years and participating in lifelong learning activities through relevant schooling ( 15 dedicated study hours/year).

ClaudicatioNet provides an 'ask the expert' module, if a PT is confronted with a complex situation in their daily practice. This module is part of the ClaudicatioNet website and facilitates discussion about treatment difficulties with an expert PT and relates to the decision support component of the chronic care model. To gain efficiency in the delivery system design, ClaudicatioNet equips PT's with standard feedback 
forms. These forms provide essential information as inventoried in a national survey amongst vascular surgeons. ${ }^{22}$

TABLE 2 Participation criteria

\begin{tabular}{|c|c|c|}
\hline Step 1 & Step 2 & Step 3 \\
\hline $\begin{array}{l}\text { Standard care: delivers } \\
\text { transparent care according } \\
\text { to protocol, provides } \\
\text { feedback to vascular surgeon } \\
\text { and has adequate patient } \\
\text { administration. }\end{array}$ & $\begin{array}{l}\text { Practice visitation: complies } \\
\text { with quality indicators on } \\
\text { transparency and quality of } \\
\text { care, organisation of practice, } \\
\text { portfolio, and patient } \\
\text { administration. }\end{array}$ & $\begin{array}{l}\text { Patient Follow-up System: } \\
\text { works in the third year with a } \\
\text { patient follow-up system that } \\
\text { supplies data to the national } \\
\text { ClaudicatioNet database. }\end{array}$ \\
\hline $\begin{array}{l}\text { Schooling: attended } \\
\text { continuous medical education } \\
\text { (CME) on peripheral arterial } \\
\text { disease or concomitant } \\
\text { disease. In total, } 15 \text { credits } \\
\text { should be obtained each } \\
\text { year. The ClaudicatioNet } \\
\text { congress is mandatory every } \\
\text { two years. }\end{array}$ & $\begin{array}{l}\text { Schooling: attended } \\
\text { continuous medical education } \\
\text { (CME) on peripheral arterial } \\
\text { disease or concomitant } \\
\text { disease. In total, } 15 \text { credits } \\
\text { should be obtained each } \\
\text { year. The ClaudicatioNet } \\
\text { congress is mandatory every } \\
\text { two years. }\end{array}$ & $\begin{array}{l}\text { Schooling: attended } \\
\text { continuous medical education } \\
\text { (CME) on peripheral arterial } \\
\text { disease or concomitant } \\
\text { disease. In total, } 15 \text { credits } \\
\text { should be obtained each } \\
\text { year. The ClaudicatioNet } \\
\text { congress is mandatory every } \\
\text { two years. }\end{array}$ \\
\hline $\begin{array}{l}\text { Progress: completed an } \\
\text { electronic inquiry of all results } \\
\text { and number of patients. }\end{array}$ & $\begin{array}{l}\text { Portfolio: provided an } \\
\text { updated personal portfolio for } \\
\text { web use (care-finder module). }\end{array}$ & $\begin{array}{l}\text { Course: completed the course } \\
\text { 'Motivational Interviewing' } \\
\text { and is able to perform lifestyle } \\
\text { intervention for each patient }\end{array}$ \\
\hline
\end{tabular}

The progress of each individual PT will be monitored and if necessary acted upon. Quality evaluation based on a clinical information system is possible once PT's supply data (e.g. patient outcomes, number of treatment sessions, referral information) to the national ClaudicatioNet database. Recently a set of evidence based performance indicators has been developed. ${ }^{28}$ This data will be combined at regular intervals with measured feedback from patients (e.g. the number and content of training sessions, treatment provision by appropriate (certified) caregivers, satisfaction and perceived quality of care). With this data ClaudicatioNet will be able to monitor and improve future quality of care.

ClaudicatioNet promotes networking between health care professionals by organising regional network meetings with PT's, case managers, GPs and vascular surgeons. The goal of these meetings is to evaluate daily practice and exchange knowledge. ClaudicatioNet also organises an annual congress focusing on the management of PAOD. As part of the congress two parallel regional lunch programs, one specific for PT's and one aimed at case managers are organised. This lunch program enables 
both groups to discuss regional organisational aspects (for instance network meetings, regional schooling or other activities) and the coordination of regional care (for instance referral practice, quality of feedback).

\section{DISCUSSION}

Once ClaudicatioNet is operating nationwide, more patients with symptomatic PAOD, if not all, will benefit from standardised SET. This will lead to a reduced need for vascular interventions (percutaneous angioplasties or peripheral bypass operations). Morbidity and mortality due to these prevented interventions will cease to exist, resulting in a substantial population health gain. Prevention of costly interventions will also lead to a reduction of overall health costs. The promotion of life style activities by different caregivers as well as a structured, supervised and self-initiated, health stimulated exercise therapy will also have beneficial effects on the general health of patients. This, in turn, is likely to lead to reduced complications of existing comorbidities.

Health care insurers support this innovative concept and are willing to participate. A decline in the total number of vascular interventions has already been noted after the successful implementation of the first regional integrated care networks for IC. In the upcoming years, the number of patients admitted to ClaudicatioNet PT's will be compared to historical data and to the total number of vascular interventions. Patient outcomes will be gathered in the ClaudicatioNet database and compared to existing data. It is through these means that the success of ClaudicatioNet will be determined. In our opinion, the existence of a central caregiver as an easily accessible contact person within the first regional network, already improved patient communication and interaction between health care providers.

Success of ClaudicatioNet is, however, dependent on several factors. Vascular surgeons and GP's will need to refer patients with IC to PT's connected with ClaudicatioNet. Because ClaudicatioNet offers transparency of care with logistic support (referral packages, standardised feedback), ClaudicatioNet participation has evident advantages. Also, the mandatory key performance indicators of SET will help secure the collaboration of vascular surgeons to team up with ClaudicatioNet to launch a regional network. As mentioned previously, SET is not recommended in the current guidelines of the Dutch General Practitioners Society and Dutch Society for Vascular Surgery. Given the evidence provided in the current literature, adjustment of these guidelines is urgently needed to provide all patients with IC the best possible care. 
Another critical success factor is nationwide coverage. To achieve this goal a sufficient number of PT's will need to participate. Given the number of referrals and the supply and demand dynamics in each regional network, the volume of patients will determine the economic trade-off for the individual PT. When a PT chooses not to participate in ClaudicatioNet, more referrals are divided among the remaining PT's. ClaudicatioNet will not actively interfere with the geographic distribution of participating PT's. In the near future, supply and demand of SET will evolve and eventually create a balance within the market. In a worst-case scenario, regional coverage may not be fully achieved in some areas. Should this situation arise, ClaudicatioNet will subsequently try to recruit PT's to those specific areas.

Monitoring, updating and increasing the quality of ClaudicatioNet PT's will be a substantial task in the upcoming years and also an essential success factor. Participating PT's need to comply with a greater number of quality standards compared to nonparticipating PT's. It is necessary in our opinion to reward those PT's whom provide excellent care according to the established quality standards. Compensation for those PT's should be linear to the quality provided and, over time, Health Care Insurers should compensate only those PT's who deliver excellent care standards. To achieve this, a specific role needs to be played by Health Care Insurers. ClaudicatioNet will collaborate with these Health Care Insurers in order to accomplish this goal.

Another topic of discussion is the financial compensation for patients. In our opinion, every patientadmitted to ClaudicatioNet should be fully compensated for the conservative treatment programme by the mandatory Dutch basic health care insurance. In the current Dutch Health Care system, patients whom undergo a percutaneous vascular intervention or bypass surgery are fully compensated, in contrast to SET for which a financial contribution is still compulsory. To advocate this, we will argue for a system in which superior care is fully compensated compared to less-superior care. It is our objective to convince the Health Care Insurance Board (CVZ) of this necessity.

Future research should assess if the ClaudicatioNet concept is an effective approach for patients with critical limb ischemia whom have undergone a percutaneous vascular intervention or peripheral bypass surgery. For IC, the initiation of a cost-effectiveness study of the ClaudicatioNet concept will be the next step to determine the scope of efficiency. 


\section{CONCLUSION}

In the Netherlands, evidence based conservative treatment is not offered to all patients with IC. ClaudicatioNet was launched to improve the quality of care, access, continuity and (cost-) efficiency of the health care system. The chronic care model provides a conceptual framework of the structure and working method of ClaudicatioNet. With this care intervention, integrated care pathways are ought to be improved in the upcoming years. This should result in an optimal quality of care for all patients with intermittent claudication. 


\section{REFERENCES}

1. Norgren L, Hiatt WR, Dormandy JA, Nehler MR, Harris KA, Fowkes FG. Inter-Society Consensus for the Management of Peripheral Arterial Disease (TASC II). Journal of Vascular Surgery 2007 Jan;45 Suppl S:S5-67

2. Fowkes FG, Housley E, Cawood EH, Macintyre CC, Ruckley CV, Prescott RJ. Edinburgh Artery Study: prevalence of asymptomatic and symptomatic peripheral arterial disease in the general population. International Journal of Epidemiology 1991 Jun;20(2):384-92.

3. Murabito JM, Evans JC, Nieto K, Larson MG, Levy D, Wilson PW. Prevalence and clinical correlates of peripheral arterial disease in the Framingham Offspring Study. American Heart Journal 2002 Jun; 143(6):961-5.

4. Hooij JD, Stoffers HE, Kester AD, Rinskens $\mathrm{PE}$, Kaiser V. Risk factors and cardiovascular diseases associated with asymptomatic peripheral arterial occlusion disease. The Limburg PAOD Study. Scandinavian Journal of Primary Health Care 1998;16(3):177-82.

5. Meijer WT, Hoes AW, Rutgers D, Bots ML, Hofman A, Grobbee DE. Peripheral arterial disease in the elderly: The Rotterdam Study. Arteriosclerosis, Thrombosis and Vascular Biology 1998 Feb;18(2):185-92.

6. Stoffers $H E$, Rinkens $P E$, Kester $A D$, Kaiser $V$, Knottnerus JA. The prevalence of asymptomatic and unrecognized peripheral arterial occlusive disease. International Journal of Epidemiology 1996 Apr;25(2):282-90.

7. Leng GC, Lee AJ, Fowkes FG, et al. Incidence, natural history and cardiovascular events in symptomatic and asymptomatic peripheral arterial disease in the general population. Int $J$ Epidemiol 1996;25:1172-81

8. Criqui MH. Systemic atherosclerosis risk and the mandate for intervention in atherosclerotic peripheral arterial disease. Am J Cardiol $2001 ; 88: 43 J-7 J$

9. Hirsch AT, Haskal ZJ, Hertzer NR, Bakal CW, Creager MA, Halperin JL, et al. ACC/AHÁ 2005 Practice Guidelines for the management of patients with peripheral arterial disease (lower extremity, renal, mesenteric, and abdominal aortic): a collaborative report from the American Association for Vascular Surgery/ Society for Vascular Surgery, Society for Cardiovascular Angiography and Interventions, Society for Vascular Medicine and Biology, Society of Interventional Radiology, and the
ACC/AHA Task Force on Practice Guidelines (Writing Committee to Develop Guidelines for the Management of Patients With Peripheral Arterial Disease): endorsed by the American Association of Cardiovascular and Pulmonary Rehabilitation; National Heart, Lung, and Blood Institute; Society for Vascular Nursing; TransAtlantic Inter-Society Consensus; and Vascular Disease Foundation. Circulation 2006 Mar 21;113(11):e463-e654.

10. Antithrombotic Trialists Collaboration. Collaborative meta-analysis of randomised trials of antiplatelet therapy for prevention of death, myocardial infarction, and stroke in high risk patients. British Medical Journal 2002 Jan 12;324(7329):71-86.

11. Heart Protection Study Collaborative Group. Randomized trial of the effects of cholesterollowering with simvastatin on peripheral vascular and other major vascular outcomes in 20,536 people with peripheral arterial disease and other high-risk conditions. J Vasc Surg 2007 Apr;45(4):645-54.

12. Stewart KJ, Hiatt WR, Regensteiner JG, Hirsch AT. Exercise training for claudication. New England Journal of Medicine 2002 Dec 12;347(24):1941-51.

13. Erb W. Über das "intermittirende Hinken" and andere nervose Storungen in Folge von Gefasserkrankungen [About intermittent walking and nerve disturbances due to vascular disease]. Deutsche Zeitschrift für Nervenheilkunde 1898;13:1-76.

14. Watson L, Ellis B, Leng GC. Exercise for intermittent claudication. Cochrane Database of Systematic Reviews 2008;(4):CD000990.

15. Shephard RJ, Balady GJ. Exercise as cardiovascular therapy. Circulation $1999 \mathrm{Feb}$ 23;99(7):963-72.

16. Bartelink ML, Stoffers HE, Biesheuvel CJ, Hoes AW. Walking exercise in patients with intermittent claudication. Experience in routine clinical practice. British Journal of General Practitioners 2004 Mar;54(500): 196-200.

17. Bendermacher BL, Willigendael EM, Teijink JA, Prins MH. Supervised exercise therapy versus non-supervised exercise therapy for intermittent claudication. Cochrane Database of Systematic Reviews 2006;(2):CD005263. 
18. Kruidenier LM, Nicolai SP, Hendriks EJ, Bollen EC, Prins MH, Teijink JA. Supervised exercise therapy for intermittent claudication in daily practice. Journal of Vascular Surgery 2009 Feb;49(2):363-70.

19. Dutch Institute for Healthcare Improvement $C B O$ and Dutch College of General Practitioners Consensus Cardiovasculair Risicomanagement [Dutch Guideline Cardiovascular Risk Management], Utrecht, the Netherlands: Dutch Institute for Healthcare Improvement $\mathrm{CBO}$; 2011.

20. Bartelink ML, Stoffers HEJH, Boutens EJ, Hooi JD, Kaiser V, Boomsma U. NHG-standaard Perifeer arterieel vaatlijden [Guideline peripheral arterial disease from the Dutch General Practioners Society]. Huisarts en Wetenschap 2003;46:848-58.

21. Vahl AC, Reekers JA. Richtlijn 'Diagnostiek en behandeling van arterieel vaatlijden van de onderste extremiteit' van de Nederlandse Vereniging voor Heelkunde [The guideline 'Diagnosis and treatment of peripheral artery disease of the lower extremities' of The Netherlands Surgical Society]. Nederlands Tijdschrift voor Geneeskunde 2005 Jul 23;149(30):1670-4.

22. Lauret GJ, van Dalen HC, Hendriks HJ, van Sterkenburg SM, Koelemay MJ, Zeebregts CJ, et al. When is supervised exercise therapy considered useful in peripheral arterial occlusive disease? A nationwide survey among vascular surgeons. European Journal of Vascular and Endovascular Surgery 2012 Mar;43(3):308-12.
23. Jongert MW, Hendriks $H J$, van Hoek J, Klaasboer-Kogelman K, Robeer GC, Simens B. KNGF-richtliin Claudicatio Intermittens [Guideline intermittent claudication from the Royal Dutch Society of Physical Therapy], Ned T Fysiother 2003;6(supp): 1-50

24. Nicolai SP, Teijink JA, Prins MH. Multicenter randomized clinical trial of supervised exercise therapy with or without feedback versus walking advice for intermittent claudication. Journal of Vascular Surgery 2010 Aug;52(2):348-55.

25. World Health Organization. Innovative care for chronic conditions: building blocks for action. Geneva: World Health Organization; 2002.

26. Zichtbare Zorg Ziekenhuizen. [Indicator Set Chronic Peripheral Arterial Occlusive Disease] The Hague: Zichtbare Zorg Ziekenhuizen; 2011

27. Epping-Jordan EJ, Pruitt SD, Bengoa R, Wagner $\mathrm{EH}$. Improving the quality of health care for chronic conditions. Quality and safety in health care 2004 Aug;13(4):299-305

28. Gijsbers HJH, Lauret GJ, Hendriks HJM, Teijink JAW. Development of quality indicators for physical therapy in patients with PAOD. (submitted) 


\section{CHAPTER 8}

\section{DEVELOPMENT OF QUALITY INDICATORS FOR PHYSICAL THERAPY IN PERIPHERAL ARTERIAL OCCLUSIVE DISEASE}

H.J.H. Gijsbers

G.J. Lauret

A. van Hofwegen

R.A. de Bie

J.A.W. Teijink

Submitted; Physiotherapy 2015 


\section{ABSTRACT}

\section{Objectives}

The aim of the study was to develop quality indicators (Q/s) for physiotherapy management of patients with intermittent claudication (IC).

\section{Design}

As part of an international six-step method to develop Qls, an online survey Delphiprocedure was performed.

\section{Participants}

Twenty-six experts were recruited to participate in this study. Twenty-four experts completed the Delphi procedure.

\section{Results}

Outof83 potential QIs in the Dutch physiotherapyguideline on "Intermittentclaudication", consensus among the experts selected five indicators; 1) The physiotherapist measures the functional claudication distance and absolute claudication distance by performing a standardized treadmill test, 2) The outcome measures are assessed at baseline, after three months, six months, twelve months and at the end of the physiotherapy intervention, 3) The physiotherapist administers the Walking Impairment Questionnaire (WIQ), 4) The physiotherapist measures quality of life using the Euro Quality of Life-5D and 5)The physiotherapist checks non-compliance with medication use.

\section{Conclusion}

A final set of five indicators was derived from (1) an evidence-based guideline and (2) an expert Delphi procedure. This set of indicators should be validated before being implemented in clinical practice. 


\section{BACKGROUND}

The prevalence of peripheral artery occlusive disease (PAOD) in the general population of the Netherlands aged 55 years or older is $19.1 \%(\mathrm{Cl} 18.1-20.0)$. $^{1}$ Sociodemographic developments suggest an increase in the incidence and prevalence of PAOD in the next 15 years. PAOD implies an elevated risk of cardiovascular and cerebrovascular disease and higher mortality rates, as PAOD is a marker of generalised atherosclerotic disease. ${ }^{1-3}$

The five-year mortality associated with intermittent claudication is $19.2 \% .{ }^{4}$ When PAOD becomes symptomatic, patients often present with the typical symptoms of intermittent claudication (IC) such as "cramping," "fatigue," or "aching" in the calf of the leg, induced by walking and relieved by rest (1). IC is associated with a significantly lower quality of life, which can be improved with supervised exercise therapy (SET). ${ }^{5}$

Evidence for the efficacy of SET for patients with IC has evolved rapidly, with a Cochrane review and multiple randomised controlled trials of good quality. ${ }^{6-8}$ Compared to walking advice or endovascular revascularisation, SET showed favourable effects in terms of walking distances, functional impairment and health-related quality of life $6,9,10$, and seems to be cost-effective. " Community-based SET seems as efficacious as hospitalbased SET. ${ }^{9}$ The intensity and duration of treatment are well described. ${ }^{12}$

In the Netherlands, SET is reimbursed by health insurance in patients with IC if it is provided by a trained and skilled physiotherapist. In 2003, the Royal Dutch Society for Physical Therapy (KNGF) published an evidence-based guideline on physical therapy for IC for physiotherapists in the Netherlands. ${ }^{13}$ This guideline was distributed among the Society's members and is freely accessible on the internet. However, dissemination of guidelines does not guarantee sufficient implementation of each guideline or adherence to the (key) recommendations in clinical practice ${ }^{14-16}$, and the use of quality indicators $(Q \mid s)$ has been suggested in order to obtain a valid and appropriate measure of guideline adherence. ${ }^{17}$ European quality standards have been developed for cardiovascular risk prevention and risk management in primary care and comprise 44 indicators. ${ }^{18,19}$

However, QIs for the physical therapy management of IC are lacking.

This article reports on a research project for the development of Qls for physical therapy interventions in patients with IC. The aim of the study was to develop a feasible set of QIs for physical therapy management of IC. Opinions of experts were explored to identify aspects that are relevant and important for patients with IC. 


\section{METHODS}

Based on the guideline on "Intermittent claudication" of the Royal Dutch Society for Physical Therapy ${ }^{13}$, we developed a set of Qls, using the first four steps of an international six-step method. (Table 1) 17,22

TABLE 1 Steps in the process of developing QIs for PT for patients with IC.

Step 1. Selection of relevant patient group and care process.

Step 2. Deriving potential indicators from the national guideline

Step 3. Rating of the relevance of each potential indicator by an expert panel

Step 4. Operationalisation of prioritised indicators into draft set of quality indicators.

Step 5. Practice-testing the initial set of indicators using clinical vignettes

Step 6. Establishing definitive indicator set

\section{Deriving potential indicators from the guideline}

Two authors (HG, EH) drew up a list of potentially suitable indicators from the national guideline recommendations and the literature.

\section{Rating the relevance of each potential indicator}

Two of the authors (HG, EH) selected and invited experts (12 physiotherapists, 12 vascular surgeons and residents, 3 lecturers) for the expert panel. Expert physiotherapists were physiotherapists with over 5 years of experience in providing SET, working in primary care as well as in hospitals, and complying with the quality standards of the Dutch integrated care network "ClaudicatioNet". The invited vascular surgeons and trainee vascular surgeons knew the effects of SET. The invited lecturers were experienced in teaching physiotherapists about intermittent claudication or conducting research into this disease.

The experts were invited to participate in a two-stage online survey Delphi procedure, and were asked to rate the potential indicators in terms of relevance for the quality of physical therapy intervention. They could also add further potential indicators, provided these were thoroughly substantiated. The Qls were rated on a nine-point Likert scale from 1 to 9, where 1 meant "not relevant at all" and 9 meant "very relevant". After the first Delphi round, all potential indicators with a mean score $>8$ and a median score of 8 or 9 were presented in a second Delphi round, in which the experts remained blinded to the scores from the first Delphi round. The experts were invited to rate the indicators again on the same nine-point Likert scale, in order to obtain a feasible set of indicators. The experts were given the option of re-including indicators discarded from 
the original set. After these two stages, the remaining indicators with a mean score $>8$ or a median score of 8 or 9 were scored in a third Delphi round. Four experts of the expert panel with specific knowledge in measurability, were also asked to score the measurability of the remaining items on a 4-point scale (very relevant, relevant, some relevance, not relevant at all).

\section{Operationalisation of prioritised indicators into a draft set of Qls}

Potential indicators emerging from the third Delphi round, which had been unanimously rated as very relevant or relevant by all four experts were included in the draft set. Good measurability of the indicators was the main requirement for inclusion in the final draft set of indicators. All experts were subsequently asked to endorse this final draft set of indicators.

\section{Data analysis}

The cut-off values for the relevance of indicators from the Delphi procedure were determined using the Kolmogorov-Smirnov test to check for a normal distribution. In case of normal distribution, indicators with a mean score $>8$, as judged by the experts, were selected as relevant. If the distribution was not normal, items with a median score of 8 or 9 on the nine-point Likert scale were selected as relevant indicators. This analysis was used for the first two Delphi rounds. In the third round, only items with a score of "very relevant" or "relevant" were selected.

\section{RESULTS}

Eighty-three potential Qls were initially derived from the recommendations of the evidence-based guideline on "Intermittent Claudication" of the Royal Dutch Society for Physical Therapy. An expert panel of 27 physical therapists, vascular surgeons and researchers were asked to rate the relevance of the indicators and suggest further indicators. This round was completed by 26 experts (for the characteristics of the expert panel see Table 2). All ratings were normally distributed, and 27 of the 83 indicators were selected in this first Delphi round.

After this first round, the same experts were asked to rate the relevance of the 27 indicators again. This round was completed by 24 experts (12 PTs, 9 VSs and 3 lectures). Fourteen of the 27 indicators then remained, and 11 of the indicators discarded in round one were re-included in the set of indicators. A total of 13 indicators were selected in this second Delphi. 
In the third Delphi round, two expert physical therapists and two expert vascular surgeons scored the indicators in terms of measurability. Only the indicators that were rated as "very relevant" or "relevant" were adopted as a new draft set of indicators. This reduced the number of indicators to five (Table 3). A systematic representation of the entire Delphi process is provided in Figure 1.

FIGURE 1. Overview of Delphi rounds and methods

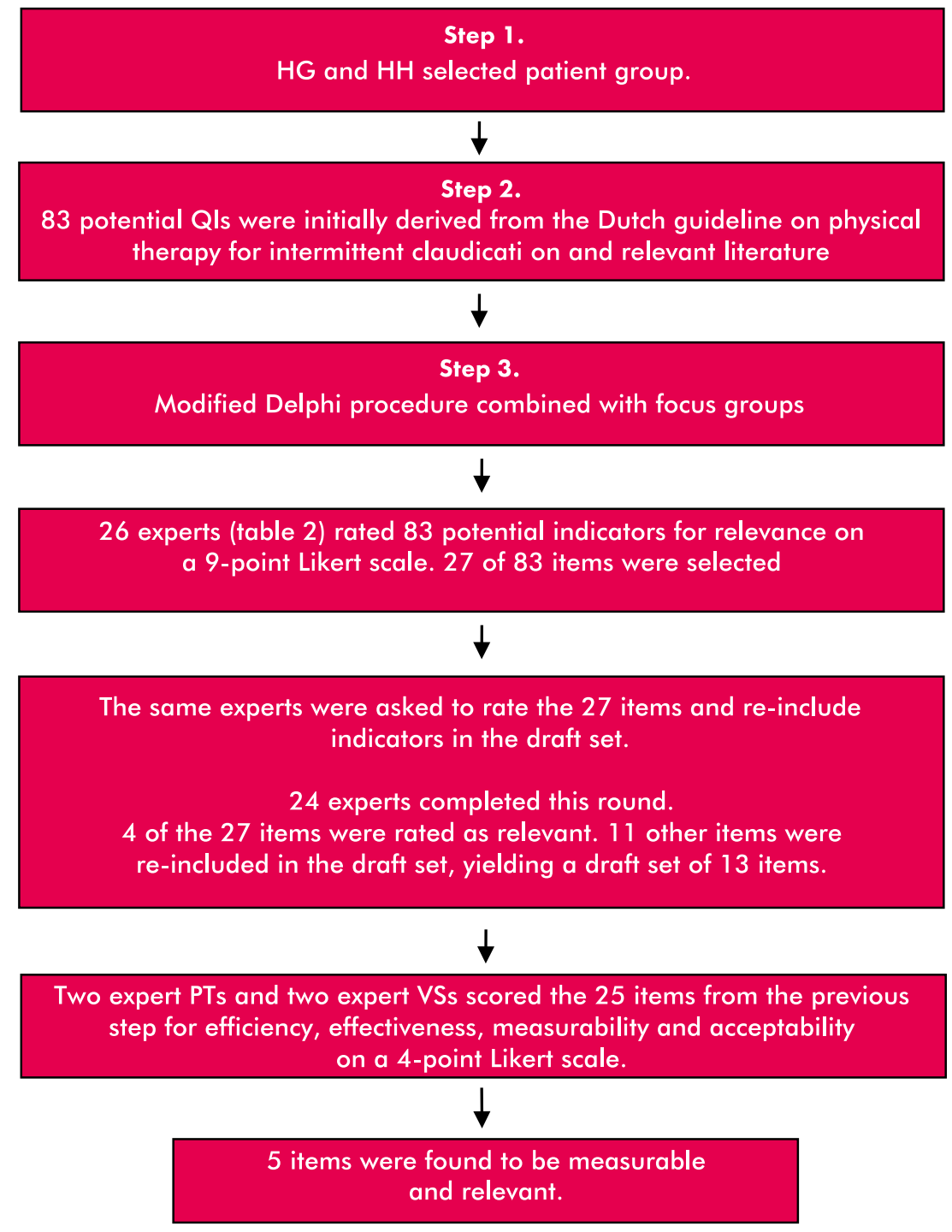


TABLE 2 Characteristics of expert panel

\begin{tabular}{|c|c|c|c|}
\hline & $\begin{array}{l}\text { PTs } \\
(n=12)\end{array}$ & $\begin{array}{l}\text { VSs and trainees } \\
(n=11)\end{array}$ & $\begin{array}{l}\text { Other } \\
(n=3)\end{array}$ \\
\hline Age (mean (range)) & $48(30-64)$ & $39.5(27-49)$ & $53(48-57)$ \\
\hline Work setting & $\begin{array}{l}7 \text { in primary care } \\
4 \text { in hospital }\end{array}$ & $\begin{array}{l}\text { Hospital or } \\
\text { research } \\
\text { department }\end{array}$ & $\begin{array}{l}\text { Academic, } \\
\text { lecturer }\end{array}$ \\
\hline $\begin{array}{l}\text { Years of experience with PAOD } \\
\text { (mean (range)) }\end{array}$ & $20.2(6-37)$ & $8.7(0-20)$ & $24.7(10-34)$ \\
\hline Number of patients in last 3 months & $9.8(0-40)$ & $86(0-250)$ & 0 \\
\hline
\end{tabular}

Note. PTs=physiotherapists, VSs=vascular surgeons

TABLE 3 Set of five quality indicators that resulted from three Delphi rounds.

\begin{tabular}{|c|c|c|}
\hline $\mathrm{Nr}$ & Indicator & Comments \\
\hline 1 & $\begin{array}{l}\text { The physiotherapist measures the } \\
\text { functional claudication distance and } \\
\text { absolute claudication distance by } \\
\text { performing a standardised treadmill test. }\end{array}$ & \\
\hline 2 & $\begin{array}{l}\text { The outcome measures are assessed at } \\
\text { baseline, after three months, six months } \\
\text { and twelve months and at the end of the } \\
\text { physical therapy intervention. }\end{array}$ & \\
\hline 3 & $\begin{array}{l}\text { The physiotherapist administers the } \\
\text { Walking Impairment Questionnaire (WIQ) }\end{array}$ & \\
\hline 4 & $\begin{array}{l}\text { The physiotherapist measures quality of life } \\
\text { using the Euro Quality of Life- } 5 D\end{array}$ & \\
\hline 5 & $\begin{array}{l}\text { The physiotherapist checks non-compliance } \\
\text { with medication use }\end{array}$ & $\begin{array}{l}28.5 \% \text { of the experts regarded this as very } \\
\text { relevant, but measurability is moderate. } \\
\text { Nevertheless, it was considered important } \\
\text { as a quality indicator }\end{array}$ \\
\hline
\end{tabular}

\section{DISCUSSION}

This is the first report on a research project for the development of Qls for physical therapy intervention in patients with IC, for which Qls were so far lacking. In accordance with international standards for the development of Q/s ${ }^{17,22}$ potential indicators were initially derived from an evidence-based guideline, while further potential indicators could be added in an expert Delphi procedure. This resulted in a set of five Qls, three of which were derived from the guideline, while two were added by the experts. 
The indicators "The physiotherapist administers the Walking Impairment Questionnaire (WIQ)" and "The physiotherapist measures quality of life using the Euro QoL-5D (EQ$5 D)^{\prime \prime}$ were added by the experts, thoroughly substantiated, in the first Delphi round. These two indicators were rated as relevant in the second and third Delphi rounds. The indicators "The physiotherapist checks non-compliance with medication use" and "The physiotherapist supports smoking cessation" were added in the first Delphi round and were rated as very relevant in the second round. In the third Delphi round, however, the indicator "The physiotherapist supports smoking cessation" was assessed as poorly measurable, so it was initially excluded.

\section{Comparison with other quality indicator sets for cardiovascular disease}

A direct comparison of the set of Qls proposed here with similar sets of Qls for physical therapy management of IC is not possible, as to our knowledge, this is the first publication describing Qls for physical therapy for IC. However, a comparison can be made with Qls for cardiovascular risk management, as Ludt et al. described the EPAcardio instrument, which measures the quality of cardiovascular risk management in European primary care. ${ }^{19}$ This instrument includes patient questionnaires about physical activity, lifestyle, eating and smoking habits. The EPA-cardio instrument corresponds to some extent with the lifestyle indicators presented in our study.

A multidisciplinary guideline for vascular risk management was developed in the Netherlands in 2006. This guideline is based on the chronic care model. ${ }^{21}$ The indicator "The physiotherapist checks non-compliance with medication use" in the present study is in line with this multidisciplinary guideline, in that it endorse the need for good interdisciplinary communication and well-informed patients.

\section{Comparison with other quality indicators for physical therapy}

Until now, Qls to assess adherence with the recommendations of the Dutch physical therapy guidelines have only been systematically developed for the guidelines on Parkinson's disease ${ }^{28}$ and osteoarthritis of the hip and knee. ${ }^{29}$ These Qls were selected in a procedure involving experts in a Delphi procedure, similar to that employed in the present study.

\section{Limitations}

This study was subject to certain limitations. In the process of formulating potential indicators, the experts did not take into account the level of evidence underlying the recommendations. As a result, recommendations based on single studies and expert 
opinion (levels 3 and 4) were also included. It is a matter of debate whether a minimum level of evidence is required for recommendations to be included in sets of Qls. Another limitation is the fact that we derived indicators from the evidence-based guideline on "Intermittent claudication" of the Royal Dutch Society for Physical Therapy, which is based on literature up to 2002. Physical therapy research in PAOD is evolving rapidly and new evidence since then has been mainly found to favour SET, making the recommendations from the 2003 guideline even stronger. We believe that our use of the expert Delphi procedure has resulted in an up-to-date set of indicators.

Another weakness of this study might be the measurability of some indicators. The expert group was aware of the poor measurability of some indicators, and therefore excluded some indicators like "Offers a prognosis", "Provides good aftercare", "supports smoking cessation" and "promotes an active lifestyle".

In a demand-driven or client-centered care system, services should meet the patients' needs and preferences. ${ }^{20,21}$ Our study did not focus on quality assurance (e.g. through Qls) from the patient's perspective.

\section{Implications for further research}

More research is needed to validate our proposed set of indicators. This could be done by checking randomly sampled patient files, using case vignettes or developing a questionnaire incorporating the indicators from the set. ${ }^{17,} 28$ In order to describe content-validity it is important to clarify also what the patients' needs are and what these needs imply for the development of QIs for physical therapy in the treatment of IC. The clinical implications of this study seem very promising, as the use of this set of quality indicators enables further examination of adherence to the guideline for physical therapy for patients with IC.

\section{CONCLUSION}

A set of five quality indicators was derived from an evidence-based guideline, and an expert Delphi procedure. This set of indicators should be validated before it can be used for implementation in clinical practice. 


\section{REFERENCES}

1. Meijer WT, Hoes AW, Rutgers D, Bots ML, Hofman A, Grobbee DE. Peripheral arterial disease in the elderly: The Rotterdam Study. Arterioscler Thromb Vasc Biol. 1998; 18(2):18592.

2. Criqui $M H$, Denenberg JO, Langer RD, Fronek A. The epidemiology of peripheral arterial disease: importance of identifying the population at risk. Vasc Med. 1997;2(3):221-6.

3. Fowkes FG, Housley E, Cawood EH, Macintyre CC, Ruckley CV, Prescott RJ. Edinburgh Artery Study: prevalence of asymptomatic and symptomatic peripheral arterial disease in the general population. Int J Epidemiol. $1991 ; 20(2): 384-92$

4. Leng GC, Lee AJ, Fowkes FG, Whiteman $M$, Dunbar J, Housley $E$, et al. Incidence, natural history and cardiovascular events in symptomatic and asymptomatic peripheral arterial disease in the general population. Int J Epidemiol. 1996;25(6):1172-81.

5. Spronk S, White JV, Bosch JL, Hunink MG. Impact of claudication and its treatment on quality of life. Semin Vasc Surg. 2007;20(1):39.

6. Bendermacher BL, Willigendael EM, Teijink JA, Prins $M H$. Supervised exercise therapy versus non-supervised exercise therapy for intermittent claudication. Cochrane Database Syst Rev. 2006(2):CD005263.

7. Spronk S, Bosch JL, den Hoed PT, Veen HF, Pattynama PM, Hunink MG. Intermittent claudication: clinical effectiveness of endovascular revascularization versus supervised hospital-based exercise training-randomized controlled trial. Radiology. 2009;250(2):586-95

8. Murphy TP, Cutlip DE, Regensteiner JG, Mohler ER, Cohen DJ, Reynolds MR, et al. Supervised exercise versus primary stenting for claudication resulting from aortoiliac peripheral artery disease: six-month outcomes from the claudication: exercise versus endoluminal revascularization (CLEVER) study. Circulation. 2012;125(1):130-9.

9. Kruidenier LM, Nicolai SP, Hendriks EJ, Bollen EC, Prins MH, Teijink JA. Supervised exercise therapy for intermittent claudication in daily practice. J Vasc Surg. 2009;49(2):363-70.
10. Nicolai SP, Teijink JA, Prins MH. Multicenter randomized clinical trial of supervised exercise therapy with or without feedback versus walking advice for intermittent claudication. J Vasc Surg. 2010;52(2):348-55.

11. van Asselt $A D$, Nicolai SP, Joore MA, Prins $M H$, Teiijink JA. Cost-effectiveness of exercise therapy in patients with intermittent claudication: supervised exercise therapy versus a 'go home and walk' advice. Eur J Vasc Endovasc Surg. 2011;41(1):97-103

12. Nicolai SP, Hendriks EJ, Prins MH, Teijink JA. Optimizing supervised exercise therapy for patients with intermittent claudication. J Vasc Surg. 2010;52(5):1226-33.

13. Jongert MWA, Hendriks HJM, Hoek Jv, Klaasboer-Kogelman K, Robeer GG, Simens B, et al. KNGF-richtlijn Claudicatio intermittens. 2003 [cited 113 6]; suppl].

14. van der Wees PJ, Hendriks EJ, Jansen MJ, van Beers $\mathrm{H}$, de Bie RA, Dekker J. Adherence to physiotherapy clinical guideline acute ankle injury and determinants of adherence: a cohort study. BMC Musculoskelet Disord. 2007;8:45.

15. Rebbeck T, Maher CG, Refshauge KM. Evaluating two implementation strategies for whiplash guidelines in physiotherapy: a cluster randomised trial. Aust J Physiother. 2006;52(3): 165-74.

16. van der Wees PJ, Jamtvedt $G$, Rebbeck $T$, de Bie RA, Dekker J, Hendriks EJ. Multifaceted strategies may increase implementation of physiotherapy clinical guidelines: a systematic review. Aust J Physiother. 2008;54(4):233-41.

17. Campbell SM, Braspenning J, Hutchinson A, Marshall MN. Research methods used in developing and applying quality indicators in primary care. BMJ. 2003;326(7393):816-9.

18. Campbell SM, Ludt S, Van Lieshout J, Boffin N, Wensing $M$, Petek $D$, et al. Quality indicators for the prevention and management of cardiovascular disease in primary care in nine European countries. Eur J Cardiovasc Prev Rehabil. 2008;15(5):509-15.

19. Ludt S, Campbell SM, van Lieshout J, Grol R, Szecsenyi J, Wensing M. Development and pilot of an internationally standardized measure of cardiovascular risk management in European primary care. BMC Health Serv Res. $2011 ; 11: 70$. 
20. Ham $C$. The ten characteristics of the highperforming chronic care system. Health Econ Policy Law. 2010;5(Pt 1):71-90.

21. Wagner EH, Bennett SM, Austin BT, Greene $S M$, Schaefer JK, Vonkorff M. Finding common ground: patient-centeredness and evidencebased chronic illness care. J Altern Complement Med. 2005;11 Suppl 1:S7-15.

22. Wollersheim $H$, Hermens $R$, Hulscher $M$, Braspenning J, Ouwens $M$, Schouten J, et al. Clinical indicators: development and applications. Neth J Med. 2007;65(1):15-22.

23. Miegchelbrink F. Praktijkgericht onderzoek in zorg en welzijn. Amsterdam: SWP; 2000.

24. Webb C, Kevern J. Focus groups as a research method: a critique of some aspects of their use in nursing research. J Adv Nurs. 2001;33(6):798805.

25. Kidd PS, Parshall MB. Getting the focus and the group: enhancing analytical rigor in focus group research. Qual Health Res. 2000;10(3):293308.

26. Kitzinger J. Qualitative research. Introducing focus groups. BMJ. 1995;311(7000):299-302.
27. Hendriks HJM, Oostendorp RA, Bernards ATM, Ravensberg CDv, Heerkens YF, Nelson RM. The diagnostic process and indication for physiotherapy: a prerequisite for treatment and outcome evaluation. Phys Ther Reviews. 2000(5):29-47.

28. Nijkrake MJ, Keus SH, Ewalds $H$, Overeem $\mathrm{S}$, Braspenning JC, Oostendorp RA, et al. Quality indicators for physiotherapy in Parkinson's disease. Eur J Phys Rehabil Med. 2009;45(2):239-45.

29. Jansen MJ, Hendriks EJ, Oostendorp RA, Dekker $J$, De Bie RA. Quality indicators indicate good adherence to the clinical practice guideline on "Osteoarthritis of the hip and knee" and few prognostic factors influence outcome indicators: a prospective cohort study. Eur J Phys Rehabil Med. 2010;46(3):337-45.

30. Collins TC, Kroll TL, Krueger PN, Willson $P$, Ashton CM, Sharf BF. A qualitative approach to developing a patient-derived intervention to increase exercise in peripheral arterial disease. J Cardiopulm Rehabil. 2006;26(2):92-100. 

CHAPTER 9

GENERAL DISCUSSION 



\section{MAIN FINDINGS}

\section{Limited availability and reluctance of SET}

Although contemporary guidelines clearly prescribe supervised exercise therapy (SET) as a first-line treatment in intermittent claudication (IC), many patients do not receive SET. ${ }^{1-4}$ The reason for this is multifactorial and lies with the doctor, who should prescribe SET as a first line treatment, the healthcare insurer, who should reimburse SET as a firstline treatment, and the patient, who should be willing to comply with SET as a first-line treatment. Other possible factors that contribute to the limited referral rate of SET are limited SET availability, misinterpretation of potential SET indications, patient resistance, professional self-interest and issues regarding the reimbursement of SET by the patient's healthcare insurance plan. ${ }^{4}$

In this thesis, we presented the results of a cross-sectional survey study that assessed the current opinion of Dutch vascular surgeons and fellows in vascular surgery regarding SET as a treatment option for IC. We specifically wanted to find out to what extent and on which criteria Dutch vascular surgeons decide to offer or deny SET to IC patients. Participants received a 25-question survey, which they could complete either during the Annual Meeting of the Dutch Society for Vascular Surgery or on a secure website. We showed that the respondents would refer $75 \%$ of newly diagnosed patients with IC for SET. Although the study had a response rate of $51 \%$ of all Dutch vascular surgeons, the results could have been influenced by responder bias, most likely in favor of a positive attitude towards SET. Therefore, the estimated three quarters of IC patients that were referred for SET may in fact be less in real life. A study on 2009 invoice data of a Dutch healthcare insurance company with 3.4 million members showed that only $14 \%$ of IC patients received SET, whereas $28 \%$ were primarily treated with an invasive intervention. The remaining $58 \%$ did not receive either of these two treatment regimens within 3 months of diagnosis. ${ }^{5}$

These results were also confirmed in a study on the use of SET in the UK. This study found a large spread in the proportion of patients referred for SET. ${ }^{3}$ Furthermore, only $24 \%$ of UK surgeons had access to SET. In the Netherlands, with over 20,000 physical therapists, community-based availability of SET is not a problem. However, the question is whether each physical therapist is capable of providing SET according to the guidelines of the Royal Dutch Society for Physical Therapy. To ensure optimal SET availability, continuity, cost-efficiency and quality of care, ClaudicatioNet was initiated in the Netherlands. This integrated care network ought to improve both access to and quality of SET. 
Our study showed that respondents did not refer for SET in the presence of significant cardiopulmonary comorbidity and aortoiliac stenosis or occlusion causing IC, since they did not consider SET to be safe for these indications. However, these arguments for not referring for SET seem to be outdated. In both cardiac and pulmonary rehabilitation programs, patients with congestive heart failure and chronic obstructive pulmonary disease undergo supervised aerobic exercise (e.g. treadmill walking and cycling) in a moderate to severe intensity. ${ }^{6-12}$ Also, exercise rehabilitation is considered safe for coronary heart disease, leading to a decreased 5-year all-risk mortality rate. ${ }^{9-12}$ SET can safely be prescribed given its exceedingly low all-cause complication rate. Routine cardiac screening is not required before commencing SET. ${ }^{13}$ Therefore, there is no reason why SET should not be offered to patients with coexistence of IC and congestive heart failure or chronic pulmonary or coronary conditions. Nonetheless, physical therapists should adjust patient monitoring and exercise intensity to each patient's condition.

There are several explanations why SET is not as effective in aortoiliac disease as endovascular revascularisation. First, patients with aortoiliac disease have more ischemic muscle mass while walking, and consequently they are more often symptomatic than those with a more distal obstruction. ${ }^{14}$ Therefore, patients with aortoiliac disease might experience less improvement with exercise training. Second, surgeons have considerably more experience with stent revascularization in the aortoiliac segment, and the results are more predictable and durable than those observed in the femoropopliteal artery segment. ${ }^{15-19}$ However, results demonstrate that for aortoiliac disease, SET resulted in a superior treadmill walking distance after 6 months compared to percutaneous transluminal angioplasty (PTA) with stent revascularization, even though a nonsignificant improvement of disease-specific quality of life was found in favor of PTA. ${ }^{20}$ With this evidence, there is no reason why SET should not be offered to patients with IC caused by aortoiliac disease. Also, from a practical standpoint, initiation of SET should not be delayed by diagnostics of disease location, since there is no evidence that this has any consequences for the initiation of primary conservative treatment in IC.

Another possible explanation for the limited use of SET could be patient resistance. Many IC patients want a quick solution for their problem. Therefore they prefer a swift vascular intervention over lifestyle changes and a lengthy exercise program. Vascular surgeons may try to convince patients of the benefits of SET. However, if a patient refuses, a vascular intervention is readily available. This in turn is closely related to the final obstacle for referring for SET, which is professional self-interest. Vascular surgeons are remunerated for the execution of interventions. They opt for these invasive treat- 
ments in the conviction that they optimally serve their patients, but at the same time this decision is in line with their professional self-interest, since vascular interventions lead to higher reimbursements than SET. Moreover, invasive treatments are opportunities to train or improve skills. Also, vascular interventions are encouraged by the medical industry's emphatic promotion of new invasive techniques, whereas SET lacks promotion.

The reluctance to refer for SET may also be explained by the fact that the conservative treatment for patients with IC is not stimulated by the Dutch health care system. In this system, the scope of coverage for basic health care is determined by the Dutch government, and health insurers are obligated to accept applicants for this insurance without any selection. For additional coverage a voluntary additional health care insurance can be acquired, but the content and prices for these additional packages are determined by health insurers, who can refuse to accept high-risk patients who apply for additional coverage.

Under the current coverage for basic health care, some patients with IC have a financially driven interest to refrain from SET and to be treated by invasive vascular interventions, in disregard of the associated risks of morbidity or mortality. Basic health care insurance fully compensates services provided by the general practitioner or vascular surgeon, such as cardiovascular risk management or invasive vascular interventions. However, for IC, the first 20 treatment sessions of SET are not covered by basic health care insurance and have to be paid by the patient himself or by an additional insurance. Basic health care insurance does cover all further treatment sessions, for a total of 1 year. This provision suggests that the value of SET for IC patients is officially recognized, but it does not benefit patients who are unable to afford the first 20 sessions of SET.

The ideal situation for both the patient and the healthcare system would be a steppedcare approach, in which SET is prescribed first and vascular intervention is only applied in case of SET failure. Implementation of such a stepped-care model may lead to a significant annual saving up to 33 million euro of healthcare resources in the Netherlands. Hence, healthcare insurers should invest in this stepped-care program. Vascular surgeons should restrict vascular interventions to cases of SET failure, and should be reimbursed accordingly. Further cost-efficiency could be achieved if general practitioners coordinate conservative treatment and only refer patients in need of vascular intervention. This can be achieved by more integrated collaboration with vascular laboratories and vascular surgeons in regional care networks. ClaudicatioNet provides a clear framework to create nationwide coverage of these regional integrated care networks. 
As this thesis showed, the causes of the limited availability of SET and the reluctance to refer for SET are multifactorial and require a drastic change in the views of general practitioners, vascular surgeons, healthcare insurers and patients in order to optimize both the quality and the cost-efficiency of healthcare for IC patients. More attention should be paid to evaluating the quality of SET. Furthermore, individual patients might benefit from patient-tailored exercise modes.

\section{Modes of exercise training}

A number of potential mechanisms have been suggested that may explain why exercise (in general) might benefit patients with IC. Reduced functional capacity in IC might be due to blood flow limitation caused by arterial obstruction, disruption of endothelial function, altered skeletal muscle phenotype by mitochondrial dysfunction, increased blood viscosity, and inflammatory activation. ${ }^{21}$ Exercise, independent of its mode, has the potential to reverse these pathological processes and thus to interrupt the clinical course toward disability. Animal models of arterial insufficiency indicate that exercise training augments peripheral arterial supply. ${ }^{22-25}$ In rodent models, exercise stimulated gains in collateral blood flow after femoral occlusion through collateral enlargement. $22,26,27$ Collateral growth induced by exercise reflects vascular structural remodeling, a process that depends on both growth factor activity and increased nitric oxide bioavailability via shear stress stimulation of endothelial nitric oxide synthase. $22,26,28$ In contrast to animal models, studies in patients with IC have not convincingly demonstrated that exercise training produces clinically relevant gains in peripheral blood flow. A recent meta-analysis of 7 exercise training studies demonstrated no change in the resting ankle-brachial index, a cumulative measure of blood supply to the lower extremities. ${ }^{29}$

As has been observed in coronary arteries, endothelial dysfunction could also lead to peripheral arterial vasoconstriction and limit vasodilator responses to flow, which would tend to exacerbate blood flow limitation during exercise. ${ }^{30-32}$ Consistent with these potential links between vascular function and functional status, a number of studies have demonstrated impaired endothelial vasodilator responses in IC. SET increased endothelium-dependent flow- mediated dilation of the brachial artery by $65 \%$ in elderly patients with IC. ${ }^{33}$ The capability of exercise to reverse endothelial dysfunction may reflect sustained increases in shear-stress that stimulate nitric oxide bioactivity. A study in patients with coronary disease showed that exercise rehabilitation induced favorable effects on coronary endothelial function which were associated with increased endothelial nitric oxide synthase expression and activation. ${ }^{34}$ As impaired endothelial function predicts higher risk for cardiovascular events among patients with IC, the 
exercise-induced improvement in vasodilator function may have the potential to reduce cardiovascular risk. ${ }^{35,36}$

Altered skeletal muscle energetics in IC has been linked to mitochondrial dysfunction. Intermediate metabolites of substrate oxidation, including acylcarnitines, accumulate in the blood and muscles of IC patients and are consistent with impaired metabolism at the mitochondrial level. ${ }^{37}$ Abnormal mitochondrial function may interfere with skeletal muscle oxygen utilization and may accelerate endothelial damage. ${ }^{38,39}$ Exercise training has the potential to enhance skeletal muscle metabolism and mitochondrial function. In IC patients, exercise training has been shown to restore carnitine metabolism in association with improved treadmill walking. ${ }^{37,40}$ Whether exercise training improves mitochondrial energy production or calf muscle characteristics in IC remains to be evaluated.

Furthermore, inflammation may accelerate functional impairment in IC by favoring plaque growth and inducing skeletal muscle injury. Endothelial inflammatory activation reduces nitric oxide bioavailability and may impede vasodilatory function during exercise. ${ }^{41}$ Physical activity may have favorable effects on IC by suppressing inflammatory activation. While acute bouts of exercise increase inflammatory markers in claudicants, chronic exercise training appears to curb inflammation. ${ }^{42} \mathrm{~A} 3$-month exercise program including treadmill exercise ameliorated neutrophil activation in patients with IC. ${ }^{43}$ It remains to be determined whether reduced inflammation produced by chronic exercise training underlies increased walking ability and translates to decreased events.

In a systematic review we assessed the effects of different exercise modes. The five included RCTs described only three alternative exercise modes: strength training, arm ergometry, and cycling exercise. Although data were limited, we showed that alternative modes of exercise therapy seem to yield results similar to those of supervised walking therapy. Therefore, these alternative modes may be considered useful for some groups of patients with IC who are not able to complete the exercise protocol because of concomitant comorbidities, such as arthrosis, chronic obstructive pulmonary disease, stroke, congestive heart failure or coronary artery disease.

Our systematic review was limited by the small sample size of 135 participants. However, sensitivity analysis did not significantly alter the results of this review. We tried to limit all the potential biases in the review process. In general, the risk of bias of the included studies was low, reflecting the good methodological quality of the included studies. We could not detect publication bias, because the number of studies was too 
limited to assess asymmetry in a funnel plot. To limit bias and make a meaningful comparison, we standardised the maximum walking distance and the pain-free walking distance in each study by converting walking distances to total metabolic equivalents according to the American College of Sports Medicine (ACSM) formulas for metabolic calculations. ${ }^{44}$ However, direct conversion of the walking times or distances to metabolic equivalents was not possible due to the absence of individual participant data. We therefore simulated a new dataset for each study to make a meaningful comparison. It is unclear to what extent this could have biased our findings.

More studies are needed to make meaningful comparisons between alternative exercise modes. Also, future research should clarify the potential mechanisms of different alternative exercise modes. In general, more randomized controlled trials of adequate size are needed to compare the different conventional and alternative exercise regimes to the standard of supervised walking exercise. Besides reporting walking performance, future trials should focus on the effect of the exercise mode on health-related quality of life. Exercise training studies in IC have most often used treadmill walking time or distance as the primary outcome. The use of such an objective measure may be of great importance to investigators and to clinicians, given its relationship to IC symptoms. However, a patient may judge the benefit of an intervention by its effects on health-related quality of life, which is composed of many dimensions and not just related to leg symptoms. The concept of health-related quality of life as a primary outcome has clearly been adopted in the IRONIC trial. ${ }^{45}$ Nevertheless, in standard clinical practice, quality of life is yet to be integrated as a tool to measure the clinical success of any intervention in patients with IC.

\section{Physical activity monitoring}

We conducted a prospective observational cohort study to assess the daily physical activity (PA) level of IC patients and healthy adults. We showed that more than half of the patients with IC did not meet the minimum recommendations for PA and public health. Compared with healthy adults, they had a significantly lower daily PA level. IC patients reporting substantial functional impairment (WIQ score < 0.4 ) had the lowest mean daily PA level of all groups. Interestingly, the PA group difference was solely attributed to this subgroup. Apparently, a lower daily PA level does not occur in IC patients who only have minor functional impairments.

The outcomes of this study may have been influenced by incorrect monitoring, which could be due to lack of motivation or failure to understand the monitoring protocol. In our monitoring protocol, participants were asked to remove the device before sleeping 
to improve compliance. In a few instances, participants fell asleep with the device still strapped to their waist. If the device was worn during the night, that day was marked by definition as "not correctly worn". We are not sure if compliance could have been higher if participants had been instructed to wear the device constantly. Also, a substantial number of participants said they had forgotten the end date of the monitoring period. For clinical practice, a shorter monitoring period could be considered. However, the accuracy of PA results from a shorter monitoring period should first be validated, since these results could vary.

A proportion of the cohort described in our study on monitoring PA was also included in a new study to determine the effect of SET on the daily PA level. We demonstrated that a 3-month SET program raised the number of patients who met the ACSM/AHA public health minimum recommendations for PA. The general focus of only increasing walking capacity seems ancillary to other elements of supervision. Exercise behavior and PA might be included as outcome parameters in future IC research and eventually in clinical practice. Unfortunately, a number of participants were lost to follow-up, which may potentially have resulted in a selection bias. However, bias was most likely limited, since no baseline differences were found between the study group and excluded patients. Furthermore, both study groups constituted a representative reflection of the general IC population encountered in a vascular outpatient clinic. Unfortunately, it was not possible to perform a reliable sub-analysis between revascularisation and SET treatment, as only 16 participants received a revascularisation.

In patients with IC, a low daily PA level is generally considered a strong predictor of mortality and functional decline. ${ }^{46-48}$ For this reason, the PA level could be viewed as a valid outcome parameter for evaluating the treatment effects of any intervention for IC. Current outcome parameters including maximum or pain-free walking distances only describe the patient's exercise capacity, while it does not reflect to what extent this capacity is used in daily life. In contrast, daily PA levels provide objective information on the frequency, duration and intensity of exercise. In combination with a functional impairment score, such as the Walking Impairment Questionnaire (WIQ) score, measuring daily PA levels enables physicians to objectively monitor the patient's progress during treatment. Therefore, future studies should include an objectively measured daily PA level as an additional outcome measure for IC. If proven to provide reliable and reproducible evidence, measuring daily PA levels might be a valid instrument to evaluate treatment indications and treatment progress in clinical practice. 


\section{Quality indicators}

We described the use of an online Delphi-procedure to develop quality indicators for physiotherapy management. This is the first report on a research project for the development of quality indicators for physical therapy in patients with IC. In accordance with international standards for the development of quality indicators, potential indicators were initially derived from an evidence-based guideline, while further potential indicators could be added in an expert Delphi procedure. ${ }^{49,50}$ Three quality indicators were derived from the guideline 'Intermittent Claudication' of The Royal Dutch Society for Physical Therapy, while two were added by the experts..$^{51}$ This resulted in a set of five quality indicators: 1) The physiotherapist measures the functional claudication distance and absolute claudication distance by performing a standardized treadmill test; 2) The outcome measures are assessed at baseline, after three months, six months and twelve months, and at the end of the intervention; 3) The physiotherapist administers the WIQ; 4) The physiotherapist measures quality of life using the Euro Quality of Life-5D; 5) The physiotherapist checks whether patients comply with the prescribed use of medication. This set of indicators should be validated before being implemented in clinical practice.

\section{IMPLICATIONS FOR CLINICAL PRACTICE AND FUTURE RESEARCH}

In this thesis we described to what extent and on which criteria (Dutch) vascular surgeons decide whether or not to refer IC patients for SET. Arguments not to refer for SET seem to be outdated. SET, including lifestyle coaching, should receive more emphasis in the current management of IC.

Clearly, some groups of patients with IC are not able to complete the exercise protocol because of concomitant comorbidities, such as arthrosis, chronic obstructive pulmonary disease, stroke, or cardiac complaints. For these patients, an adjusted protocol or alternative exercise regime may be proposed. In this thesis we tried to answer the question which exercise mode produces the most beneficial results. A systematic review of studies on alternative modes of exercise therapy summarised the reported results of cycling, strength training, and upper-arm ergometry. We showed that these alternative modes of exercise therapy seem to yield results similar to those of supervised walking therapy. Therefore, these alternative modes may be considered useful if supervised walking exercise is not an option for the patient. However, few studies included in our review were found to be of good quality. More randomised controlled studies with larger sample sizes are needed to make meaningful comparisons between each alternative exercise mode and the current standard of supervised treadmill walking. 
In this thesis we used the newest generation of accelerometers to objectively assess the daily PA level of patients with IC. Results demonstrate that less than half of these patients met the current minimal PA recommendations. The quantified daily level of PA in IC was significantly lower than in healthy adults. Because a low PA level in IC is considered a strong predictor of mortality and functional decline, this paper emphasizes the need for more awareness of improving physical exercise in patients with IC.

Furthermore, we describe the effect of SET on walking capacity, walking behavior, ambulatory activities and daily physical activity in IC patients. Results of this study demonstrate the promising results of measuring the daily PA level of IC patients with tri-axial accelerometry. PA can be considered a valid outcome parameter in evaluating treatment effects of any intervention for IC. Current outcome parameters including maximum- or pain-free walking distances only describe the patient' $s$ exercise capacity, while it does not reflect to what extent this capacity is used in daily life. In contrast, daily PA levels provide objective information on the frequency, duration, and intensity of exercise. Combined with a functional impairment score, such as the WIQ score, measuring daily PA levels enables physicians to objectively monitor the patient's progress during treatment. Future clinical studies on IC should focus on this relevant new outcome parameter. Furthermore, future studies should examine the effects of different treatment strategies on the daily PA level in IC.

In this thesis we describe the design of ClaudicatioNet, an integrated care network that aims to improve the quality of care for patients with IC by initiating regional care networks. Based on the chronic care model as a conceptual framework, these networks should enhance the access, continuity, efficiency and cost efficiency of the health care system. ClaudicatioNet bridges the gap between various health care professionals, such as general practitioners and their practice assistants, vascular surgeons, specialised nurses in vascular surgery, and physical therapists. Together they are able to provide the best standards of practice. Patients play a pivotal role in the care that is given. Specific information, treatment goals, and obtained results need to be communicated to the patient. Many more treatment goals (lifestyle counseling, monitoring of medication adherence) should ideally be explained and reiterated by all involved caregivers. All these elements indicate the need for an integrated care approach.

The success of ClaudicatioNet is dependent on several factors. Vascular surgeons, general practitioners and coordinating central caregivers will need to team up and work in close collaboration with specialized physical therapists. A substantial task in the upcoming years will be to monitor the quality, volume, and distribution of ClaudicatioNet 
physical therapists. Finally, misguided financial incentives within the Dutch health care system need to be tackled.

In this thesis we described the development of quality indicators for physiotherapy management in patients with IC. Consensus among experts selected five potential quality indicators. More research is needed to validate our proposed set of indicators. This could be done by checking randomly sampled patient files, using case vignettes or developing a questionnaire incorporating the indicators from the set. In order to describe content-validity, it is also important to clarify what the patients' needs are and what these needs imply for the development of quality indicators for physiotherapy in the treatment of IC. After validation, these quality indicators could be used as an instrument to measure quality of physiotherapy management of IC in the Netherlands. With the initiation of ClaudicatioNet, clinical implications of this study seem very promising, as the use of this set of quality indicators enables quality evaluation and examination of adherence to the guidelines for physiotherapy regarding patients with IC.

Additional future studies on IC should focus on how to manage patients with IC due to specific disease locations. For IC due to femoropopliteal disease, PTA and SET are found equally effective in improving walking distance and quality of life at a follow-up of 12 months. ${ }^{52}$ For aortoiliac disease, SET resulted in a superior treadmill walking distance after 6 months compared to PTA, while PTA achieved a non-significantly greater improvement of disease-specific quality of life. ${ }^{53}$ However, combining both treatments resulted in significantly better results in walking distance and disease specific quality of life for mixed aortoiliac and femoropopliteal disease, indicating a possible adjuvant benefit of combining both treatments. ${ }^{54-56}$ More research should confirm these findings and identify a possible difference between femoropopliteal and aortoiliac disease. Furthermore, costs of interventions can also play a decisive role in choosing the optimal intervention for patients with IC. Future research should also focus on the costeffectiveness of different treatment protocols for patients with IC, with a particular focus on a stepped-care protocol indicating the use of SET as first treatment option and PTA only in case of SET failure. 


\section{REFERENCES}

1. Norgren L, Hiatt WR, Dormandy JA, Nehler MR, Harris KA, Fowkes FG. Inter-Society Consensus for the Management of Peripheral Arterial Disease (TASC II). J Vasc Surg. 2007;45(Suppl S):S5-67.

2. Hirsch AT, Haskal ZJ, Hertzer NR, Bakal CW, Creager MA, Halperin JL, et al. ACC/AHA 2005 Practice Guidelines for the management of patients with peripheral arterial disease. Circulation. 2006;113(11):e463-654.

3. Shalhoub J, Hamish M, Davies AH. Supervised exercise for intermittent claudication $e$ an under-utilised tool. Ann R Coll Surg Engl. 2009;91(6):473-6.

4. Popplewell MA, Bradbury AW. Why Do Health Systems Not Fund Supervised Exercise Programmes for Intermittent Claudication? Eur $J$ Vasc Endovasc Surg. 2014;48(6):608-610.

5. Fokkenrood HJ, Scheltinga MR, Koelemay MJ, Breek JC, Hasaart F, Vahl AC, et al. Significant savings with a stepped care model for treatment of patients with intermittent claudication. Eur $\mathrm{J}$ Vasc Endovasc Surg. 2014;48(4):423-9.

6. Casaburi R, ZuWallack R. Pulmonary rehabilitation for management of chronic obstructive pulmonary disease. N Engl J Med. 2009;360(13):1329-35.

7. Ries AL, Bauldoff GS, Carlin BW, Casaburi $\mathrm{R}$, Emery CF, Mahler DA, et al. Pulmonary rehabilitation: Joint ACCP/AACVPR Evidencebased Clinical Practice Guidelines. Chest. 2007;131(5 Suppl.). 4S-42S.

8. Verrill D, Barton C, Beasley W, Lippard WM. The effects of short-term and long-term pulmonary rehabilitation on functional capacity, perceived dyspnea, and quality of life. Chest. 2005;128(2):673-83.

9. Suaya JA, StasonWB, Ades PA, Normand SL, Shepard DS. Cardiac rehabilitation and survival in older coronary patients. J Am Coll Cardiol. 2009;54(1):25-33.

10. Jessup $M$, Abraham WT, Casey DE, Feldman AM, Francis GS, Ganiats TG, et al. 2009 focused update: ACCF/AHA Guidelines for the diagnosis and management of heart failure in adults: a report of the American College of Cardiology Foundation/American Heart Association Task Force on Practice Guidelines: developed in collaboration with the International Society for Heart and Lung Transplantation. Circulation. 2009;119(14):1977-2016.
11. O'Connor CM, Whellan DJ, Lee KL, Keteyian SJ, Cooper LS, Ellis SJ, et al. Efficacy and safety of exercise training in patients with chronic heart failure: HF-ACTION randomized controlled trial. J Am Med Assoc. 2009;301(14):143950.

12. Van Camp SP, Peterson RA. Cardiovascular complications of outpatient cardiac rehabilitation programs. J Am Med Assoc. 1986;256(9): 1160-3.

13. Gommans LN, Fokkenrood HJ, van Dalen HC, Scheltinga MR, Teiijink JA, Peters RJ. Safety of supervised exercise therapy in patients with intermittent claudication. J Vasc Surg. 2014;pii: S0741-5214(14)01636-X.

14. Pernow B, Zetterquist $S$. Metabolic evaluation of the leg blood flow in claudicating patients with arterial obstructions at different levels. Scand J Clin Lab Invest. 1968;21:277-287.

15. Murphy TP, Ariaratnam NS, Carney WI Jr., Marcaccio EJ, Slaiby JM, Soares GM, Kim HM. Aortoiliac insufficiency: long-term experience with stent placement for treatment. Radiology. 2004;231:243-249.

16. Sapoval MR, Long AL, Raynaud AC, Beyssen BM, Fiessinger JN, Gaux JC. Femoropopliteal stent placement: long-term results. Radiology. 1992;184:833-839.

17. Vroegindeweij $D$, Vos LD, Tielbeek AV, Buth $\mathrm{J}$, van den Bosch HC. Balloon angioplasty combined with primary stenting versus balloon angioplasty alone in femoropopliteal obstructions: a comparative randomized study. Cardiovasc Intervent Radiol. 1997;20:420425.

18. Laird JR. Limitations of percutaneous transluminal angioplasty and stenting for the treatment of disease of the superficial femoral and popliteal arteries. J Endovasc Ther. 2006;13(suppl 2):॥30-II40.

19. Schlager O, Dick P, Sabeti S, Amighi J, Mlekusch W, Minar E, Schillinger M. Long-segment SFA stenting: the dark sides: in-stent restenosis, clinical deterioration, and stent fractures. J Endovasc Ther. 2005;12:676-684

20. Murphy TP, Cutlip DE, Regensteiner JG, Mohler ER, Cohen DJ, Reynolds MR et al. Supervised exercise versus primary stenting for claudication resulting from aortoiliac peripheral arterial disease: six-month outcomes from the claudication: exercise versus endoluminal (CLEVER) study. Circulation. 2012;125(1):130-9. 
21. Hamburg NM, Balady GJ. Exercise rehabilitation in peripheral artery disease: functional impact and mechanisms of benefits. Circulation. 2011;123(1):87-97.

22. Prior BM, Lloyd PG, Ren J, Li H, Yang HT, Laughlin $\mathrm{MH}$, et al. Time course of changes in collateral blood flow and isolated vessel size and gene expression after femoral artery occlusion in rats. Am J Physiol Heart Circ Physiol. 2004; 287:H2434-H2447.

23. Yang HT, Dinn RF, Terjung RL. Training increases muscle blood flow in rats with peripheral arterial insufficiency. J Appl Physiol. 1990; 69:13531359.

24. Yang HT, Ren J, Laughlin MH, Terjung RL. Prior exercise training produces NO-dependent increases in collateral blood flow after acute arterial occlusion. Am J Physiol Heart Circ Physiol. 2002; 282:H301-H310.

25. Yang HT, Prior BM, Lloyd PG, Taylor JC, Li Z, Laughlin $M H$, et al. Training-induced vascular adaptations to ischemic muscle. J Physiol Pharmacol. 2008; 59 Suppl 7:57-70.

26. Lloyd PG, Yang HT, Terjung RL. Arteriogenesis and angiogenesis in rat ischemic hindlimb: role of nitric oxide. Am J Physiol Heart Circ Physiol. 2001 ; 281 :H2528-H2538.

27. Yang HT, Ogilvie RW, Terjung RL. Training increases collateral-dependent muscle blood flow in aged rats. Am J Physiol. 1995; 268:H1174-H1180.

28. Yu J, deMuinck ED, Zhuang $Z$, Drinane $M$, Kauser K, Rubanyi GM, et al. Endothelial nitric oxide synthase is critical for ischemic remodeling, mural cell recruitment, and blood flow reserve. Proc Natl Acad Sci U S A. 2005; 102:10999-11004

29. Watson L, Ellis B, Leng GC. Exercise for intermittent claudication. Cochrane Database Syst Rev. 2008 CD000990

30. Gokce N, Vita JA, Bader DS, Sherman DL, Hunter LM, Holbrook M, et al. Effect of exercise on upper and lower extremity endothelial function in patients with coronary artery disease. Am J Cardiol. 2002; 90:124-127.

31. Vita JA, Treasure CB, Yeung AC, Vekshtein $\mathrm{VI}$, Fantasia GM, Fish RD, et al. Patients with evidence of coronary endothelial dysfunction as assessed by acetylcholine infusion demonstrate marked increase in sensitivity to constrictor effects of catecholamines. Circulation. 1992; 85:1390-1397.
32. Gordon JB, Ganz P, Nabel EG, Fish RD, Zebede J, Mudge $\mathrm{GH}$, et al. Atherosclerosis influences the vasomotor response of epicardial coronary arteries to exercise. J Clin Invest. 1989; 83:1946-1952.

33. Brendle DC, Joseph $\sqcup$, Corretti MC, Gardner AW, Katzel LI. Effects of exercise rehabilitation on endothelial reactivity in older patients with peripheral arterial disease. Am J Cardiol. 2001 ; 87:324-329.

34. Hambrecht $R$, Adams V, Erbs $S$, Linke A, Krankel N, Shu $Y$, et al. Regular physical activity improves endothelial function in patients with coronary artery disease by increasing phosphorylation of endothelial nitric oxide synthase. Circulation. 2003; 107:3152-3158

35. Gokce N, Keaney JF Jr, Menzoian JO, Watkins $M$, Hunter L, Duffy SJ, et al. Risk stratification for postoperative cardiovascular events via noninvasive assessment of endothelial function. Circulation. 2002; 105:1567-1572.

36. Brevetti G, Silvestro A, Schiano V, Chiariello $M$. Endothelial dysfunction and cardiovascular risk prediction in peripheral arterial disease: Additive value of flow-mediated dilation to ankle-brachial pressure index. Circulation. 2003; 108:2093-2098.

37. Hiatt WR, Wolfel EE, Regensteiner JG, Brass EP. Skeletal muscle carnitine metabolism in patients with unilateral peripheral arterial disease. J Appl Physiol. 1992; 73:346-353.

38. Baver TA, Brass EP, Hiatt WR. Impaired muscle oxygen use at onset of exercise in peripheral arterial disease. J Vasc Surg. 2004; 40:488493.

39. Baver TA, Brass EP, Barstow TJ, Hiatt WR. Skeletal muscle StO2 kinetics are slowed during low work rate calf exercise in peripheral arterial disease. Eur J Appl Physiol. 2007; 100:143151

40. Hiatt WR, Regensteiner JG, Hargarten ME, Wolfel EE, Brass EP. Benefit of exercise conditioning for patients with peripheral arterial disease. Circulation. 1990; 81:602-609.

41. Huang AL, Vita JA. Effects of systemic inflammation on endothelium-dependent vasodilation. Trends Cardiovasc Med. 2006; 16:15-20.

42. Tisi PV, Shearman CP. Biochemical and inflammatory changes in the exercising claudicant. Vasc Med. 1998; 3:189-198. 
43. Turton EP, Coughlin PA, Kester RC, Scott DJ. Exercise training reduces the acute inflammatory response associated with claudication. Eur J Vasc Endovasc Surg. 2002; 23:309-316.

44. American College of Sports Medicine (ACSM). ACSM'S Metabolic Calculations Handbook. Lippincott Williams \& Wilkins, 2006.

45. Nordanstig J, Taft C, Hensäter $M$, Perlander A, Osterberg K, Jivegård L. Improved quality of life after 1 year with an invasive versus a noninvasive treatment strategy in claudicants: one-year results of the Invasive Revascularization or Not in Intermittent Claudication (IRONIC) Trial. Circulation. 2014;130(12):939-47

46. Leeper NJ, Myers J, Zhou M, Nead KT, Syed A, Kojima $Y$, et al. Exercise capacity is the strongest predictor of mortality in patients with peripheral arterial disease. J Vasc Surg 2013;57(3):72833.

47. Gardner AW, Montgomery PS, Parker DE. Physical activity is a predictor of allcause mortality in patients with intermittent claudication. J Vasc Surg 2008;47(1):117-22.

48. Garg PK, Liu K, Tian L, Guralnik JM, Ferrucci L, Criqui $M H$, et al. Physical activity during daily life and functional decline in peripheral arterial disease. Circulation 2009;119(2):251-60.

49. Campbell SM, Braspenning J, Hutchinson A, Marshall MN. Research methods used in developing and applying quality indicators in primary care. BMJ. 2003;326(7393):816-9

50. Wollersheim $H$, Hermens $R$, Hulscher $M$, Braspenning J, Ouwens $M$, Schouten J, et al. Clinical indicators: development and applications. Neth J Med. 2007;65(1):15-22.

51. KNGF Richtliin Claudicatio Intermittens. The Royal Dutch Society for Physical Therapy. www. fysionet.nl, 2003
52. Mazari FA, Khan JA, Carradice D, Samuel $N$, Abdul Rahman $M N$, Gulati $S$, et al. Randomized clinical trial of percutaneous transluminal angioplasty, supervised exercise and combined treatment for intermittent claudication due to femoropopliteal disease. $\mathrm{Br}$ J Surg. 2012;99(1):39-48

53. Murphy TP, Cutlip DE, Regensteiner JG, Mohler ER, Cohen DJ, Reynolds MR et al. Supervised exercise versus primary stenting for claudication resulting from aortoiliac peripheral arterial disease: six-month outcomes from the claudication: exercise versus endoluminal (CLEVER)study. Circulation. 2012;125(1):130-9.

54. Kruidenier LM, Nicolai SP, Rouwet EV, Peters RJ, Prins MH, Teijink JA. Additional supervised exercise therapy after a percutaneous vascular intervention for peripheral arterial disease: a randomized clinical trial. J Vasc Interv Radiol. $2011 ; 22(7): 961-8$.

55. Greenhalgh RM, Belch JJ, Brown LC, Gaines PA, Gao L, Reise JA, et al. The adjuvant benefit of angioplasty in patients with mild to moderate intermittent claudication (MIMIC) managed by supervised exercise, smoking cessation advice and best medical therapy: results from two randomised trials for stenotic femoropopliteal and aortoiliac arterial disease. Eur J Vasc Endovasc Surg. 2008;36(6):680-8.

56. Bo E, Hisdal J, Crancarova M, Stranden E, Jorgensen JJ, Sandbaek G, et al. Twelvemonths follow-up of supervised exercise after percutaneous transluminal angioplasty for intermittent claudication: a randomised clinical trial. Int J Environ Res Public Health. 2013;11(11):5998-6014 

CHAPTER 10

SUMMARY

SAMENVATTING

VALORISATIE 



\section{SUMMARY OF THE THESIS}

The aim of this thesis was to describe methods for improving the conservative management of intermittent claudication (IC) in the Netherlands. To achieve this objective, several studies were conducted to elucidate all possible indications for supervised exercise therapy (SET), to introduce a possible future outcome measure and to describe how a stepped care conservative management of IC can be implemented in a chronic-care model for the general Dutch population. In this chapter we summarize the main findings of the studies.

Management of IC requires a multidisciplinary approach. Chapter 2 provides an upto-date overview of the current literature regarding cardiovascular risk management and SET, both of which are integrated parts of the treatment for patients with IC. The development of IC is accelerated by the same cardiovascular risk factors as known for other expressions of atherosclerotic disease (i.e. coronary heart disease and cerebrovascular disease). ${ }^{1}$ Treatment of IC should therefore consist of a multicomponent therapy of cardiovascular risk modification (1), including lifestyle coaching (2) and symptomatic treatment (3). The first two components aim to prevent cardiovascular events (myocardial infarction, stroke) and related morbidity and mortality. The current evidence supports the use of SET as the primary symptomatic treatment for IC. SET improves maximum walking distance and health-related quality of life and constitutes a marginal risk of co-morbidity or mortality. This is also illustrated by the inclusion of SET in contemporary international guidelines. ${ }^{2,3}$

Although SET is considered the primary treatment for IC, it seems underutilized in clinical practice, possibly due to a limited availability of physical or exercise therapists with specific knowledge on peripheral arterial disease or SET treatment protocol. Another explanation could be differences in the views on the indications for SET, such as concomitant significant cardiopulmonary comorbidity, patient's age or aortoiliac artery obstruction as cause of IC. Chapter 3 documents current opinions of vascular surgeons and fellows in vascular surgery on SET as treatment option for IC. We specifically wanted to find out to what extent and on which criteria Dutch vascular surgeons decide to offer or deny SET to IC patients. We showed that the respondents would refer $75 \%$ of newly diagnosed patients with IC for SET. The criteria not to refer for SET were mostly based on the existence of major co-morbidity (e.g. pulmonary and cardiac complaints) or a significant iliac stenosis causing IC. Apparently, the respondents assumed that SET would not be successful in these particular patients. This could be explained by a concern for major complications or the belief that SET is ineffective in improving 
walking ability or quality of life in these particular patients. Since the arguments for not referring for SET seemed to be outdated, we concluded that SET should receive more emphasis in clinical practice. Furthermore, vascular surgeons agreed that lifestyle management should ideally be integrated in IC treatment.

Chapter 4 describes the results of a systematic review on different modes of (supervised) exercise therapy. In literature, SET usually consists of treadmill walking. ${ }^{3}$ However, alternative modes of exercise therapy (e.g. cycling, strength training) have been described, with beneficial effects on walking capacity and quality of life..$^{4-6}$ Therefore, the following question remained: Which exercise mode provides the most beneficial results? We demonstrated that there are few studies comparing alternative modes of exercise training to the standard of supervised walking exercise. The review authors identified five studies which randomised a total of 135 participants. The alternative modes of exercise therapy included cycling, strength training, and upper-arm ergometry. Although limited data are available, we showed that alternative modes of exercise therapy seem to yield results that are similar to those of supervised walking therapy. Therefore, these alternative modes may be considered useful if supervised walking exercise is not an option for the patient. However, more randomised controlled trials are needed to make a meaningful comparison between the different modes of exercise therapy.

In Chapter 5 we hypothesized that IC patients had a reduced physical activity (PA) level compared to healthy adults. A reduced exercise capacity and PA level are both strong predictors of long-term mortality. In contrast, higher PA levels are associated with less functional decline..$^{7-10}$ Weekly PA of more than light-intensity improves 5-year survival rate significantly more than light-intensity PA or no PA. ${ }^{8}$ For this reason, one could argue that the PA level is a valid outcome parameter in evaluating treatment effects of any intervention for IC. To objectively determine the PA level of IC patients and healthy adults we performed a prospective observational study using the newest generation of tri-axial accelerometers. We showed that more than half of the patients with IC did not meet the minimum recommendations for PA and public health. Compared with healthy adults, they had a significantly lower daily PA level. PA differences were due to an IC subgroup with severe functional impairments. Because a low PA level is considered a strong predictor of mortality and functional decline, all health-care workers should actively encourage PA in IC patients. 
In Chapter 6 we assessed the effect of a SET program on daily PA levels and walking behavior. We hypothesized that a SET-induced increased walking distance would improve PA levels in IC patients. Although SET was proven to be effective in improving walking distances, one can question whether an increased walking capacity is of benefit during the patient's daily life activities. In other words, if an IC patient is enabled to walk further, this does not necessarily imply that PA levels and/or exercise behavior are positively influenced. We demonstrated that a 3-month SET program increases the number of patients achieving the ACSM/AHA public health minimum recommendations for PA. The general focus of increasing walking capacity alone seems ancillary to other elements of supervision. Exercise behavior and PA might be included as outcome parameter in future IC research and eventually in clinical practice.

In the Netherlands, there are several reasons why not all patients with IC are offered an evidence-based conservative treatment, including SET. In Chapter 7 we describe the problem statement of the conservative management of IC in the Netherlands and provide a design for a national integrated care network (ClaudicatioNet) for patients with IC. Based on the chronic care model as a conceptual framework, regional care networks of general practitioners, vascular surgeons, central caregivers and physical therapists should enhance the quality of care, access, continuity, efficiency and costs of the health care system. With the aid of a national database, health care professionals will be able to benchmark patient results, while ClaudicatioNet will be able to monitor quality of care by means of functional and patient reported outcome measures. With this care intervention, integrated care pathways should be improved in the coming years, resulting in optimal quality of care for all patients with IC.

Chapter 8 describes the development of quality indicators for physiotherapy management of patients with IC. Quality indicators were developed using an online survey Delphi-procedure, based on an international six-step method to develop quality indicators. Out of 83 potential quality indicators mentioned in the Dutch physiotherapy guideline on "Intermittent claudication", consensus among the experts selected five indicators: 1) The physiotherapist measures the functional claudication distance and absolute claudication distance by performing a standardized treadmill test; 2) The outcome measures are assessed at baseline, after three months, six months, twelve months and at the end of the intervention; 3) The physiotherapist administers the Walking Impairment Questionnaire (WIQ); 4) The physiotherapist measures quality of life using the Euro Quality of Life-5D; 5) The physiotherapist checks whether patients comply with the prescribed use of medication. This set of indicators should be validated before being implemented in clinical practice. 


\section{REFERENCES}

1. Criqui MH. Systemic atherosclerosis risk and the mandate for intervention in atherosclerotic peripheral arterial disease. Am J Cardiol $2001 ; 88: 43-7$

2. Hirsch AT, Haskal ZI, Hertzer NR, et al. ACC/AHA 2005 Practice Guidelines for the management of patients with peripheral arterial disease. Circulation 2006;1 13:e463-e654

3. Norgren L, Hiatt WR, Dormandy JA, Nehler MR, Harris KA, Fowkes FG. Inter-Society Consensus for the Management of Peripheral Arterial Disease (TASC II). J Vasc Surg 2007;45:S5-67

4. Treat-JacobsonD, Bronas UG, LeonAS. Efficacy of arm ergometry versus treadmill exercise training to improve walking distance in patients with claudication. Vasc Med 2009;14(3):203-13.

5. Sanderson B, Askew C, Stewart I, Walker P, Gibbs H, Green S. Short-term effects of cycle and treadmill training on exercise tolerance in peripheral arterial disease. J Vasc Surg 2006;44(1):119-27.

6. Hiatt WR, Wolfel EE, Meier RH, Regensteiner JG. Superiority of treadmill walking exercise versus strength training for patients with peripheral arterial disease. Implications for the mechanism of the training response. Circulation 1994;90(4): 1866-74.
7. Leeper NJ, Myers J, Zhou M, Nead KT, Syed A Kojima $Y$, et al. Exercise capacity is the strongest predictor of mortality in patients with peripheral arterial disease. J Vasc Surg 2013;57(3):72833.

8. Gardner AW, Montgomery PS, Parker DE. Physical activity is a predictor of allcause mortality in patients with intermittent claudication. J Vasc Surg 2008;47(1):117-22.

9. Garg PK, Liu K, Tian L, Guralnik JM, Ferrucci L, Criqui $\mathrm{MH}$, et al. Physical activity during daily life and functional decline in peripheral arterial disease. Circulation 2009;1 19(2):251-60.

10. McDermott MM, Liu K, Ferrucci L, Criqui $M H$ Greenland P, Guralnik JM, et al. Physical performance in peripheral arterial disease: a slower rate of decline in patients who walk more. Ann Intern Med 2006;144(1):10-20 


\section{SAMENVATTING VAN DE THESIS}

Dit proefschrift heeft als doel methoden te beschrijven om de conservatieve behandeling van claudicatio intermittens $(\mathrm{Cl})$ in Nederland te optimaliseren. Om dit doel te bereiken werden diverse wetenschappelijke studies verricht naar de mogelijke indicaties van gesuperviseerde looptherapie (GLT), een nieuwe uitkomstmaat voor perifeer arterieel vaatlijden en de beschrijving van een geïntegreerd zorgmodel voor behandeling van $\mathrm{Cl}$. In dit hoofdstuk zullen we de belangriikste resultaten van deze studies samenvatten.

De behandeling van $\mathrm{Cl}$ vraagt om een multidisciplinaire benadering. De ontwikkeling van $\mathrm{Cl}$ wordt versneld door dezelfde cardiovasculaire risicofactoren als die bekend zijn voor andere atherosclerotische vaatziekten (zoals coronaire hartziekte of het cerebrovasculair accident). ' Behandeling van $\mathrm{Cl}$ dient daarom te bestaan uit multipele componenten zoals cardiovasculair risicomanagement (1), leefstijladviezen (2) en symptomatische behandeling (3). De eerste twee componenten hebben als doel cardiovasculaire events te voorkomen (zoals een hartinfarct of beroerte), met de daarmee geassocieerde morbiditeit en mortaliteit. De derde component bestaat in de dagelijkse praktijk vit een vorm van looptherapie (waaronder GLT), een invasieve procedure (zoals een dotterbehandeling, al dan niet met stent, of bypass chirurgie) of een combinatie van beide. In hoofdstuk 2 wordt een overzicht gegeven van de wetenschappelijke literatuur betreffende cardiovasculair risicomanagement en GLT als conservatieve behandeling van $\mathrm{Cl}$. GLT verbetert de maximale loopafstand en kwaliteit van leven met een minimaal risico op comorbiditeit en mortaliteit. Om deze reden wordt GLT beschouwd als de primaire symptomatische behandeling van $\mathrm{Cl}$ en is als zodanig opgenomen in diverse internationale richtlijnen. ${ }^{2,3}$

Ondanks dat GLT beschouwd wordt als de primaire symptomatische behandeling van $\mathrm{Cl}$ lijkt deze behandeling in de klinische praktijk minder vaak voorgeschreven dan verwacht. Mogelijk wordt dit veroorzaakt door een gebrek aan fysiotherapeuten met specifieke kennis van perifeer arterieel vaatlijden en/of het GLT trainingsprotocol. Een andere verklaring kan bestaan uit een verschil in denkwijze over de indicaties van GLT, zoals het voorschrijven van GLT bij patiënten met $\mathrm{Cl}$ en significante cardiopulmonale comorbiditeit, een oudere leeftijd of een iliacale stenose als oorzaak van de klachten.

In hoofdstuk 3 van dit proefschrift worden de resultaten beschreven van een in 2011 vitgevoerde enquête, met een respons van 80 procent van de Nederlandse vaatchirurgen. De enquête onderzocht in welke mate en op welke gronden Nederlandse vaatchirurgen gebruik maakten van GLT in de klinische praktijk. Hoewel het merendeel 
van de vaatchirurgen aangaf patiënten te verwijzen voor GLT, kreeg 25 procent van de patiënten geen GLT aangeboden. GLT werd door 30 procent van de vaatchirurgen niet zinvol geacht bij patiënten met $\mathrm{Cl}$ op basis van een iliacale stenose of in combinatie met cardiopulmonale comorbiditeit. Deze argumenten blijken inmiddels achterhaald in de wetenschappelijke literatuur.

De door vaatchirurgen opgegeven 75 procent patiënten die GLT aangeboden zouden krijgen, is waarschijnlijk positief gekleurd door sociaal wenselijke antwoorden. Een analyse van declaratie data van een grote zorgverzekeraar toonde dat slechts 14 procent van de patiënten met Cl primair GLT kreeg aangeboden. Daarentegen werd in 27 procent primair gekozen voor een vasculaire interventie. De oorzaak voor deze van de richtlijn afwijkende verdeling is multifactorieel. De oorzaak ligt bij zowel patiënten, huisartsen, vaatchirurgen zorgverzekeraars als beleidsmakers.

Hoofdstuk 4 beschrijft de resultaten van een systematische review naar verschillende gesuperviseerde oefenvormen. In de literatuur bestaat GLT voornamelijk uit wandelen op een loopband. ${ }^{3}$ Echter, alternatieve oefenvormen, zoals fietsen of krachttraining, zijn beschreven met eveneens positieve effecten op de loopcapaciteit en kwaliteit van leven. ${ }^{4-6}$ De vraag die wij ons stelde luidde: Welke oefenvorm leidt tot de meest gunstige behandelresultaten? In dit hoofdstuk werden 5 studies beschreven die in totaal 135 deelnemers met $\mathrm{Cl}$ randomiseerden. De alternatieve oefenvormen die beschreven werden zijn fietsen, krachttraining en arm-ergometrie. Ondanks een beperkt aantal studies, kon geconcludeerd worden dat alternatieve oefenvormen nagenoeg vergelijkbare resultaten lieten zien voor wat toename van loopcapaciteit, in vergelijking met de standaard GLT behandeling. Concluderend blijken alternatieve oefenvormen dan ook een goed alternatief voor GLT indien lopen als trainingsvorm niet mogelijk blijkt.

In hoofdstuk 5 werd de hypothese opgeworpen dat patiënten met $\mathrm{Cl}$ een verminderde fysieke activiteit (FA) hebben in vergelijking met gezonde volwassenen. Een verminderde oefencapaciteit en FA zijn beide sterke voorspellers van mortaliteit en functionele achteruitgang. ${ }^{7-10}$ Wekelijkse FA van gemiddelde of hoge intensiteit, verbetert de 5-jaars overlevingskans significant in vergelijking met geen FA of FA van lichte intensiteit. 8 Daarom kan beargumenteerd worden dat de FA een valide uitkomstmaat kan zijn om de behandelresultaten van interventies voor $\mathrm{Cl}$ te evalueren. In dit hoofdstuk werd een prospectieve observationele studie verricht om de FA van zowel patiënten met $\mathrm{Cl}$ als gezonde volwassenen te bepalen middels tri-axiale accelerometrie. Uiteindelijk bleek dat meer dan de helft van de patiënten met $\mathrm{Cl}$ de minimum ACSM aanbevelingen 
voor FA niet haalden. In vergelijking met gezonde volwassenen hadden patiënten met IC een lagere FA. Deze verschillen bleken voornamelijk verklaard te worden door de subgroep van $\mathrm{Cl}$ patiënten met meer functionele belemmeringen.

In hoofdstuk 6 werd het effect van 3 maanden GLT op de dagelijkse FA geanalyseerd. Onze hypothese was dat een toename in maximale en pijnvrije loopafstand door GLT zou leiden tot een toegenomen FA. Hoewel GLT effectief is in het vergroten van de maximale en pijnvrije loopafstand is het de vraag of deze toegenomen loopafstand van meerwaarde is voor de dagelijkse fysieke activiteit. Met andere woorden, als een patiënt met $\mathrm{Cl}$ verder kan lopen hoeft dit niet te betekenen dat zijn loopgedrag en daarmee fysieke activiteit zal toenemen. In onze studie demonstreerden wij dat na 3 maanden GLT het aantal patiënten dat voldeed aan de American College of Sports Medicine (ACSM) beweegnorm van fysieke activiteit wel degelijk significant toenam. GLT lijkt dan ook naast het verbeteren van de maximale en pijnvrije loopafstand, geschikt in het verbeteren van de dageliikse FA. FA is als potentiële nieuwe uitkomstmaat eenvoudig objectiveerbaar middels triaxiale accelerometrie en kan zodoende gebruikt worden in toekomstig wetenschappelijk onderzoek en de klinische praktijk.

Er zijn verschillende redenen waarom niet alle patiënten met $\mathrm{Cl}$ een bewezen effectieve conservatieve behandeling in de vorm van GLT kriigen aangeboden in Nederland. In hoofdstuk 7 worden de problemen rondom conservatieve behandeling van $\mathrm{Cl}$ in Nederland viteengezet. Tevens wordt de blauwdruk voor een nationaal geïntegreerd zorgnetwerk voor $\mathrm{Cl}$ beschreven (ClaudicatioNet). In dit zorgnetwerk wordt een totaalpakket van conservatieve behandeling van $\mathrm{Cl}$ aangeboden gebaseerd op een samenwerking tussen verwijzers en daartoe geschoolde fysiotherapeuten die GLT en leefstijlinterventies aanbieden. Transparantie en kwalitatief hoogwaardige zorg voor alle patiënten met $\mathrm{Cl}$ is het doel. Participerende fysiotherapeuten dienen een driejarig specialisatietraject te doorlopen. Met behulp van een online monitoring systeem wordt inzicht verkregen in de kwaliteit van de deelnemende fysiotherapeut.

Hoofdstuk 8 beschrijft het ontwikkelen van kwaliteitsindicatoren voor de fysiotherapeutische behandeling van patiënten met $\mathrm{Cl}$. De kwaliteitsindicatoren kwamen tot stand met behulp van een online vitgevoerde Delphi procedure gebaseerd op een internationale 6-stap methode voor het ontwikkelen van kwaliteitsindicatoren. Er werden 83 potentiele kwaliteitsindicatoren uit de Nederlandse richtlijn 'Claudicatio Intermittens' van de Koninklijke Nederlandse Genootschap voor Fysiotherapie geïdentificeerd. Uiteindelijk kwamen de experts tot consensus en werden 5 kwaliteitsindicatoren geselecteerd: 1) de fysiotherapeut meet de maximale en functionele loopafstand gemeten 
met een gestandaardiseerde loopbandtest; 2) de fysiotherapeut meet de functionele capaciteit middels de WIQ-vragenlijst; 3) de fysiotherapeut meet de kwaliteit van leven middels de EQ-5D vragenlijst; 4) de fysiotherapeut neemt de vitkomstmaten af bij start van de behandeling, na 3, 6, 12 maanden en bij einde van behandeling; 5) de fysiotherapeut controleert of de patiënt zijn medicatie adequaat gebruikt. Uiteindelijk zal deze set kwaliteitsindicatoren gevalideerd moeten worden voordat deze gebruikt kan worden in de klinische praktijk. 


\section{REFERENTIES}

1. Criqui MH. Systemic atherosclerosis risk and the mandate for intervention in atherosclerotic peripheral arterial disease. Am J Cardiol $2001 ; 88: 43-7$

2. Hirsch AT, Haskal ZJ, Hertzer NR, et al. ACC/AHA 2005 Practice Guidelines for the management of patients with peripheral arterial disease. Circulation 2006; 1 13:e463-e654

3. Norgren L, Hiatt WR, Dormandy JA, Nehler MR, Harris KA, Fowkes FG. Inter-Society Consensus for the Management of Peripheral Arterial Disease (TASC II). J Vasc Surg 2007;45:S5-67

4. Treat-Jacobson D, Bronas UG, LeonAS. Efficacy of arm ergometry versus treadmill exercise training to improve walking distance in patients with claudication. Vasc Med 2009;14(3):203-13.

5. Sanderson B, Askew C, Stewart I, Walker P, Gibbs H, Green S. Short-term effects of cycle and treadmill training on exercise tolerance in peripheral arterial disease. J Vasc Surg 2006;44(1):119-27.
6. Hiatt WR, Wolfel EE, Meier RH, Regensteiner JG. Superiority of treadmill walking exercise versus strength training for patients with peripheral arterial disease. Implications for the mechanism of the training response. Circulation 1994;90(4):1866-74.

7. Leeper NJ, Myers J, Zhou M, Nead KT, Syed A, Kojima $Y$, et al. Exercise capacity is the strongest predictor of mortality in patients with peripheral arterial disease. JVasc Surg 2013;57(3):728-33.

8. Gardner AW, Montgomery PS, Parker DE. Physical activity is a predictor of allcause mortality in patients with intermittent claudication. J Vasc Surg 2008;47(1):117-22.

9. Garg PK, Liu K, Tian L, Guralnik JM, Ferrucci L, Criqui $\mathrm{MH}$, et al. Physical activity during daily life and functional decline in peripheral arterial disease. Circulation 2009; 1 19(2):251-60.

10. McDermott MM, Liu K, Ferrucci L, Criqui $M H$, Greenland P, Guralnik JM, et al. Physical performance in peripheral arterial disease: a slower rate of decline in patients who walk more. Ann Intern Med 2006;144(1):10-20 



\section{VALORISATIE}

Claudicatio intermittens ( $\mathrm{Cl})$, 'etalagebenen' in de volksmond, is de eerste symptomatische viting van perifeer arterieel vaatlijden (PAV). Patiënten met etalagebenen hebben slagaderverkalking in de slagaders van en/of naar de benen. Hierdoor wordt de bloedtoevoer verminderd en ontstaat zuurstoftekort in de beenspieren. Het gevolg is dat de beenspieren tijdens inspanning sneller verzuren met krampende pijn als gevolg. Een patiënt met $\mathrm{Cl}$ kan slechts een bepaalde afstand lopen, doordat de piin toeneemt en hij viteindelijk genoodzaakt is te stoppen. Tijdens een korte rustperiode neemt de pijn geleidelijk weer af.

PAV is een veelvoorkomend probleem en heeft in Nederland een prevalentie van ongeveer 19 procent bij de populatie van 55 jaar en ouder. Rond de 50 tot 80 procent van de patiënten met PAV heeft klachten van $\mathrm{Cl}$ en zoekt hiervoor medische hulp. In de Nederlandse huisartsenpraktijk bedraagt de incidentie van $\mathrm{Cl} 2.8$ nieuw ontdekte ziektegevallen per duizend patiënten per jaar (mannen 2.7 promille, vrouwen 3.0 promille). Dit loopt op van 0.4 promille bij 25 tot 44 -jarigen tot 10.6 promille bij 75 -plussers. Gezien de actuele vergrijzing van de bevolking is de verwachting dat de incidentie en prevalentie van $\mathrm{Cl}$ verder zullen toenemen.

Het hebben van $\mathrm{Cl}$ is prognostisch ongunstig. Ruim een derde van de patiënten met $\mathrm{Cl}$ heeft tevens aandoeningen van het hart of de bloedvaten naar hersenen. Ongeveer één op de viif patiënten met $\mathrm{Cl}$ overlijdt binnen vijf jaar ten gevolge van een hartof herseninfarct. Binnen tien jaar na het begin van $\mathrm{Cl}$ krijgt 43 procent ischemische hartziekte, 21 procent een ischemisch cerebrovasculair accident (iCVA) en 24 procent hartfalen. De levensverwachting van patiënten met $\mathrm{Cl}$ is gemiddeld tien jaar korter dan die van niet aangedane personen. Daarmee is de kans op overlijden twee tot drie maal groter dan die van leeftijdsgenoten zonder PAV. Dit verhoogde risico geldt overigens ook voor mensen met asymptomatisch PAV.

Gezien de geschetste gevolgen van $\mathrm{Cl}$ behoort behandeling te bestaan uit cardiovasculair risicomanagement (1), leefstijladviezen (2) en behandeling van de symptomen (3). Zoals beschreven in de gelijknamige NHG-standaard dient cardiovasculair risicomanagement geïnitieerd te worden door de huisarts van de patiënt. Idealiter zouden ook leefstijladviezen (zoals rookstopinterventies) hier deel vit van moeten maken. Het geven van leefstijladviezen op basis van motiverende gespreksvoering kost echter tijd en vereist adequate scholing. Doordat in de Nederlands gezondheidszorg geen eisen worden gesteld ten aanzien van het geven van leefstijladviezen, worden deze hoogstwaarschijnlijk niet in alle gevallen aangeboden. 
Behandeling van symptomen van $\mathrm{Cl}$ kan zowel met een conservatieve als invasieve behandeling geïnitieerd worden. Een conservatieve behandeling, in de vorm van gesuperviseerde looptherapie (GLT), bewerkstelligt op zijn minst een vergelijkbare verbetering van de maximale loopafstand als een invasieve behandeling zoals een dotterbehandeling of bypass chirurgie. Een invasieve behandeling gaat echter wel gepaard met een verhoogd risico op morbiditeit, mortaliteit en aanzienlijke kosten. Op basis van deze basale vitgangspunten zou de behandeling van claudicatio intermittens primair moeten bestaan uit een conservatieve behandelingsstrategie. De meest recente 'National Institute for Health and Care Excellence' (NICE) richtlijn adviseert het gebruik van GLT dan ook als initiële behandeling voor alle patiënten met $\mathrm{Cl}$.

Dit proefschrift heeft als doel methoden te beschrijven om de conservatieve behandeling van $\mathrm{Cl}$ in Nederland te optimaliseren. Om dit doel te bereiken werden diverse maatschappelijke relevante wetenschappelijke studies verricht.

In hoofdstuk 3 van dit proefschrift worden de resultaten beschreven van een in 2011 vitgevoerde enquête, met een respons van 80 procent van de Nederlandse vaatchirurgen. De enquête onderzocht in welke mate en op welke gronden Nederlandse vaatchirurgen gebruik maakten van GLT in de klinische praktijk. Hoewel het merendeel van de vaatchirurgen aangaf patiënten te verwijzen voor GLT, kreeg 25 procent van de patiënten geen GLT aangeboden. GLT werd door 30 procent van de vaatchirurgen niet zinvol geacht bii patiënten met $\mathrm{Cl}$ op basis van een iliacale stenose of in combinatie met cardiopulmonale comorbiditeit. Deze argumenten blijken inmiddels achterhaald in de wetenschappelijke literatuur.

De door vaatchirurgen opgegeven 75 procent patiënten die GLT aangeboden zouden krijgen, is waarschijnlijk positief gekleurd door sociaal wenselijke antwoorden. Een analyse van declaratie data van een grote zorgverzekeraar toonde dat slechts 14 procent van de patiënten met $\mathrm{Cl}$ primair GLT kreeg aangeboden. Daarentegen werd in 27 procent primair gekozen voor een vasculaire interventie. De oorzaak voor deze van de richtlijn afwijkende verdeling is multifactorieel. De oorzaak ligt bij zowel patiënten, huisartsen, vaatchirurgen zorgverzekeraars als beleidsmakers.

Vanuit maatschappelijk perspectief zou de oplossing gevonden kunnen worden in een 'stepped care' model. Een model waarin iedere patiënt met $\mathrm{Cl}$ initieel voor drie maanden GLT krijgt. Alleen die patiënten, waarbij dit traject niet tot het gewenste resultaat heeft geleid, komen in aanmerking voor een vasculaire interventie. De verwachting is dat hiermee veel minder vasculaire interventies nodig zullen zijn bij 
deze patiëntenpopulatie. Als gevolg kan implementatie van een dergelijk 'stepped care' model dan ook leiden tot een aanzienlijke kostenbesparing voor de Nederlandse gezondheidszorg. Verdergaande kostenbesparing kan worden gerealiseerd als de diagnose $\mathrm{Cl}$ en initiatie van GLT kan worden gerealiseerd vanuit de eerste lijn. Dit kan bereikt worden door ondersteuning bij de diagnostiek naar perifeer arterieel vaatlijden (PAV) dan wel samenwerkingsafspraken met de tweede lijn en gebruikmaking van de faciliteiten van een poliklinisch vaatlaboratorium.

In dit proefschrift wordt in hoofdstuk 7 een conceptueel framework beschreven, ClaudicatioNet, waarmee landelijke dekking van regionale zorgnetwerken gecreëerd kan worden. In deze zorgnetwerken wordt een conservatieve behandeling van $\mathrm{Cl}$ geboden door samenwerking tussen verwijzers en geschoolde fysiotherapeuten die GLT en leefstijlinterventies aanbieden. Transparantie en kwalitatief hoogwaardige zorg voor alle patiënten met $\mathrm{Cl}$ is het doel. Hiertoe dienen participerende fysiotherapeuten een driejarig specialisatietraject te doorlopen. Door middel van een online monitoring systeem wordt inzicht verkregen in de kwaliteit van de deelnemende therapeut. De werkwijze van ClaudicatioNet maakt het mogelijk om ook voor andere chronische aandoeningen een 'stepped care' model te implementeren in de Nederlandse gezondheidszorg

Een andere invalshoek van dit proefschrift is het introduceren van een nieuwe vitkomstmaat voor wetenschappelijk onderzoek naar $\mathrm{Cl}$. In onderzoek naar $\mathrm{Cl}$ wordt het effect van de conservatieve of invasieve behandeling gemeten door middel van de toename in maximale, functionele of pijnvrije loopafstand. Het is echter de vraag of een toename van de loopafstand leidt tot een hogere fysieke activiteit in het dagelijks leven. Symptoomverlichting is beter meetbaar door middel van een gezondheid gerelateerde kwaliteit van leven vragenlijst, al is een dergelijke vragenlijst van voldoende kwaliteit op dit moment nog niet beschikbaar. Naast symptoomverlichting lijkt een toename van fysieke activiteit essentieel voor patiënten met $\mathrm{Cl}$, aangezien fysieke activiteit geassocieerd is met een reductie in cardiovasculaire mortaliteit en morbiditeit.

In hoofdstuk 5 en 6 wordt in twee studies beschreven hoe de fysieke activiteitvan patiënten met $\mathrm{Cl}$ in de klinische praktijk geobjectiveerd kan worden door gebruik te maken van tri-axiale accelerometrie. In de eerste studie werden 93 nieuw gediagnosticeerde patiënten met $\mathrm{Cl}$ en 36 gezonde vrijwilligers gevraagd een dergelijke accelerometer gedurende 1 week te dragen. De patiënten met $\mathrm{Cl}$ bleken een significant lagere fysieke activiteit te hebben in vergelijking met de gezonde controlegroep. Eveneens bleek dat meer dan de helft van de claudicanten niet voldeed aan de internationale 
ACSM beweegnorm voor fysieke activiteit. Wetende dat een te lage fysieke activiteit geassocieerd is met een stijging van

gezondheidsrisico, is meer aandacht voor verbetering van de fysieke activiteit bij claudicanten noodzakelijk.

In de tweede studie werd gekeken naar het effect van GLT op de fysieke activiteit bii een populatie nieuw gediagnosticeerde claudicanten. In vergelijking met het niveau van fysieke activiteit voorafgaand aan het traject GLT bleek het aantal claudicanten dat na een traject GLT wel voldeed aan de internationale ACSM beweegnorm voor fysieke activiteit toegenomen. Het lijkt dan ook aannemelijk dat GLT met incorporatie van leefstijladviezen, geïnitieerd door een daartoe getrainde fysiotherapeut, leidt tot een toename van de fysieke activiteit.

De toepasbaarheid van het meten van fysieke activiteit met accelerometrie bleek relatief makkelijk in de klinische praktijk. Dit maakt deze techniek toegankelijk voor toekomstig wetenschappelijk onderzoek. Indien we de onderzoeksresultaten willen vertalen naar de dagelijkse praktijk zou het wenselijk zijn om tot een complete set van meetinstrumenten te komen die zowel de conservatieve als invasieve behandeling van $\mathrm{Cl}$ optimaal evalueren. 
DANKWOORD 



\section{DANKWOORD}

Aan het tot stand komen van dit proefschrift hebben veel mensen bewust en onbewust een bijdrage geleverd. Enkele wil ik in het bijzonder bedanken.

Allereerst dank aan alle patiënten met perifeer arterieel vaatlijden van de vaatpoli van het Catharina Ziekenhuis die hebben bijgedragen aan dit proefschrift. Zonder uw bijdrage was dit proefschrift niet tot stand gekomen.

\section{Prof. dr. J.A.W. Teijink, beste Joep}

In juli 2009 begon ik als ANIOS in het Catharina ziekenhuis en na een enerverend zeilweekend besloot iij dat ik onderzoek bii jou ging doen. Een keuze die mij geen windeieren heeft gelegd! Jouw eindeloze energie, enthousiasme en overtuigingskracht zijn voor mij gedurende dit promotietraject van grote waarde geweest. Het is inspirerend om te zien hoe jii jouw passie met betrekking tot ClaudicatioNet weet over te brengen op mensen om je heen. Mij heb je altijd de juiste kant op gestuurd en jouw onvoorwaardelijke steun gegeven. Daarvoor ben ik je veel dank verschuldigd. Ik hoop dat ik ook in de toekomst nog veel van je mag leren op OK 7 of in een van de nieuwe hybride operatiekamers.

\section{Prof. dr. R.A. de Bie, beste Rob}

Als fysiotherapeut, bewegingswetenschapper en epidemioloog heb jii een scherpe kijk op het onderzoek naar gesuperviseerde looptherapie. Ik wil je danken voor jouw relativeringsvermogen en frisse blik. Dit heeft mij meer dan eens goede moed gegeven om vanuit een andere invalshoek naar mijn onderzoek te kijken.

\section{Dr. B.L.W. Bendermacher, beste Bianca}

Als CHIVO vaatchirurgie in het Catharina Ziekenhuis heb iij mij geleerd centraal veneuze lijnen te plaatsen: "ledere stap goed nadenken over de mogelijke gevolgen". Nog steeds als ik een lijn plaats hoor ik jouw stem in mijn hoofd en weet ik dat het goed komt. Als copromotor heb iij op eenzelfde manier bijgedragen aan de totstandkoming van dit proefschrift. Je hebt er nooit doekjes om gewonden hoe ik onderzoek moest combineren met de opleiding tot chirurg. Dankzij jouw motiverende gespreksvoering is het viteindelijk gelukt om ook dit hoofdstuk tot een succesvol einde te brengen. 
Leden van de Stichting ClaudicatioNet, beste Loes, Yvonne, Lindy, Steven, Robert, Stijn, Frans, Ben en Eva

Menig weekend reizen jullie het land door om fysiotherapeuten bij te scholen in het geven van gesuperviseerde looptherapie en leefstijlinterventies. Zelf heb ik er iedere keer weer van genoten om dit te mogen doen en deel uit te maken van het ClaudicatioNet team. Ik hoop dat we samen nog vele jaarcongressen bijwonen en viteindelijk kunnen bijdragen aan een betere kwaliteit van zorg voor alle patiënten met perifeer arterieel vaatlijden.

\section{Fysiotherapeuten ClaudicatioNet}

Jullie maken de patiënten met perifeer arterieel vaatlijden echt beter. Ik hoop dat jullie de komende jaren veel voldoening vinden in het trainen, motiveren en daarmee gezonder maken van het grote aantal claudicanten dat naar jullie verwezen gaat worden.

\section{Dr. S. Spronk, beste Sandra}

Bedankt voor de plezierige en vruchtbare samenwerking. Ik ben altijd met veel plezier naar Rotterdam gekomen en heb het fijn gevonden dat iij ons Cochrane onderzoek mede wilde begeleiden. Ik wens je veel succes met jouw huidige baan bij de Inspectie voor de Gezondheidszorg.

Dr. H.J.M. Hendriks, Prof. dr. M.G.M. Hunnink, Dr. M.J. Koelemay, Harm Gijsbers en Farzin Fakhry

Ik wil jullie hartelijk danken voor de constructieve samenwerking.

\section{Rob van Lummel en alle medewerkers McRoberts}

Dankzii jullie hulp is het gelukt om in detail de fysieke activiteit van patiënten met claudicatio intermittens te beschrijven. Jullie ondersteuning bij de analyses en het vitlezen van data was essentieel en van groot belang voor dit proefschrift. Bedankt voor jullie hulp en ik wens jullie veel succes met toekomstige innovaties.

Leden van de beoordelingscommissie, Dr. M.R.M. Scheltinga, Dr. E.M. Willigendael, Prof.dr. P.R.G. Brink, Prof.dr. M.H. Prins

Hartelijk dank voor de bereidheid dit proefschrift kritisch te beoordelen op zijn wetenschappelijke waarde. 
Dr. G.A.P. Nieuwenhuijzen en Dr. S. Nienhuijs, beste Grard en Simon

Het combineren van de opleiding Heelkunde met promotieonderzoek is regelmatig mensen teleurstellen, inclusief jezelf. Grard, als opleider zag iii al gauw dat het proefschrift zo snel mogelijk afgerond moest worden. Ik wil zowel jou als Simon van harte danken voor jullie steun en vertrouwen. Es gibts nur ein gas - Vollgas.

Maatschap Chirurgie van het Catharina Ziekenhuis, beste Marc, Philippe, Joep, Harm, Alex, Gust, Frans, Yvonne, Jean-Paul, Misha, Grard, Simon en Ignace

Wat ben ik blij om in jullie tent in opleiding te zijn. De complexiteit en volume aan ingrepen die in ' $t$ Cathrien verricht worden, in combinatie met een grote portie Brabantse gezelligheid, maakt werken in dit ziekenhuis uniek.

Fellows Chirurgie van het Catharina Ziekenhuis, beste Bart-Jeroen, Robert en Sabrina Bedankt voor alle gezellige en leerzame momenten, zowel op als buiten de OK.

Polikliniekmedewerkers Chirurgie en medewerkers vaatlaboratorium Catharina Ziekenhuis, beste Marga, Monique, Helga, Hannie, Ine, Jose, Nicole, Cindy, Dyanne, Ynette, Joyce, Nicole, Silvie, Milou, Rukiye, Arianne, Jolien, Olga, Yvonne en Anja Dank voor jullie dagelijkse inbreng in het chirurgisch bedrijf, maar zeker ook de ondersteuning bij de totstandkoming van dit proefschriff.

Onderzoekers \& semi-artsen Chirurgie Catharina Ziekenhuis

Fel zijn!

Arts-assistenten en oud-assistenten Chirurgie Catharina Ziekenhuis

Het is geweldig om deel uit te mogen maken van deze hechte club Heelkunde Helden. Ik kijk alweer met smacht vit naar het assistentenweekend, de jaarlijkse winterbijscholing, onze hardloopavonturen of de vele stapavondjes.

\section{Danielle van Dalen en Nicole Verhofstadt}

Daantje en Nicole, jullie waren vanaf het eerste moment betrokken bij het opzetten van mijn onderzoek in het Catharina Ziekenhuis. Daan, ik heb genoten van de spannende tijden waarin we samen ClaudicatioNet mee in de steigers hebben gezet. Nicole, erg bedankt voor jouw hulp bij het coördineren van mijn promotietraject. 


\section{Hugo Fokkenrood}

Perifere jongens!!! Samen met Stokkie waren wij de drie musketiers van het Chirurgie onderzoekshok. lk zal nooit vergeten hoe je als broekie tussen de haaien werd gegooid. We konden je in het begin werkelijk alles wijsmaken. Inmiddels heb je ons dubbel en dwars ingehaald, zowel qua snelheid van promoveren als qua portie assertiviteit. Ik wens de afdeling Orthopedie in het Maxima en Maastricht veel succes daarmee. Wat mij betreft blijf je altijd een 'perifere jongen' en ik kan niet wachten tot we dat dit najaar weer op onze eigen ludieke wijze mogen uitdragen tijdens de jaarlijkse ESVS trip: Porto, here we come!

\section{Rutger Stokmans}

Dat ik als 'perifere jongen' viteindelijk besluit om twee 'aorta-boys' te vragen als paranimfen voor mijn proefschrift. Het moet niet gekker worden...

Rutger, samen waren wij the Founding Fathers van het Chirurgie onderzoekshok. Hard werken, maar vooral ook veel lachen. Zo kan ik me nog een ontploffend koffiezetapparaat herinneren waar we heel Philips Nederland voor op de kop gezet hebben. Ik ben blii dat we deze zomer weer herenigd worden in het Catharina Ziekenhuis. Dat wordt ongetwijfeld weer veel lachen, zet de Prosecco maar koud.

\section{Pieter Broos}

Mijn hardcore gym buddy en vitlaatklep in barre tijden. Het laatste setje zit er op, ik heb alweer zin in een shake. Vanaf nu zal ik als jouw uitlaatklep fungeren bij het completeren van jouw proefschrift en start van je carrière als arts-assistent. De tijd is weer gekomen om locht te worden. Ben je fel?

Vrienden van $\mathrm{W} \bullet \mathrm{A} \bullet \mathrm{L} \bullet \mathrm{E} \bullet \mathrm{W} \bullet \mathrm{Y} \bullet \mathrm{N}$, beste Franken, Ter Horst, Hermsen, Van Schijndel, Van der Zande, Roerink en Van Gennip

Wat hebben we toch een mooie tijd beleefd in Nijmegen! Dankzii jullie is voor mij studeren synoniem aan de woensdagavond (gratis) bieren in Café Daen, balletje hooghouden op de bushalte van de golfbaan, zingen uit volle borst tijdens de cantus en terugfietsen van het Radboudfeest met Jan Smit. Inmiddels zijn we allemaal wat rustiger geworden. Toch hoop ik dat we nog vele avonturen mogen beleven op onze toekomstige $\mathrm{W} \cdot \mathrm{A} \bullet L \cdot E \bullet W \cdot Y \bullet N$ weekendjes.

Lieve schoonfamilie, beste Lisette, Jan, Eva en Martijn,

Dank voor jullie interesse en dat jullie altijd voor ons klaar staan, bijvoorbeeld bij onze zoveelste verhuizing naar Centerparcs Eindhoven. 


\section{Lieve Robbert en Yola}

Bro, ondanks dat we qua karakter in menig opzicht elkaars tegenpolen zijn, hebben wij het altijd super gezellig met elkaar. Veel waardering heb ik voor de manier waarop iii, samen met Yola, jullie leven ingericht hebben en het eigenlijk perfect voor elkaar hebben. Dank voor jullie jarenlange belangstelling en steun in alle aspecten van mijn leven.

\section{Lieve pap en mam}

Ik ben blij dat jullie beiden in goede gezondheid mijn promotie kunnen bijwonen. Dit proefschrift draag ik op aan jullie. De persoon die ik ben geworden is het resultaat van jullie onvoorwaardelijke liefde en steun in alles wat ik doe. Van jullie heb ik altijd de vrijheid gekregen om mijn eigen weg te kiezen. Ik zeg het misschien minder vaak als ik zou moeten maar jullie zijn werkelijk de beste ouders die een zoon zich kan wensen.

\section{Liefste Lauren}

Samen met jou kan ik de hele wereld aan

Durf ik weer mezelf te zijn

Geniet ik van elk moment

Geloof ik oprecht in elk mens

Ben ik mij bewust van mij eigen kracht

En droom ik weer over de toekomst

Lieve Lau, bedankt dat je er altijd voor me bent en mij zoveel liefde en vertrouwen geeft. Samen met jou kan ik ongelogen de hele wereld aan! 

CURRICULUM VITAE 



\section{CURRICULUM VITAE}

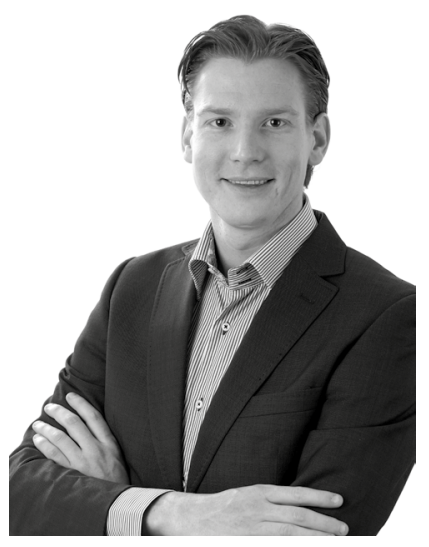
als arts-onderzoeker en coördinator ClaudicatioNet bij de sectie Vaatchirurgie in het Catharina Ziekenhuis. Gedurende deze tijd werd onder leiding van promotoren prof.dr. Teijink en prof.dr. de Bie (CAPHRI, Universiteit Maastricht) de basis gelegd van zijn promotieonderzoek naar de conservatieve behandeling van claudicatio intermittens. Op 1 juli 2012 startte hij met zijn opleiding tot algemeen chirurg en tot op heden werkt hij als AIOS Heelkunde in het Catharina Ziekenhuis te Eindhoven (opleiders dr. Nieuwenhuijzen en dr. Nienhuijs). Gert-Jan Lauret woont in Eindhoven samen met zijn vriendin Lauren Bullens. In 2016 zal hij zijn opleiding voortzetten in het Maastricht Universitair Medisch Centrum.

Gert-Jan Lauret werd geboren op 15 juli 1984 in het AZ Sint-Jan te Brugge, België. Na het behalen van het eindexamen WWO aan het Reynaert College te Hulst (Zeeuws-Vlaanderen) startte hij in 2002 met de studie Geneeskunde aan de Radboud Universiteit Nijmegen. $\mathrm{Hij}$ volgde een keuze coschap Heelkunde aan de Spitalul Clinic Judetean de Urgenta te Sibiu, Roemenië. In 2009 behaalde hij zijn artsexamen na een afsluitend coschap Plastische- en Reconstructieve Chirurgie en begon hij aansluitend als ANIOS Heelkunde in het Catharina Ziekenhuis te Eindhoven. Na anderhalf jaar klinische ervaring opgedaan te hebben vervolgde hij zijn carrière 



\section{LIST OF PUBLICATIONS}





\section{LIST OF PUBLICATIONS (PEER-REVIEWED)}

1. Hupkens P, Lauret GJ, Dubelaar I, Hartman EHM, Spauwen PHM. Prevention of wound dehiscence in palatal surgery by preoperative identification of group $A$ Streptococcus and Staphylococcus aureus. Eur J Plast Surg. 2007;29(7),321-325

2. Hupkens P, Van Loon B, Lauret GJ, Kooloos JGM, Vehof JWM, Hartman EHM, Spauwen PHM. Anteromedial thigh flaps: an anatomical study to localize and classify anteromedial thigh perforators. Microsurg. 2010;30(3),43-49

3. Yo LSF, Lauret GJ, Tielbeek AV, Teijink JAW. Effort-trombose van de vena subclavia; multidisciplinaire, acute en invasieve benadering vereist van verwijzer, vaatchirurg en interventieradioloog. Ned Tijdschr Geneesk. 2010;154:A2197

4. Lauret GJ, Teijink JAW. Ginkgo biloba heeft geen toegevoegde waarde bij de behandeling van claudicatio intermittens. Ned Tiidschr Geneesk. 2010;154:A2778

5. Lauret GJ, van Dalen HC, van Sterkenburg SM, Koelemay MJ, Zeebregts CJ, Peters $R J$, Teijink JA. When is supervised exercise therapy considered useful in peripheral arterial occlusive disease? A nationwide survey among vascular surgeons. Eur J Vasc Endovasc Surg. 2012;43(3):308-12.

6. Lauret GJ, van Dalen HCW, Willigendael EM, Hendriks HJM, de Bie RA, Spronk $S$, Teijink JAW. Supervised exercise therapy for intermittent claudication: current status and future perspectives. Vascular. 2012;Feb;20(1):12-9

7. Lauret GJ, van Dalen HCW, Hendriks HJM, Spronk S, de Bie RA, Teijink JAW. The ClaudicatioNet Concept: design of a national integrated care network for patients with intermittent claudication. Vasc Health Risk Manag. 2012;8:495-503

8. Fokkenrood HJ, Lauret GJ, Scheltinga MR, Spreeuwenberg C, de Bie RA, Teijink JA. Multidisciplinary treatment for peripheral arterial occlusive disease and the role of eHealth and mHealth. J Multidiscip Healthc. 2012;5:257-63

9. de Waure C, Lauret GJ, Ricciardi W, Ferket B, Teijink J, Spronk S, Myriam Hunink MG. Lifestyle interventions in patients with coronary heart disease: a systematic review. Am J Prev Med. 2013;45(2):207-16

10. Fokkenrood HJ, Bendermacher BL, Lauret GJ, Willigendael EM, Prins MH, Teijink JA. Supervised exercise therapy versus non-supervised exercise therapy for intermittent claudication. Cochrane Database Syst Rev. 2013;8:CD005263 
11. Mandigers L, Lauret GJ, Luyer MD, Teijink JA. Persisting pain after endovascular treatment of a symptomatic aortic aneurysm. Int J Surg Case Rep. 2013;4(9):798800

12. Lauret GJ, Fokkenrood HJ, Bendermacher BL, Scheltinga MR, Teijink JA. Physical activity monitoring in patients with intermittent claudication. Eur J Vasc Endovasc Surg. 2014;47(6):656-63

13. Fokkenrood HJ, Verhofstad N, van den Houten MM, Lauret GJ, Wittens C, Scheltinga MR, Teijink JA. Physical activity monitoring in patients with peripheral arterial disease: validation of an activity monitor. Eur J Vasc Endovasc Surg. 2014;48(2):194-200

14. Lauret GJ, Fakhry F, Fokkenrood HJ, Hunink MG, Teijink JA, Spronk S. Modes of exercise training for intermittent claudication. Cochrane Database Syst Rev. 2014;7:CD009638

15. Fokkenrood HJ, Lauret GJ, Verhofstad N, Bendermacher BL, Scheltinga MR, Teijink JA. The effect of supervised exercise therapy on physical activity and ambulatory activities in patients with intermittent claudication. Eur J Vasc Endovasc Surg. 2015;49(2):184-91 